\title{
Creating inclusive object narratives for accessible museum exhibitions
}

\author{
by
}

Carla Ayukawa

A thesis submitted to the Faculty of Graduate and Postdoctoral Affairs in partial fulfillment of the requirements for the degree of

\author{
Master of Design
}

in the

School of Industrial Design

Carleton University

Ottawa, Ontario

(C) 2021, Carla Ayukawa 


\section{ABSTRACT}

This study explores how to improve access to museum exhibits through inclusive narratives for visitors with vision loss and offers a step forward for museums concerned with inclusive research and accessible exhibition practice.

The work involved exploring how non-ocularcentric object narratives can be collected and created using an object examination exercise. The exercise draws on interdisciplinary research practices from material culture studies and participatory design, while acknowledging the distinct sensory abilities and insights of individuals with congenital and early-onset blindness. A thematic analysis was developed to inform inclusive exhibit narratives and an integrated accessible exhibition design module that was compared with an existing ocularcentric exhibit.

The broader results from the study provide insights to support multisensory and socially inclusive research in material culture studies and non-ocularcentric research practice. This experimentation with research methods supports the participation of people with non-visual mental models in curatorial research and museum exhibition development.

Keywords: Accessibility, Inclusive Design, Museology, Museum Exhibition Development, Abilitybased Design, Ocularcentrism. 


\section{ACKNOWLEDGEMENTS}

This study would not have been possible without the many individuals and institutions that gave me faith and trust in my pursuit.

I would like to begin with a deep expression of gratitude to my partner, Brian, for his love, support, and humour. His flexibility and enduring strength made my journey possible. Thank you also to our brilliant children, Mika and Nicholas, for keeping me grounded and looking toward the future.

A special thanks goes to my thesis supervisors, Prof. Chantal Trudel and Dr. Tom Everrett. Their depth of experience, vital insights, and endless encouragement permitted me to lean into the field of design research.

I would like to acknowledge and thank Ingenium-Canada's Museums of Science and Innovation; the Canadian Council of the Blind; the Natural Science and Engineering Research Council of Canada (NSERC) through its Collaborative Research and Training Experience (CREATE)'s Research and Education in Accessibility, Design, and Innovation (READi) training program; and the School of Industrial Design for their financial and collaborative support.

Thank you to my friends and colleagues who walked with me on this path and who were always so generous and timely in replying to a favour or expressing validation.

Finally, I want to thank my parents whose duty to academic research and equity compelled me to try to find another way for everyone. 


\section{TABLE OF CONTENTS}

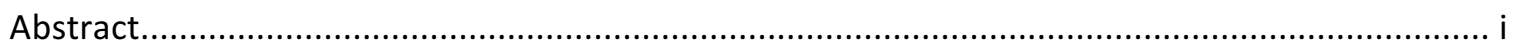

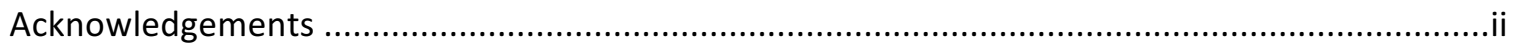

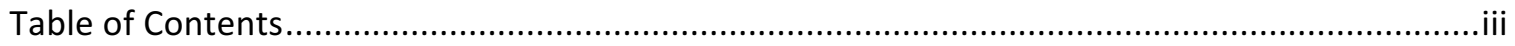

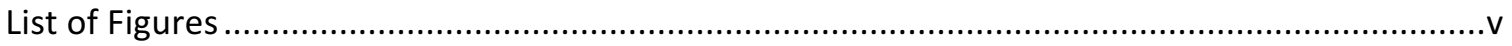

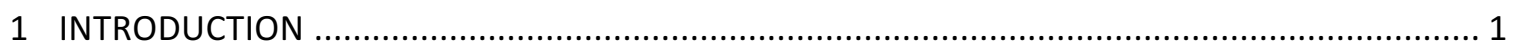

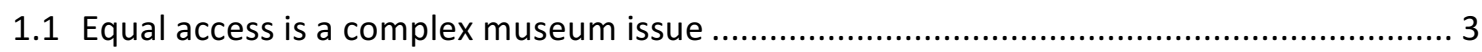

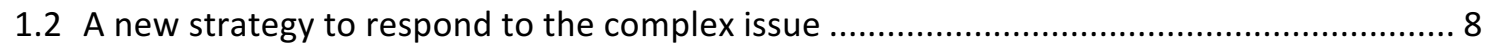

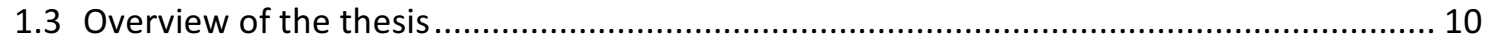

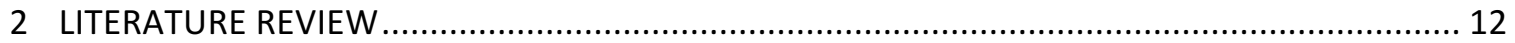

2.1 The evolution of museums and their transforming practices ......................................... 12

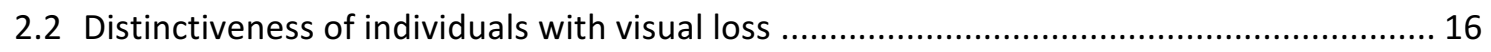

2.3 Barriers to equal access for people with visual loss .................................................... 19

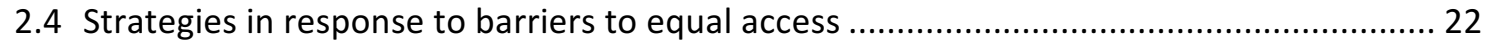

2.5 Inclusive and participatory design in exhibition development ....................................... 23

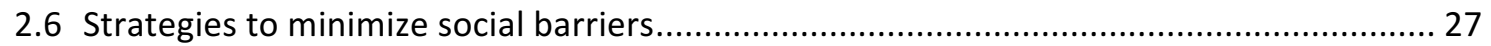

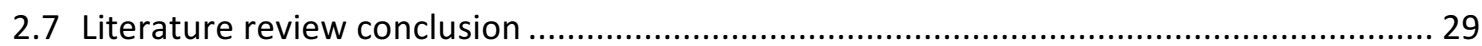

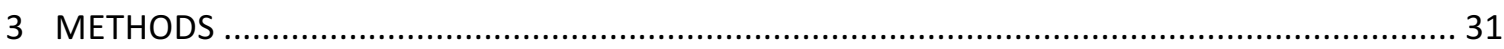

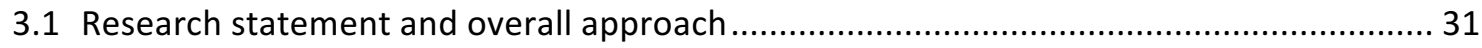

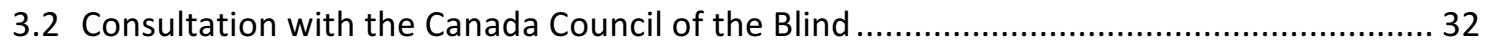

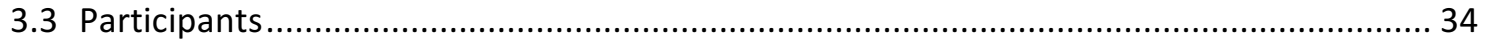

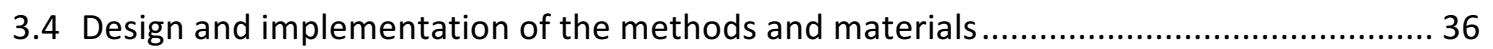

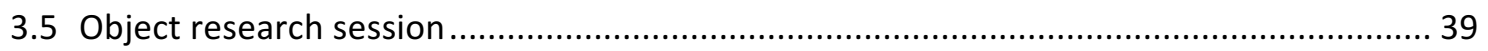

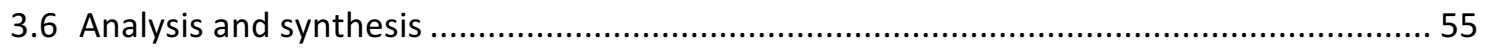




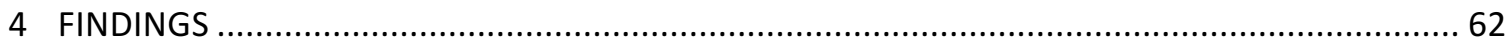

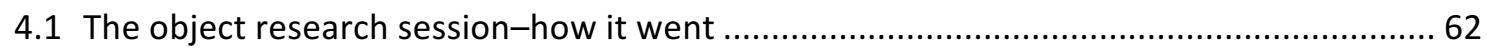

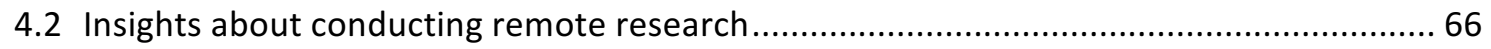

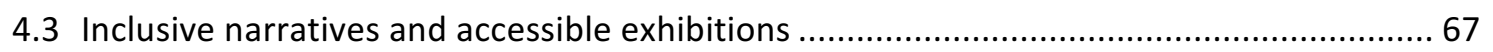

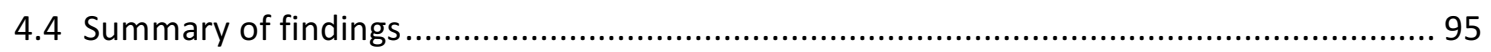

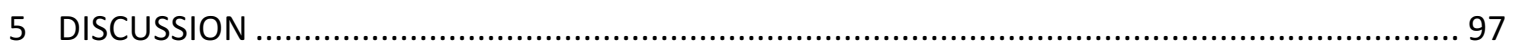

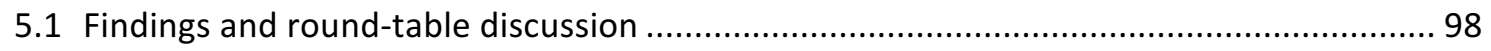

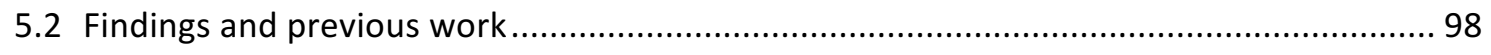

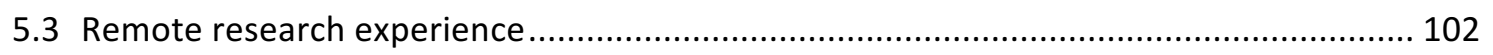

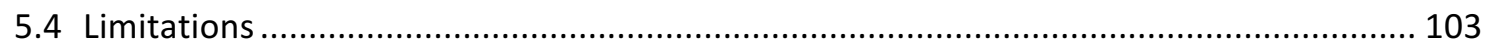

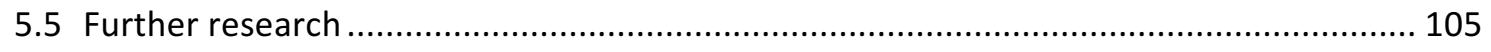

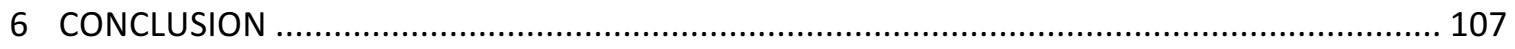

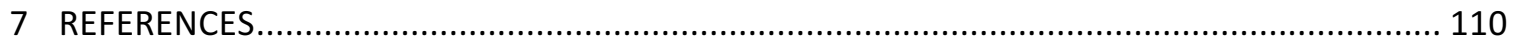

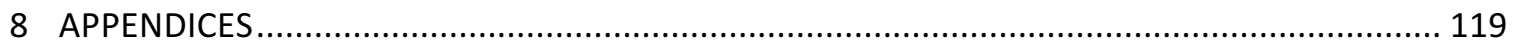

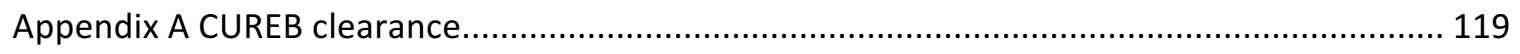

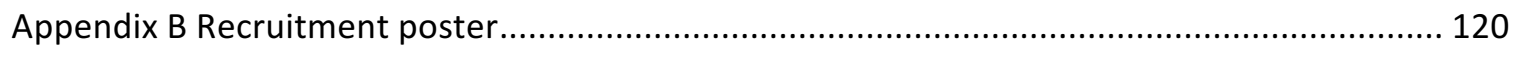

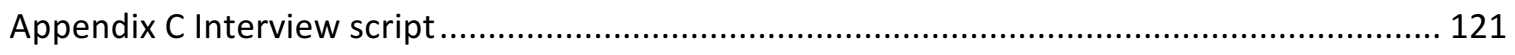

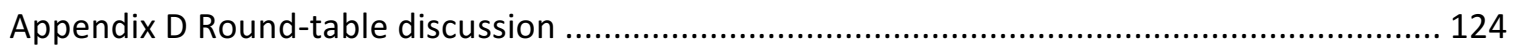




\section{LIST OF FIGURES}

Figure 1 Example of an exhibition development system 5

$\begin{array}{lll}\text { Figure } 2 & \text { Curatorial research diagram } & 6\end{array}$

$\begin{array}{lll}\text { Figure } 3 & \text { Interpretation planning diagram } & 7\end{array}$

$\begin{array}{lll}\text { Figure } 4 & \text { Exhibition design diagram } & 8\end{array}$

Figure 5 Design and implementation of methods and materials 38

Figure 6 Detail of research activities in each research phase 38

$\begin{array}{lll}\text { Figure } 7 & \text { 'DYMO' Braille labeller } & 43\end{array}$

Figure 8 Research kit labeling $\quad 44$

Figure 9 Braille labels in research kit $\quad 44$

Figure 10 'Museum-type' objects in kit $\quad 45$

Figure 11 Braille label identifies courier package as the research kit 45

Figure 12 Juicy Salif by Alessi 48

Figure 13 Contempra telephone $\quad 48$

Figure 14 After the Wall, installation view (Hunzinker, 2019) 49

Figure 15 After the Wall, exhibit detail (Stemmer, 2019) 49

Figure 16 1989-Culture and Politics, installation view (Löf, 2020) 50

Figure 17 1989-Culture and Politics, exhibit detail (National Museum Stockholm, 2019) 50

Figure 18 The Modern Eye, installation view (dominionmodern, 2013) 52

Figure 19 The Modern Eye, exhibit detail (dominionmodern, 2013) 52

Figure $20 \mathrm{Cool}$ '60s, installation view (Hunchuck et al., 2006) 53

Figure $21 \mathrm{Cool}$ '60s, showcase mural for Contempra telephone exhibit 53

Figure 22 DX Uncrated: Classic Plastics, installation view (Pesant, 2016) 54

Figure 23 DX Uncrated: Classic Plastics, exhibit detail (Pesant, 2016)

Figure 24 Schematic of visual organization of theme relationships 59

Figure 25 Visualization of analysis on MIRO $\quad 61$

Figure 26 Holding Juicy Salif two different ways 64

Figure 27 Sensory tactile detection $\quad 65$

Figure 28 Contempra telephone themes organized into exhibition topics 69

Figure 29 Visual organization of themes and inclusive exhibition topics 70

Figure 30 This study relative to previous related literature 99 


\section{Introduction}

People with vision loss are underserved in their access to museums (Chick, 2019; Vaz et al., 2020). ${ }^{1}$ To address this problem, governments have responded with equal access legislation (Committee on the Rights of Persons with Disabilities, 2014; Employment and Social Development Canada, 2021) and museums with accessibility standards (Osborne et al., 2014). ${ }^{2}$ Although some museums have been providing access through accessibility accommodations, these provisions tend to be minimal (Chick, 2019) and the possibility of an individual with vision loss being able to enjoy an independent or spontaneous visit to a museum has been characterized as inconceivable (Partington-Sollinger \& Morgan, 2011).

While the application of accessibility standards into museum practice has moved the needle forward for visitors with disabilities, equal access remains limited (Rieger \& Strickfaden, 2016; Shimamura-Willcocks, 2011). These limitations can be characterized as a mismatch between how the museums deliver equal access and what visitors with vision loss need. The causes can be attributed to a mindset where a naïve faith in accessibility standards and the complexities of the barriers to access can result in competing accommodations (Cachia, 2019; Eardley et al., 2016; Rieger \& Strickfaden, 2016) and accessibility situated within the medical model of disability (Wobbrock et al., 2011). ${ }^{3}$ This mindset can cause accessibility to be considered late in the exhibition

\footnotetext{
${ }^{1}$ The term 'people with vision loss' is used in my thesis to include people who identify as blind, partially sighted, have low vision, or no vision.

2 Accessibility Standards can be described as guidelines for developing accessible exhibitions. They were largely adapted from building standards and originally focused on improving physical access (for example heights of tables or turning radii for wheelchairs). Today they include guidance on graphic and lighting design, and multimodal content delivery (Ingenium, 2018; Smithsonian, 2012).

3 Competing accommodation: Where one group is served at the expense of another (Osborne et al., 2014);
} 
development process (Chick, 2019) and lead to exhibition designs that create or perpetuate 'othering' (Carmen, 2013; Shimamura-Willcocks, 2011). ${ }^{4}$

For years as a professional designer working in the field, I experienced a complex system where museums were tasked with producing 'accessible' exhibitions. I heard the comments that come with the pressure to balance design, content, and resources. And I knew there must be another way. In the fall of 2019, I sat in on a museum accessibility workshop to improve visitor experience. It was hosted by exhibition professionals at a national museum and included members from the Canada Council of the Blind (CCB). There was a lively discussion about the accommodations for visitors with vision loss. Within the examination of Braille, $\mathrm{QR}$ Codes, audio guides, AIRA, $3 \mathrm{D}$ printed replicas, touch-tours, and social exclusion, a member of the CCB shared a story about a museum experience with blue whales and chickadees. She recollected that ane exhibition, the topic was about the anatomy of a blue whale. Another exhibit was about diversity in bird species (with a chickadee). In both cases, she was initially excluded from the exhibition narrative because the whale was hanging from the ceiling, the birds were in showcases, and they were both outside her personal experience. She had never seen nor had a mental model of either and without the direct intervention of a competent docent, the exhibition was exclusionary. ${ }^{5}$

Her story led me to question the connection between inclusive exhibition narratives, mental models, and accessibility. How can a blue whale be awesome when you don't have a mental model of its magnitude? How can you teach that a chickadee should be treasured without a demonstration of its vulnerability? How can we create

4 Othering: "The process of magnifying differences and undervaluing similarities" (Silva \& Howe, 2012, p. 178).

5 Mental models can be described as how people understand and interpret the world based on their individual perceptions and experiences (Aehnelt et al., 2012; Norman, 2010). This term, mental model, has also been called mental imagery (Levent \& Pascual-Leone, 2014) and mental representation (Catteneo \& Vecchi, 2011; Kupers \& Ptito, 2014). 
content that is inclusive and accessible by default? Can dealing with the complexity upstream in the exhibition development process with inclusive curator research translate to better accessible design downstream?

Her museum experience was similar to stories by other members of the vision loss community who shared their frustrations with museum exhibitions made 'accessible' through standards yet gave a poor experience. Such stories demonstrate that equal access to museum services including positive visitor experience goes beyond the provision of sensory accommodations. Their stories also highlighted that museum practices that prioritize ocularcentric values and the conservation of objects (i.e., object preservation) have outranked equal access.

\subsection{Equal access is a complex museum issue}

The delivery of equal access to museum services is a complex issue as demonstrated by the members' experiences and the organization of museum disciplines in an exhibition development system. It could be described as a system generated by "the collective actions of a number of components" (Moreno \& Villalba, 2018, p 42) that can include a museum's research practices (Howes, 2014), exhibition developers' overconfidence in accessibility standards (Rieger \& Strickfaden, 2016), and competing and 'othering' accommodations to overcome a variety of barriers (Shimamura-Willcocks, 2011). If the components are not managed by the museum, the visitor bears the burden of the system's complexity that is sometimes so enormous that they do not return (Partington-Sollinger \& Morgan, 2011).

To frame the issue, Kojiro Hirose states how a key to the barriers to accessible exhibitions are institutional practices that do not sufficiently consider inclusive design at the start of the exhibition development process (Hirose, 2013). Complementary to his 
statement, Kat Holmes writes that inclusive design is a method of which accessibility is an attribute (Holmes, 2018).

Another way to interpret the issue is to compare accessible exhibition development to Tesler's law of the conservation of complexity. ${ }^{6}$ In this case, the complexity of accessible exhibitions can be borne by the different components or 'disciplines' within the museum exhibition system: the curator, the interpretive planner, the designer, or the user/museum visitor. For example, at some museums, the interpretive planners and designers tackle the issue by using accessibility standards. However, because the standards are limited in their depth of reach (and can cause competing accommodations), the visitor is then tasked to bear the remaining complexity of trying to engage with an inaccessible exhibition.

To address this issue, recognizing the barriers and developing new strategies are needed to manage the complexity and deliver equal access to museums for visitors with vision loss where inclusivity and accessibility are front-of-mind when developing exhibitions. A review of the literature identified three categories of barriers-sensory, experiential, and social-and various strategies that attempt to respond to the barriers. These strategies primarily included participatory design methods in programming and design with the vision loss community. The most current research strategies for accessible museum exhibitions for visitors with vision loss have looked beyond the practice of using accessibility standards as a response to the barriers and have identified a lack of participatory methods in curatorial research (Chick, 2019; Laurentino et al., 2021).

${ }^{6}$ Tesler's law dictates that the complexity of a system is constant and by simplifying one part of a system, the complexity is not removed but rather, shifted to another part (Norman, 2010). This law is commonly considered in the field of user experience (UX) design where application developers consider who will experience the complexity-the end user or the software designer (Moreno \& Villalba, 2018). 
Figures 1-4 illustrate the relationship between the existing curatorial research and the exhibition development system. The figures identify the different museum disciplines and how equal access through accessibility has been approached. While curatorial research is an essential part of exhibition development, its role in managing the complexity of equal access and integrating inclusive practices is not extensively discussed in the literature to date, and for this reason, perhaps unclear.

Figure 1

Example of an exhibition development system

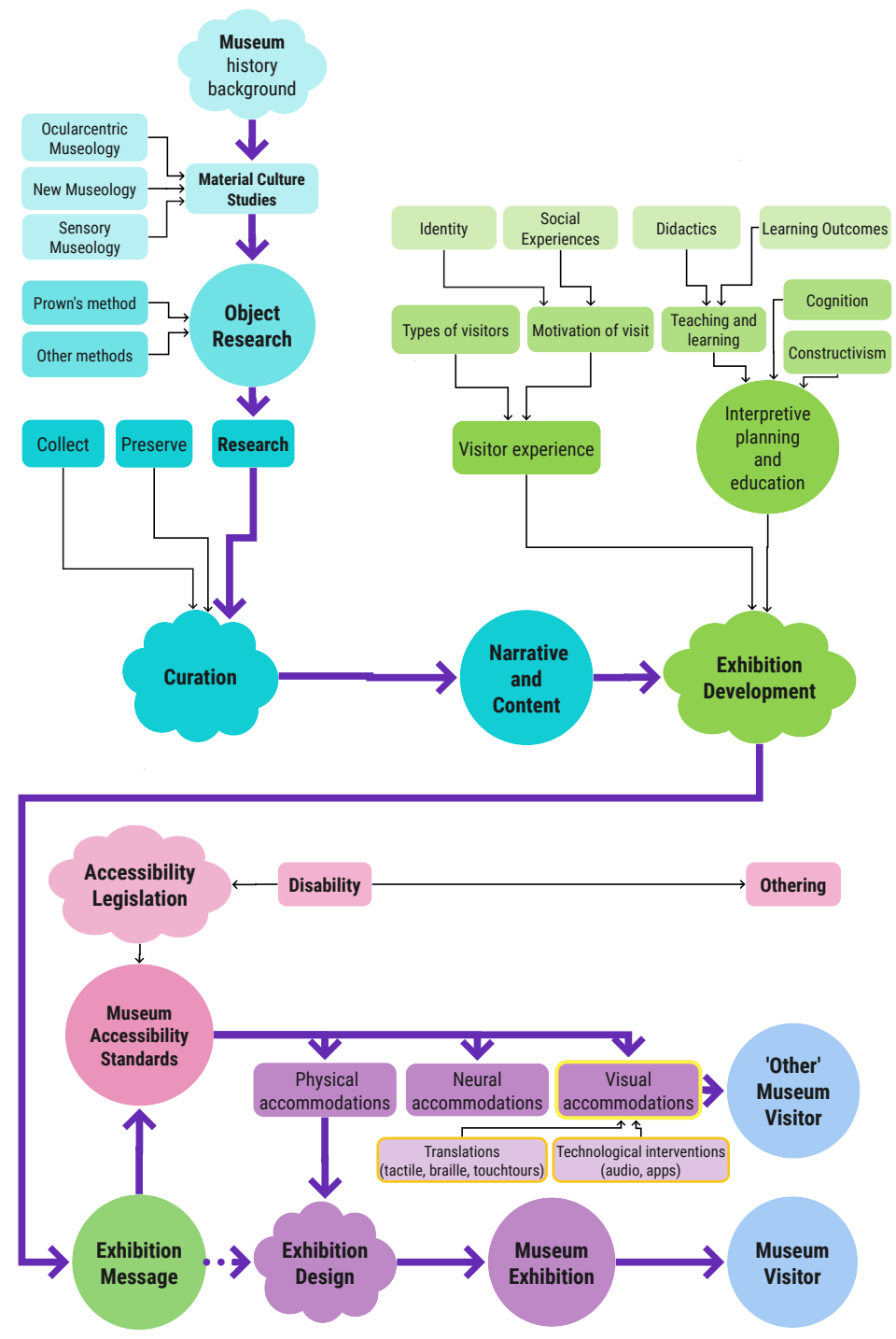

Note: This figure illustrates an overview of the exhibition development system. Figures 2 to 4 provide details of each discipline's process. 
Figure 2

Curatorial research diagram

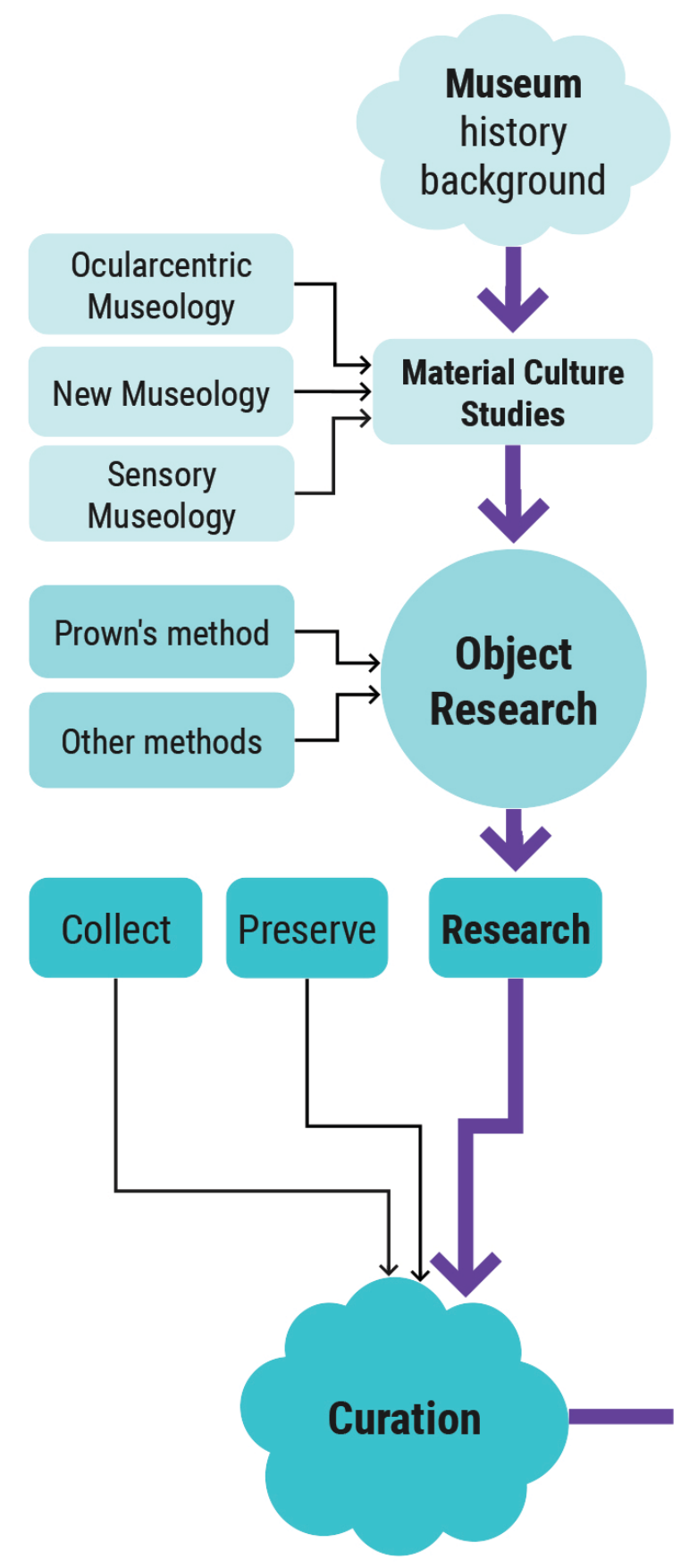

Note: This diagram provides a general schematic of curatorial research in exhibition development. The purple line follows a path that begins with the museum's mandate (seen here as History/Background). The line continues through areas of knowledge that include Material Culture Studies and Object Research. These feed into and play a role in the curator's responsibility to research. 
Figure 3

Interpretation planning diagram

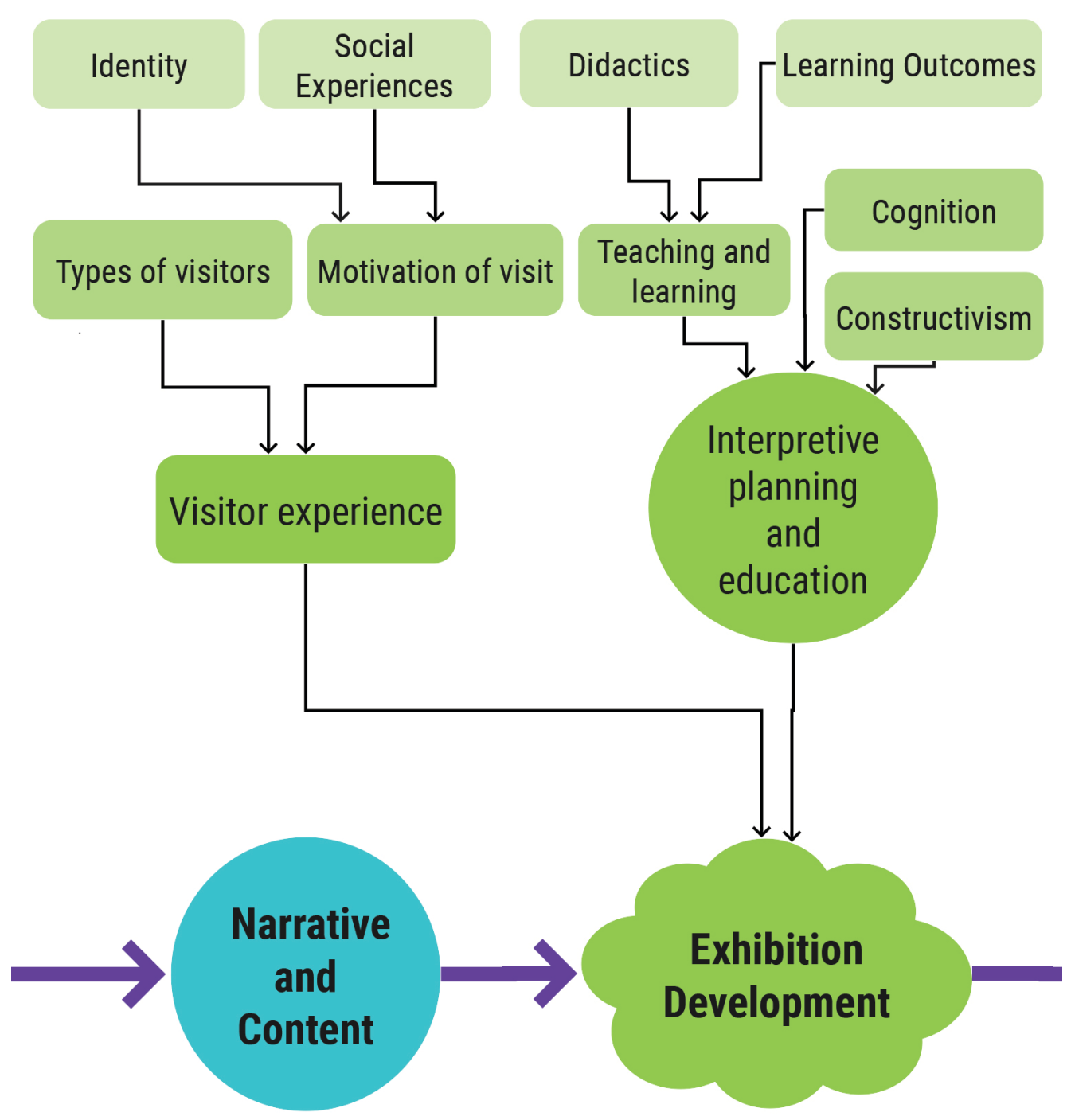

Note: This diagram provides a general schematic of exhibition development for interpretive planners. Shown are some of the influences that play a role in the responsibilities of an interpretive planner in developing exhibitions. Also shown is the contribution of curators to the Exhibition Development process that includes exhibition Narrative and Content. 
Figure 4

Exhibition design diagram

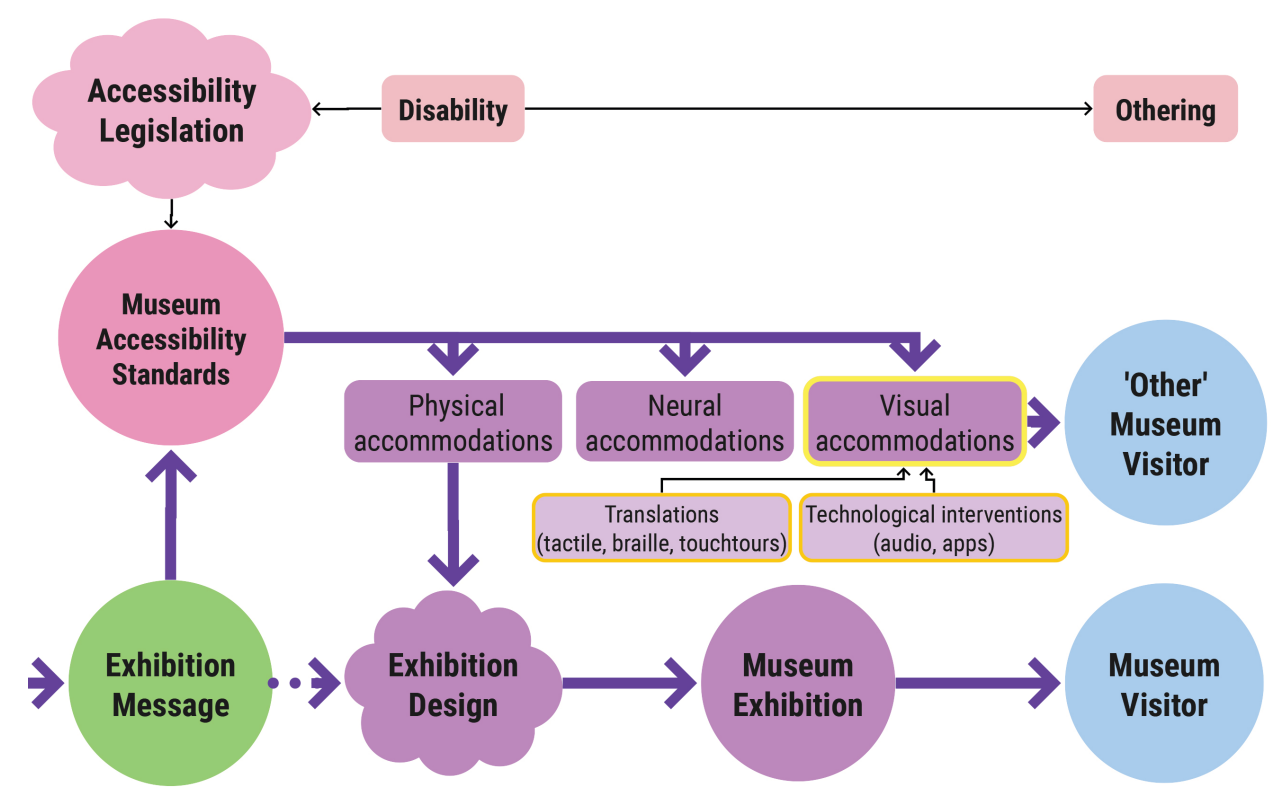

Note: This diagram provides a general schematic of exhibition design. The process begins with the Exhibition Message that is a product of the Narrative and Content from the curators and the Exhibition Development from the interpretive planners. It shows how the Exhibition Message takes a variety of paths as Museum Accessibility Standards 'add' accommodations to meet compliance to various accessibility barriers. The yellow borders related to Visual accommodations highlight my research's area of inquiry. As shown, depending on the accommodation, the outcome can be a regular museum visitor or an 'othered' museum visitor.

\subsection{A new strategy to respond to the complex issue}

The strategy explored in this study makes use of the experiences and perspectives of people with no visual memory to create inclusive exhibition narratives. Whereas accessibility for exhibitions is commonly considered downstream in the development process, this study looks at how applying inclusive practices upstream, at the outset of exhibition development-during curatorial research-can contribute to accessible exhibitions with fewer sensory, experiential, and social barriers. Objects are used in this study due to their central role in curatorial research as a means to understand a society through an object's cultural value (Pearce, 1994) and as a vehicle of inquiry or 'cultural probe', a method common in design research (Sanders \& Stappers, 2014). 
This approach to museum exhibition development draws from different disciplinary practices as noted above. But the process is centered on consultation with people who have little visual memory of objects, and therefore, distinct sensory, cognitive, and lived experiences with objects. The study combines interdisciplinary techniques derived from material culture studies (object research/readings), qualitative research methods from the social sciences and humanities (interviews in a small focus group setting), and participatory design techniques (the development of methods with the $\mathrm{CCB}$, the exploration of cultural probes with participants, the exploration of an inclusive exhibit module derived participants with participants). Data were collected from 12 participants through six 2-person interviews in focus group settings and analyzed for prominent themes using in vivo coding methods. In vivo coding was chosen since the coding process aims to stay close, and therefore, honour the voice of participants. It also aims to closely tie the interpretations and themes that emerged through the thematic analysis to these stakeholders (Saldaña, 2016).

Through qualitative research methods and working with people with vision loss, this study explores equal access and accessibility using inclusive design practices in object research. It offers an alternative strategy to accessible exhibition development by using interdisciplinary and participatory design methods that combine techniques derived from material culture studies, object research, and design research using cultural probes. Lastly, it demonstrates that collaboration with people with vision loss can further the field of sensory museology by generating non-ocularcentric insights and novel interpretations of museum objects. ${ }^{7}$

7 Sensory museology expands on traditional notions of object research by taking a multisensory perspective to examining objects. Here, the inquiry into objects goes beyond the traditional visual examination and includes sensory exploration such as sound, taste, touch, and operation (Howes, 2014). 


\subsection{Overview of the thesis}

This thesis begins with background about museum-related research, exhibition development, and the distinctiveness of people with vision loss to gain an understanding of the accessible exhibition development environment.

Through a literature review, the thesis provides an overview of accessible exhibitions by categorizing and defining the barriers faced by visitors with vision loss and the responses by museums as they try to manage the system's complexity to deliver equal access. In reviewing examples of how museums have created and responded to barriers, the question emerges 'How might we improve access through the exploration of inclusive narratives?'

The study's development is then explained with a review of the methodology that bridges design research practices with curatorial studies in material culture, a rationale for the choice of qualitative methods, the planning and design of the object research sessions, and the consultation process with the CCB. I then discuss how the different components chosen for the data collection were key to exploring non-ocularcentric interpretations of museum objects including the rationale in the choice of objects for research-Alessi's Juicy Salif and the Contempra telephone-which are often interpreted according to dominant narratives that rely on a culture of vision. I also describe how the data collection was adapted to meet the COVID-19 research ethics protocols and the requirements of participants with vision loss to participate under these unique conditions. The detailed description of the research kit's development will assist others who want to collaborate remotely with the vision loss community. The dominant curatorial descriptions and narratives of the research objects are described prior to the findings of this study but will come to the forefront again when they are compared to the inclusive narratives presented in the findings section. 
The findings from the research are presented as inclusive themes/exhibition topics and were also synthesized into a proposed exhibit module to provide an early prototype or framework for an inclusive exhibition. In the discussion section, I review the findings with reference to previous literature and touch on the reactions by the participants to the exhibit module in relation to an inclusive exhibition experience. I also discuss the limitations of the study in this section and areas of need for future work.

This strategy combines museum research practices, participatory design methods, and ability-based inclusion with people with vision loss. It seeks to demonstrate how an interdisciplinary approach to an accessible exhibition system coupled with people's voices that have been marginalized in and by design, can perhaps manage complexity, offer new insights in object research, and improve visitors' experiences in museum exhibitions.

Overall, this study focuses on consultation with this one group of people to find a response to barriers to equal access caused by ocularcentric bias and improve access through inclusive narratives. The outcome of this research also offers museum practitioners an example of how working with a group of individuals with specific sensory abilities can contribute to finding and developing inclusive exhibition topics that offer alternative reference points and common ground to support exhibition messages. 


\section{$2 \quad$ Literature Review}

\subsection{The evolution of museums and their transforming practices}

Museums are institutions with the responsibility to collect, preserve, generate, and share knowledge in the service of society (International Council of Museums, 2007). These functions can be performed in part through the research of ethnographic traditions, natural sciences, and material culture. Their values have transformed and continue to transform together with the changes in society and their needs (Abt, 2007; Papadimitriou, N., Plati, M., Markou, E., \& Catapoti, D., 2016; Saunders, 2014).

As part of its service to society, museums' values in research and exhibition transform with social, political, and learning conditions. Museums reposition themselves in how or what they focus on and their exhibitions re-interpret the stories they present for public education through this changing lens (Bennet, 1995). These museum practices transform as conditions such as social reform, decolonization, and nationalism impact society.

\subsubsection{The transformation of museum practices with society}

To appreciate their transformations, it is important to understand the origins of museums and their practices. Some of the first accounts of organized museums have been attributed to those belonging to Greek scholars' 'mouseions'-sites dedicated to the contiguity of scholarly knowledge and its materials (Abt, 2007). Later, the Roman Empire's expansion and building program led to objects being collected and displayed not only for the pursuit of knowledge but also as "trophies of conquest" (2007, p. 117). The tradition of object collections elevating their owner's standing continued through the Middle Ages. An example of a personal collection, organized into a museum and described as a cabinet of curiosities, is the Museum Calceolarium. Its owner, Francesco Calzolari, a $16^{\text {th }}$ century pharmacist, presented his objects through touch, smell, sight, 
and taste (Lorenc et al., 2007). Elite and educated guests received a multisensory experience through private tours (Abt, 2007). In time, powerful owners, such as Francesco de' Medici, amassed large and significant collections to legitimize a dynasty.

Changes in attitudes to political and social conditions were common catalysts for the transformation of museum values (Papadimitriou et al., 2016; Saunders, 2014). One significant transformation occurred in 1793, a year after the French people claimed sovereignty through revolution. As a symbol of the nation's new republican ideals, the Louvre, no longer a royal collection, re-opened in 1793 as a public museum (Abt, 2007; Bennet, 1995). In the late 1800 s, concurrent with the colonization of the New World, British leaders initiated a 'social reform' policy whereby the government took responsibility to improve 'man's' inner life, including granting public access to museums (Bennet, 1995). This gave museums an opportunity to exhibit their exotic collections and governments a venue for a national narrative that paraded the colonial treasures (Hughes, 2015).

When museums opened to the public, their practices transformed. Due to the increase in admissions to museums, multisensory interaction with collections became a liability to museum objects and, therefore, forbidden (Candlin, 2008b; Classen \& Howes, 2006; Howes, 2014). In the mid-nineteenth century, "soberly, mannerly looking" (Krmpotich, 2020, p. 96) was promoted as proper behaviour in museums and touch was associated with "vulgarity," "primitives, and children" (Candlin, 2008a, p. 283). Ocularcentric values in academia emerged to privilege the visual senses and the published word over other knowledge traditions (for example oral traditions) (Candlin, 2008b). These changes contributed to museum values transforming into ocularcentric institutions (Classen \& Howes, 2006). 


\subsubsection{Inclusion in society and its effect on museum values}

More recently, museum values have begun to shift once again as society moves toward inclusion (French, 2019; Morphy, 2015; Shelton, 2007). Institutions have been re-examining their colonial and ocularcentric values as they strive to decolonize, embrace New Museology in their curatorial practices, and involve source communities (for example, Indigenous traditions and knowledge) (Canadian Museum of History, 2020; Harrison, 1994). ${ }^{8}$ In their efforts to become less vision-based, anthropologists and historians have been re-exploring sensory research practices coined 'sensory museology' (Howes et al., 2018; Peers \& Krmpotich, 2014; M. Smith, 2007). The recent integration of (Howes et al., 2018; Krmpotich, 2020; Wood, 2019) soundscapes in museum exhibitions, and research into data reinterpreting quantitative data into audio representations-data sonification-acknowledges how the institutions are striving to become less ocularcentric (Everrett, 2019; Fogle-Hatch, 2021; Wood, 2019).

\subsubsection{The right to equal access}

The argument for expanding access to include equal access for disability finds support in this march toward public access as governments recognize how museums can play a role in shaping a national narrative and in the inclusion of marginalized communities. In 2006, the United Nations released a convention stating that persons with disabilities have a right to equal access (Committee on the Rights of Persons with Disabilities, 2014). Canada followed suit in the form of the Accessible Canada Act in 2019. The Act provides a framework for delivering equal access regardless of a person's

\footnotetext{
8 New Museology focuses on re-examining collections and object interpretation using the knowledge, perspectives and values of the originating cultures (e.g., Indigenous cultures). It is a result of more participatory curatorial practices and inclusive exhibition development.

9 In efforts to respond to calls for inclusion, collaboration and representation of Indigenous communities within museums and the Truth and Reconciliation Commission of Canada's Call to Action \#67, the Canadian Museum Association is spearheading the Reconciliation Program and the Canadian Museum of History includes curatorial research as part of their Framework for Indigenous Relations (Canadian Museum of History, 2020).
} 
physical, sensory or cognitive ability in organizations under federal funding including museums (Employment and Social Development Canada, 2021). This new societal value contributed another component to the complexity of an exhibition development system.

Museums are generally responding to the moral and legal right for equal access by developing and using accessibility standards (Rieger \& Strickfaden, 2016). The Smithsonian and Parks Canada have been leaders in this area-some of their first guidelines were published in the early 1990s. The Canadian Museum of Human Rights (CMHR) and Ingenium are also said to be leaders through their partnerships with the community and academic institutions. ${ }^{10}$ In a 2013 press release, then CMHR's president Stuart Murray, declared that their "approach to inclusive design ... will set new Canadian and world standards for universal accessibility" (Murray, 2013, p. 1). However, unlike changes in societal values related to nationalism and decolonization where the institutions publicly share how their collection and preservation practices have transformed, the majority of museums share their response to equal access for people with disabilities in relation to sensory accommodations and their adherence to building codes (Rieger \& Strickfaden, 2016).

\subsubsection{Equal access for visitors with visual loss}

Accessibility standards for visitors with visual loss tend to focus on adapting exhibitions through sensory accommodations such as audio description, touch tours, and braille labels. The guidelines are generally directed at interpretation planners and designers and the solutions to complex issues associated with the distinct mental models of visitors with vision loss may be absent. As a consequence, exhibitions are characterized as less fulfilling by visitors with vision loss because they are deemed outside of their phenomenological experience (Chick, 2018; Laurentino et al., 2021;

\footnotetext{
${ }^{10}$ Ingenium (previously the National Museum of Science and Technology) oversees three of Canada's national museums: the Canada Science and Technology Museum, Canada Aviation and Space Museum, and Canada Agriculture and Food Museum
} 
Osborne et al., 2014). This oversight can lead to a part of society that is not served by museums because of their distinct perspectives (Mashburn, 2019; Osborne et al., 2014).

This approach by institutions in their equal access policies has become a touchpoint for museum activists in accessibility (Kudlick \& Luby, 2019). The potential to be gained from an intersection between curatorial practice and disability has been a catalyst for innovative inclusive exhibitions in art museums (Cachia, 2019; Mashburn, 2019; Papadimitriou et al., 2016). Researchers and curators are inspired by 'creative access' whereby access goes beyond the physical to include "active curatorial engagement with artists who use this notion of access as a conceptual framework in their practice" (Cachia, 2019, p. 99). An example includes photographers who identify as blind and use this creative access as a starting point for a conceptual narrative through which, their photos "point us toward an equalizing truth ... we occupy the same position ... [but] we live in interior worlds. We build inner realms from what we happen to hear, feel, see" (McCulloh, 2013, p. 1).

\subsection{Distinctiveness of individuals with visual loss}

Museums have taken the opportunity to improve access by recognizing that people with vision loss are a unique community with distinct sensory abilities and mental models. By shedding the common mindset that regards disability as an illness (Wobbrock et al., 2011) and ingrained academic research values that are ocularcentric (Classen \& Howes, 2006; Howes et al., 2018), museums are benefitting from the participation of the blind and low-vision community. Their cultural and sensory distinctiveness can reveal new insights and help inform the development of more inclusive and accessible museum exhibitions. Through a re-transformation of institutional practices away from ocularcentric values and toward inclusion, museums that embrace the concept of sensory museology may better serve society. 
Individuals with vision loss have been described as having an enhanced set of sensory inputs than sighted people, which offers distinctiveness in how they interact with and interpret the world around them (Catteneo \& Vecchi, 2011). Yet, it is common for others to frame their lack of vision as a problem that requires a corrective solution (Garland-Thomson, 2019). Wobbrock's (2011) ability-based design approach and ReyesCruz, G., Fischer, J. E., and Reeves, S.'s (2020) observations of 'mundane' practices suggest that people with vision loss should be defined through their competencies rather than disabilities. By doing so, it can open doors to new possibilities in research and the realm of accessibility (Reyes-Cruz et al., 2020; Wobbrock et al., 2011). ${ }^{11}$

In the absence or reduced capacity of vision, the brain becomes receptive and responsive to other sensory inputs (Kupers \& Ptito, 2014). This leads to alternative sensory competencies and interactions with the world compared to the sighted (Catteneo \& Vecchi, 2011). An example of the consequences of this sensorial compensation is enhanced cognitive hearing abilities that allow, for example, the comprehension of screen-read speech at four times the speed of human speech (K. Smith, 2013) or enhanced thermal detection, smelling, tactile, and listening abilities (Catteneo \& Vecchi, 2011). Together, sensorial compensation has been characterized as supranormal sensory abilities (Kupers \& Ptito, 2014).

Alternative interactions and perceptions of the world as a result of supranormal sensory abilities can lead to distinct mental models (Carmen, 2013; Catteneo \& Vecchi, 2011). In this thesis, the term mental model refers to the interpretation of physical objects or people (for example, a mental representation of a stranger on the other end of the telephone) and to conceptual spatial systems (for example, an internal representation of how a website is organized or software architecture). It is with these

\footnotetext{
${ }^{11}$ Mundane practices can be described as competencies thought to be unremarkable to people with visual loss as they conduct everyday tasks (Reyes-Cruz et al., 2020).
} 
supranormal sensory abilities and distinct mental models that individuals can offer object research perspectives and insights that may have been missed because of a person's or institution's ocularcentric values and/or vision-based research practices. Yet within distinct mental models, there may be common elements or aspects of experience, interesting connections, or points of similarity between the blind and low-vision community and the sighted. For example, as demonstrated in the research by Laurentino et al., (2021), an individual with higher tactile discrimination may be able to discern differences in Mollusks' shells. Of the many interpretations that could arise from the examinations of the different shells, one that is shared between the sighted and those with vision loss could be biodiversity. Using shell patterns thus creates a mental model for understanding the theory of evolution as an adaption to environmental change (Laurentino et al., 2021).

In their attempts to develop a better understanding of the distinct abilities and experiences of people with vision loss, some museums have begun to recognize how their practices can cause barriers to equal access for people with vision loss. However, their challenge is to not only respond to the barriers but also manage the complexity of the system to create a simplified product for the visitor. Ultimately, the goal is to have access to a museum experience that offers "impromptu visits" that are "independently accessible" and adds to the "quality of our lives" (Partington-Sollinger \& Morgan, 2011, p. 11). One of the first steps to achieving this is to recognize and understand the unique barriers that people with vision loss face through consultation; a second is to find solutions that go beyond merely reacting to these barriers to transforming the very conditions that created them. 


\subsection{Barriers to equal access for people with visual loss}

As described previously, barriers often originate from ocularcentric museum values (Candlin, 2004; Laurentino et al., 2021; Papadimitriou et al., 2016; Rieger \& Strickfaden, 2016) and the medical model mindset toward disability (Wobbrock et al., 2011). The dominantly visual practices used to research and deliver exhibitions can create products that are mismatched with the sensory abilities of visitors with vision loss. Even when using accessibility accommodations to translate visual information into accessible modes (for example, using audio description), the sensory source of the knowledge can affect the way it is interpreted and understood. Because curatorial scholarship can originate from ocularcentric knowledge and research practices (Smith, 2007), simply translating this knowledge into another mode of communication does not guarantee understanding (Papadimitriou et al., 2016) or inclusion (Asakawa et al., 2018). This mismatch can create barriers that lead to sensory, experiential, and social exclusion (Holmes, 2018) that, in turn, is associated with inequitable access to education and learning (Laurentino et al., 2021).

Barriers to museum exhibitions for people with visual loss come in different forms and affect different parts of the visitor/museum relationship (Vaz et al., 2020). Sensory barriers can be found in exhibitions that prioritize the visual senses. Experiential barriers relate to exhibition narratives that are outside of some visitors' experiences of the world around them (Abt, 2007). Social barriers manifest in exhibition design strategies that differentiate, separate, and segregate visitors with visual loss from sighted visitors. These three categories of barriers can lead to unequal access, exclusion, and a poor visitor experience. Recognizing and understanding the three categories of barriers is a first step toward finding significant and proactive responses to them.

Sensory barriers are caused by exhibition practices that privilege or assume vision (Laurentino et al., 2021; Papadimitriou et al., 2016). These practices include 
vision-only access to artifacts, didactic infographics, and affective experiences generated by using scenographic design techniques. Other practices that may create barriers include decisions in exhibition development using visual assessments (for example, artifact selection based on its visual impact or exhibition design concepts based on 2D renderings). The consequences are products with an ocularcentric bias that can result in a mismatch between visitors with visual loss and exhibitions.

Experiential barriers exclude visitors from understanding and connecting with an exhibition's content or narrative. People with congenital and early-onset blindness, for example, have mental models of the world that may not reference visual-based experiences (Laurentino et al., 2021; Partington-Sollinger \& Morgan, 2011). Experiential barriers are created when exhibition narratives are derived from primarily ocularcentric knowledge, an unfortunate aspect of a curatorial approach where they may be little consideration for inclusive practice (Chick, 2019). Examples of a mismatch between exhibition narratives and the mental models of people with congenital blindness can include teaching the theory of evolution using the hide patterns of horses or demonstrating the physics of sound and light using fireworks. ${ }^{12}$ Ocularcentric exhibition narratives often rely on the use of visual-based illustrations or interactives that demand an earlier understanding of colours, patterns, or concepts such as distances in the sky (Laurentino et al., 2021). This mismatch can also include instances where an object gains relevance only through an ocularcentric phenomenon, such as the paint, Vantablack. ${ }^{13}$

Sometimes museums attempt to address sensory barriers by adding an accommodation that compensates for a visitor's disability. This mindset is prevalent

\footnotetext{
12 To understand the relationship between biodiversity and how a horse's hide pattern evolves, one would need to know what a horse looks like and understand the concept of camouflage (Laurentino et al., 2021). To understand the concept of the physics of sound and light using fireworks, one would need an understanding of the distance from and the size of a firework when it explodes in the sky.

${ }^{13}$ Vantablack is a pigment that absorbs $99.96 \%$ of light.
} 
where some institutions equate inclusive exhibitions with design strategies or features that focus on targeted modifications for a specific disability. Without careful consideration, the accommodation can create another barrier. This new barrier, a social barrier, originates from accommodations stemming from the medical model of disability where solutions focus on the visitor's "lack of ability" (Wobbrock et al., 2011, p. 1). For example, by singling out vision loss when addressing a potential issue related to understanding an exhibition message, the concept of 'othering' may naturally, and unfortunately emerge. This can lead to segregation, whereby visitors may be forced to attend the exhibition at a prescribed date when content is offered in a more accessible format, such as by a guided tour or trained docent, or rely on an alternative device, such as a personal audio device, to engage with the content. Each can have the effect of limiting a shared social experience with friends or family members who may not want to visit the museum on one of those days or the personal audio device that causes social separation and visual segregation (Asakawa et al., 2018; Shimamura-Willcocks, 2011). Another example of an accommodation that creates a social barrier is an accessible ramp provided only at the rear of a building. Such exclusionary practices can affect the visitor's experience by separating them from their social group (vom Lehn, 2010), highlighting their disability, or worse, making their experiential integration feel like an afterthought (Shimamura-Willcocks, 2011). The consequences can range from unfulfilled visitors with a diminished social experience compared to their sighted companions or people not returning to museums at all (Handa et al., 2010; Partington-Sollinger \& Morgan, 2011).

One of the major underlying problems is that barriers to equal access to museum exhibitions tend to be dealt with at the end of the exhibition development process when adaptations and accommodations are sometimes complicated and costly to execute, particularly without a full understanding of the conditions that created the barriers. Wapner (2013) describes this outcome for visitors with vision loss as "being unable to 
experience a satisfying connection with the exhibit content means that even the most extraordinary museum will be dull and disappointing” (p. 1).

\subsection{Strategies in response to barriers to equal access}

Accessibility standards are a mainstream response by museums to demonstrate their commitment to equal access through a combination of building construction standards, ergonomic guidelines, and multimodal interactive tools (Rieger \& Strickfaden, 2016). They tend to be "general guidelines" (Ingenium, 2018, p. 6) for exhibition designers that are to be "mixed and matched and tested ... to find workable solutions" (Smithsonian, 2012, p. ii). Their use is a common part of exhibition development practice. In using these standards and guidelines, museums "maintain status as a designated museum in Canada” (Rieger \& Strickfaden, 2016, p. 5). ${ }^{14}$

However, using accessibility standards as the response to equal access can be controversial (Rieger \& Strickfaden, 2016). Some critics state that they don't offer a quantitative definition of access, are poorly enforced, or are used without a deep understanding of the disabilities they serve, for instance, vision loss. As a tool to provide equal access for visitors with vision loss, accessibility standards have often missed their mark. The outcomes of a cursory delivery of 'accessible' exhibitions have lead visitors to comment that they only receive the "crumbs of collections" (Partington-Sollinger \& Morgan, 2011, p. 12), bland audio-descriptions that does not create mental images (Vaz et al., 2020), or electronic devices that lack accessible interfaces (Asakawa et al., 2018).

Another criticism of accessibility standards is how some institutions and consultants have defaulted to them without an in-depth understanding of how or why the guidelines were created (Rieger \& Strickfaden, 2016). Some see their purpose as a

\footnotetext{
14 The museum director's use of 'designated museum' as quoted in the article may refer to his/her museum's designation as a national museum. National museums must meet certain requirements for accessibility. One of those requirements is the use of accessibility standards.
} 
contractual mechanism between a project manager and consultant to deliver an accessible exhibition. In cases such as these they have a tendency to become a checklist for accessibility, often leading to 'added-on' accommodations that are sometimes poorly executed, expensive, and viewed as compromising an exhibition design concept (Cachia, 2013; Eardley et al., 2016). Without thoughtful consideration, the results can lead to competing accommodations that can impact the experience of visitors, especially those with vision loss. For example, Braille labels are often located on a didactic panel where they 'look best' or 'don't get in the way' of the printed graphics rather than in a consistent spot for visitors to find (Kudlick \& Luby, 2019).

The drive to explore alternative strategies for equal access is in part due to a growing sense of dissatisfaction with the museum experience by visitors with vision loss (Cachia, 2013). Recently, a variety of different practices have been applied in response to these challenges including participatory design methodologies, Techné, and multisensory art. ${ }^{15}$ Such strategies tend to reimagine exhibition development practices outside the tools and techniques of accessibility standards to prevent the barriers to access rather than react to them.

\subsection{Inclusive and participatory design in exhibition development}

The following examples demonstrate how institutions and researchers have explored different strategies for accessible exhibition development in response to some of the barriers described above. These strategies focus on the inclusion of the disability community but differ in methodology and research goals. The first example describes the development of an exhibition in 2015-one of the first exhibitions that included people with disabilities on an exhibition development team. The next example explores how an

\footnotetext{
15 Techné has been defined as an intersection of co-design and ability-based designed where the designer embraces the practical knowledge of people with disabilities (Rieger et al., 2020).
} 
exhibition development team used participatory design as a means to reexamine accessibility standards with the goal of establishing a 'toolkit' for how to integrate codesign into exhibition development practices. ${ }^{16}$ Finally, the third example explains how another team of researchers took participation further by including people with vision loss in the creation of exhibition content.

The first example of inclusion for accessible exhibition development is the temporary exhibition Patient No More hosted by the Ed Roberts Center. In 2015, this exhibition, produced by the San Francisco State University, was groundbreaking in its exhibition narrative-the story of disability activism in America-and with the direct participation of people with disabilities in consultation and prototyping during the exhibition development (Kudlick \& Luby, 2019). The motivation for the researchers to include the participation of the disabled community was to highlight the "limitation of traditional museums and ... the social inequalities built into the museum model itself" (p. 60). This project, a one-off and niche topic, revealed the lack of knowledge and expertise on how to 'do' access within a participatory exhibition development environment. It also brought to light how competing accommodations are difficult to avoid and can affect visitor experience due to the variety of access needs.

The next example also incorporated inclusive and participatory design methods into the exhibition development process using design thinking and co-design as a means to create fundamental change in the mindsets of exhibition designers, and for institutional executives to "understand that designing for disabilities as a positive and exciting opportunity for innovation" (Hesseldahl et al., 2018, p. 155). This 2018 research project explored the barriers to positive accessible exhibition development experiences and the creation of tools to prevent barriers at the Wellcome Collection and the Helen

\footnotetext{
${ }^{16}$ Co-design is a participatory design method where designers and non-designers work together to develop a design (Sanders \& Stappers, 2007).
} 
Hamlyn Centre for Design through inclusive exhibition development with persons with disabilities. The intent was to change exhibition developers' perception that equal access 'limits creative freedom' and that accommodations are a list of 'deliverables'. The goal was to integrate people with disabilities into exhibition development throughout the process. The research team found that collaborating with and including people with disabilities on the exhibition development team created "empathetic links, increased awareness, and forged a direct dialogue” (p. 158). This study left the researchers hopeful that through the participation of users with disabilities, designers could feel "encouraged and inspired by access design as a space for innovation and experimentation" (p. 159).

This third example took participation and inclusion one step further upstream in the exhibition development process. The research team focused on embodied experiences and perspectives-Techné-as a starting point for the exhibition narrative and to demonstrate that someone with vision loss can give new meaning to a design (Rieger et al., 2020). In one study, children with congenital blindness were asked to photograph their environments. One child presented a series of photos that included 'close-ups' of a gym mat as a representation of good memories and "no fear when falling” (p. 9). Other photos by children are images taken in the direction of sound (one was of a radio and another of a view outside a window). The photo series offered an insight into their multisensory experiences and memories. In another study, a photographer who is blind presented his $2 \mathrm{D}$ works along with $3 \mathrm{D}$ representations with audio-description (Puxley et al., 2016). The photo exhibition explored themes of personal experience and "how we construct our worlds and ourselves" (McCulloh, 2013, p. 1). In both studies, the intent of the explorations into vision-free photographic arts was to tap into the insights and perceptions of the participants' mental models to help demonstrate that multisensory and multimodal participatory approaches can forge creative insights and 
new understandings of how they perceive the world. The results of both studies contributed to the notion of perceptual expertise ${ }^{17}$

These three studies and participatory strategies attempted to respond to barriers by developing exhibition narratives that were inclusive with respect to the mental models and sensory abilities of visitors with disabilities and visual loss, bringing multisensory modalities into the mainstream (Eardley et al., 2016). The Patient No More project illustrated the need for scholarship and experience in 'doing' access and the Wellcome Collection exhibition showed how the collaboration and participation of people with disabilities can create empathy and dialogue with exhibition developers to change the institutional attitude toward accessibility, from stifling creativity to a space for innovation and experimentation. The photographic Techné explorations demonstrated the unexpected insights and artistic value of the mental models of people with congenital blindness. The disability community contributed directly to the exhibition development beyond consultation and testing. Such work demonstrates the value of participation in educating exhibition development practitioners about the creation of art, and about the benefits of understanding and engaging with differing mental models.

Participation was a strategy to improve accessible exhibition development practices. However, within these studies, participation was contingent on pre-established curatorial narratives. The exhibition topics focused on disability and, therefore, the participation of people with disabilities was obvious. By directly tying the participatory methodology to disability, it resulted in each exhibition being focused on that particular niche topic, disability. In doing so, the projects also-albeit inadvertently or unconsciously-contributed to the concept of 'othering', a social barrier which, to some, perpetuates marginalization (Mashburn, 2019; Shimamura-Willcocks, 2011).

${ }_{17}$ Perceptual expertise can be described as how one's selective attention and unique attitude by which one perceives the world "concentrates on some features ... and ignores others" (Stolnitz, 1993, p. 9). 


\subsection{Strategies to minimize social barriers}

Minimizing social barriers can contribute to all visitors gaining equal access to museum services. One important museum service identified by museum researcher, John Falk, is the shared social experience (2009).

Through his research, Falk interpreted that one of the main motivations for visitors to attend a museum was to share an experience with friends and family. Part of a shared experience includes the spontaneous decision, multigenerational interaction with the exhibition and afterward, and memories of the experience. The museum visitors surveyed admitted that they rarely were aware of the exhibition topic when they made their decision to visit, suggesting that social considerations and potential barriers to social experience demand examination.

However, in current exhibition development practice, social barriers are not always front of mind. When exhibition development teams respond to sensory barriers, they often rely on accommodations that are sometimes only intended for individuals with vision loss, for example, audio headsets, touch tours, or special docent tours. In some cases, the headsets limit the ability to hear group conversations and special tours are only offered on certain days. This separation of individuals from their visitor group or the denial of the spontaneous visit can create a social barrier and be considered 'othering' by marginalizing an individual.

In response, some museums have explored strategies to produce inclusive exhibition narratives upstream into the curatorial phase of exhibition development. Here, a motivation for non-ocularcentric participation inspired the development of creative curatorial narratives with minimal sensory barriers at the outset. These exhibitions included topics such as biodiversity, 3D printing, and multisensory art.

A first example presents the theory of evolution and Charles Darwin's visualbased research to create an educational exhibition that is complementary to the mental 
models of people with vision loss. Laurentino et al. (2021) identified a need for equity in science education to create a more diverse scientific community since the lack of accessibility has led to few scientists with visual loss. They wanted to eliminate the barriers to understanding scientific concepts. An inclusive and accessible exhibition about the theory of evolution and biodiversity was their starting point. The challenge was to replace the commonly used examples in the visual-based research (such as a collection of Galapagos finches or the Industrial Revolution's peppered moth) with something nonvisual that provided compelling and demonstrative evidence of the theory of evolution (Laurentino et al., 2021). The curators and educators at the museum consulted with the blind community to create an inclusive exhibition and test techniques for developing non-visual scientific teaching tools that demonstrated biodiversity, the tree of life, and the theory of evolution. They used Mollusks' shells arranged according to their phylogenetic categorization of the shells' characteristics in size, shape, and texture. They were organized according to their niche environment and climate. Visitors were encouraged to touch the shells to grasp the concept of "phylogenetic patterns and common ancestry" (2021, p. 4). This study helps prove the value of collaboration between curators and people with vision loss and the significance of non-visual mental models when formulating the interpretive plan for exhibitions presenting content and concepts that are traditionally ocularcentric-based.

A research project, this one conducted by the National Centre of Craft and Design in Lincolnshire in the United Kingdom, explored a co-design strategy to actively include people with vision loss in designing an exhibition. The goal of this research group was to create an inclusive exhibition using the principles of co-production with the user, curator, and designer. Compared to the studies mentioned earlier in the thesis, the exhibition topic-3D printing-was not a niche topic related to disability and was relatable to visitors who are sighted, blind, and partially sighted. This study recognized 
that by considering inclusive design and curatorial principles in the exhibition development process, social barriers could be addressed. What was discovered was that co-creation, co-design, and co-production techniques are by tradition, predominantly visual (Chick, 2019). What is interesting in this project is how the researchers employed and struggled with Elizabeth Sanders' ocularcentric-based participatory design methods. Despite this, the study demonstrated that inclusion in the exhibition development process delivered a more accessible product with fewer accommodations that led to 'othering' and was engaging to all museum visitors.

A final example speaks to how a curatorial commitment to an inclusive narrative and to equal access led Utah Arts Alliance to create the art exhibition dreamscapes. Curators conceived of this exhibition as a completely immersive, multisensory, and accessible art show without social barriers. The exhibition presented art that was socially inclusive and spoke about universally shared experiences-dreams (Utah Arts Alliance, 2021). The multisensory engagement of the art pieces, whose curatorial concept recognized that visitors may be engaging with content through touch, sound, smell, taste etc., permitted all visitors, including those without vision or partially sighted, to experience the exhibition together, side-by-side with no social barriers.

\subsection{Literature review conclusion}

Knowing that the common practice employed by museums to respond to equal access are tools directed primarily at sensory barriers, there exists an opportunity to realize the goal of equal access and prevent marginalization with a strategy that is engaging to all and contributes to a shared social experience. The result of the strategy could ultimately offer museum visitors with vision loss an "impromptu visit" that is "independently accessible" and adds to the "quality of [their] lives" (Partington-Sollinger 
\& Morgan, 2011, p. 11). In essence, the visitor would not experience the complexity of the exhibition system and their visit would be considered interesting and worthwhile.

The literature review focused on the research and practices of interdisciplinary participation. Literature directly related to digital technologies was not included as part of the review as I wanted to concentrate on curatorial research related to inclusive narratives. While digital technologies can play a role in minimizing barriers, my study aimed to leverage the existing sensory abilities of individuals with vision loss.

The researchers presented in this literature review have explored different strategies. They have studied how the participation of a user group in the exhibition development process with the goal of improving an accessible visitor experience, has progressed from consultation and collaboration to recognizing the distinct mental models of people with vision loss, and finally, to experiencing the universal appeal of an exhibition conceived at its outset as multisensory. The literature review reveals how interdisciplinary participation (user, curator, designer, interpretive planner) is key in the development of content that complements the sensory abilities and mental models of people with vision loss and that exhibitions that minimize social barriers tend to be those that are conceived as inclusive and accessible in the curatorial concept.

Emerging from the literature review is a framework for further study that explores the idea of minimizing experiential and social barriers through participation and inclusion at the outset of exhibition development in curatorial research. These prior works point to the importance of a strategy to create an exhibition narrative that complements the mental models of people with vision loss and minimizes the potential for sensory barriers. These initiatives also suggest that societal change toward the right to equal access should parallel other societal changes and transform museum practices. 


\section{Methods}

\subsection{Research statement and overall approach}

This study is focused on exploring a new accessibility strategy to improve access to museums through inclusive narratives wherein people with distinct sensory abilities are given the opportunity to participate in the exhibition development process. Through the design of object research sessions, the aim was to collect the perspectives and insights of participants with congenital and early-onset blindness as they examine 'museum-type' objects. ${ }^{18}$ What they shared in these sessions was then analyzed and interpreted using thematic analysis to find points of interest, similarity, or difference between their object narratives and those developed by sighted individuals. The intention of the exhibit module activity was to study how these sessions, and the insights that emerged through the analysis, could potentially inform an inclusive with integrated accessibility exhibit design for an object that had traditionally been exhibited in an ocularcentric manner. The object's new inclusive narrative and its intuitively accessible presentation were evaluated in a proposal of this exhibit module for discussion with the participant group. This study was reviewed and was provided clearance from the Carleton University Research Ethics Board (CUREB) Appendix A.

This chapter outlines the design and application of the qualitative methods used in this study within the context of interdisciplinary research practice. First I describe the framework for the object research sessions, including consultation and study planning, the inclusion criteria for the participants with congenital and early-onset blindness, the criteria for the objects that were selected for examination, the design of the interview script and participant guidance, and the design of the research kit that was sent to

\footnotetext{
${ }_{18}$ In this study 'museum-type' objects are similar to objects found in museum collections. Section 3.5.4 speaks more about the choice of objects for examination in this study.
} 
participants. Next a description is provided for how the thematic analysis was conducted and how several themes/exhibition topics emerged. Following the analysis is an outline of the rationale used to select one of the themes and develop it further into an exhibit module. Lastly is a description of how I circled back and consulted with the participants in a round-table discussion about the proposed exhibit module, to discuss the application of the results that emerged from the thematic analysis and how it compares with the object's previous ocularcentric exhibit design and narrative. Through this discussion, I identify insights gained from the participant group about the inclusivity of the object narrative, the accessibility of the exhibit module and their experience as participants in the object research sessions.

\subsection{Consultation with the Canada Council of the Blind}

The planning process for the study was carried out in consultation with the Canada Council of the Blind (CCB), who made recommendations for overall wording throughout the development of written materials, assisted in the recruitment of study participants and development of the research kits, and pilot-tested the object research sessions. Wording used to describe sensory abilities followed the CCB's recommendations. For example, the word 'distinct' was suggested over 'unique', and person-first labeling was recommended (for example, using 'person with a vision loss' not 'blind person'). The CCB member noted that a person who identifies as having vision loss might choose to self-identify using vision loss, blindness, partially sighted, or low vision as terms.

Due to restrictions imposed by the COVID-19 pandemic, the CUREB protocols for distance research and the provincial stay-at-home mandate throughout the duration of this study required a different approach for conducting and recording the object research sessions than originally planned. What would have typically occurred in a dedicated 
room with audio and video camera equipment to capture dialogue and interactions with the objects transitioned to remotely recorded sessions.

The design of the remote object research sessions presented some challenges related to ethical conduct which are important to note. For example, the initial concept for the research kit suggested that each kit include a video camera that would be set up to record the session and then be returned. I would then download the recording from the device to my computer for review. The challenge was twofold: one, I would not be able to guarantee the quality of the recording; and two, the participant would most likely require an assistant to operate the video recording device. The assumption that the participant may require assistance with operating the recording device is based on the likelihood that it would be both novel to participants in terms of the interface and may require support to operate due to a common bias towards ocularcentric affordances in the designed environment (Bulk et al., 2020). Requiring an assistant, as part of the participant inclusion criteria was not recommended by the CCB. They suggested instead that members use the camera function on their smartphone or laptop, which would allow them to contribute to the study independently, without the need for an assistant.

When a prototype of the research kit was built and the logistics for recording were finalized, the object research session was pilot-tested with a member of the CCB. The pilot test included delivery and pick-up of the research kit and a performance of the interview script. The CCB made recommendations to differentiate the packing material from the research components, and to design the internal boxes that contained the objects so that they might be opened in a straightforward manner.

Although the necessity for remote research added a layer of complexity to data collection, specifically by requiring the development and dissemination of a research kit, the process contributed positively to participant recruitment and focus group pairings, which is discussed in further detail in the next section. 


\subsection{Participants}

Twelve participants with congenital and early-onset blindness were interviewed in six sessions in focus group settings within their own homes (2 people per session) over the span of three months. The data was recorded using an online video-conferencing platform (Zoom) and participants used specially designed remote research kits that contained objects that are close representative examples of objects found in museum collections.

Participants that fit the criteria for participation (congenital and early-onset blindness) were recruited to offer non-visual insights and impressions of the two 'museum-type' objects and to share any related life experiences (if applicable). Apart from these inclusion criteria, participants had to be familiar with the Zoom application, able to handle an object the size of a laptop, and to be over the age of 18 (see Appendix B for recruitment poster). ${ }^{19}$ The participants were recorded as they examined each of the objects in four stages (see Section 3.5.2 Interview and guidance scripts).

At the outset of the study, it was expected that the inclusion criteria would make participant recruitment difficult. ${ }^{20}$ However, with the support of the CCB-whose members disseminated information about the study through their communication networks-this was not the case. The CCB distributed the invitation-to-participate through email and a screen-readable PDF poster. A member also posted the email onto an electronic bulletin board, Torshout, which provided additional reach. Since the sessions were to be conducted remotely, this also allowed for recruitment across Canada and not just the immediate Ottawa area, as participants could be situated wherever courier delivery services were available and where participants had access to a stable

\footnotetext{
19 Adults 18 years and over were targeted as participants for recruitment to keep the protocol in the minimal risk category.

20 In Canada, the percentage of people who are blind (20/1000 vision) is less than $3 / 10000$ people (Maberley et al., 2006).
} 
Internet connection. Within three weeks, a pool of over twenty potential applicants had identified as being interested in participating in the study.

Participants who responded to the invitation to participate underwent two levels of screening. The first screening established their level of blindness and its onset. The second screening established if they met the remaining inclusion criteria. One participant was disqualified due to their location in the United States, since couriering the research kit across the border would have created additional complexities with scheduling and international customs. Only individuals over 40 years old who responded to the invitation to participate during the recruitment phase met the inclusion criteria.

A bit of background about each participant offered a basis for creating compatible focus group pairings. During the screening process, participants volunteered information such as their age, profession, and interests. Participants were paired based on similar interests, age, and personality traits to contribute to balanced interactions and increase the potential for mutual understanding and shared life experiences around certain objects and topics. Three participants identified as male and the remaining as female.

The participants ranged in age from 40 to 70 years of age even though the invitation-to-participate included individuals 18 years and older. This age range of participants may be related to a natural demographic shift towards the prevalence of older adults in this group who could meet the inclusion criteria of congenital and earlyonset blindness. This demographic shift is most likely due to recent advances in paediatric medicine, where the early developmental risks of retinopathy of prematurity (that result in congenital blindness) have been mitigated (Shah, 2016).

Communication with the participants was either by email or telephone. This included recruitment screening, informed consent (participants preferred oral over written consent followed with a screen-readable PDF), shipping and pickup instructions, and courier tracking numbers embedded as a link to the courier website. When possible, 
the participants gave precise courier delivery instructions that allowed them to locate the delivered package, since the couriers were using contactless delivery during the pandemic. The Zoom link was emailed 24-hours before the session with "Museum Research Session" in the subject line. A $\$ 100$ honorarium, funded by a grant from Ingenium was paid to each participant to recognize their contribution.

\subsection{Design and implementation of the methods and materials}

The qualitative research methods used to support data collection and analysis emerged from the interdisciplinary framework of the study. Techniques used in material culture studies, participatory design practice, and strategies to include the participants' perspectives and distinct knowledge were the elements that informed the research approach. The figure shown on Figure 5 illustrates the relationships between the methods, disciplines, and techniques of each research phase. Figure 6 illustrates the activities within each phase. The design of the object reading sessions was informed by consultation with the $\mathrm{CCB}$, but also through a review of semi-structured interview techniques and the use of cultural probes. Open-ended questions about 'museum-type' objects were developed with the aim of producing a variety of participant responses. The data collection was further supported by the use of cultural probes (Sanders \& Stappers, 2014) which is a common practice in design research to assist in eliciting responses or stories from participants. ${ }^{21}$ A focus group interview setting was preferred to allow participants to draw on each other's comments when formulating their insights and impressions (Sharples \& Cobb, 2015).

The data collected from these focus groups was distinct to the participant group and was thus analyzed and interpreted using thematic analysis through a manual

${ }^{21}$ Cultural probes are tools to provoke inspirational responses from a particular group who can offer data about their cultural perspectives and experiences (Gaver et al., 1999). A cultural probe can offer researchers an object that initiates a process for engagement with participants to create a dialogue and explore the object's associated social contexts (Woodward, 2016). 
process of in vivo coding to retain the participants' language and perspectives (Saldaña, 2016). From the analysis emerged a series of themes/exhibition topics. The topics were evaluated to find one that had the richest data to best answer the research question ' $\mathrm{How}$ might we improve access to museum exhibitions through the exploration of inclusive narratives?'. The topic was used to develop a creative synthesis of the results in the form of an exhibit module. At the same time, a corresponding ocularcentric exhibition was compared to the exhibit module to frame the potential differences in approach and potential for access. The new exhibit module and the ocularcentric exhibition were presented to members of the participant group with the intent to engage in discussion about the research approach and the development of inclusive and accessible exhibitions. 
Figure 5

Design and implementation of methods and materials

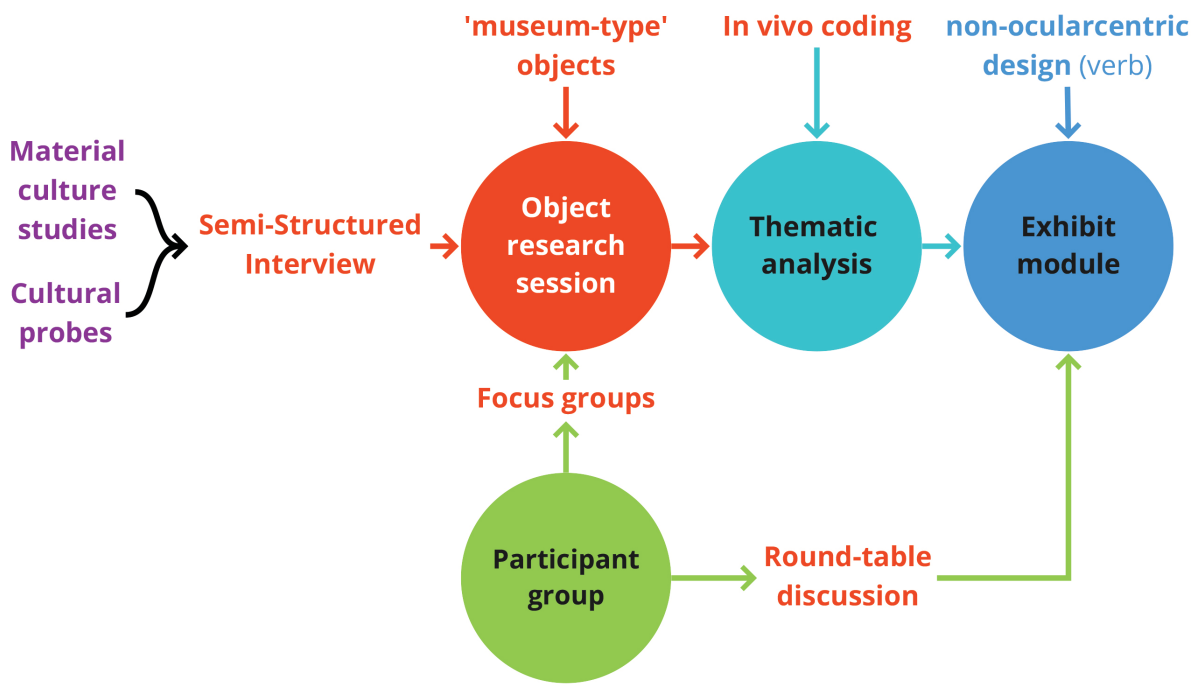

Note: This figure illustrates the relationship between the methods, disciplines, and techniques and the sequence of research phases when the methods and materials for the study are implemented (see Section 3.4). It also illustrates when collaboration with the participant group occurs via the Focus group and Round-table discussion.

Figure 6

Detail of research activities in each research phase

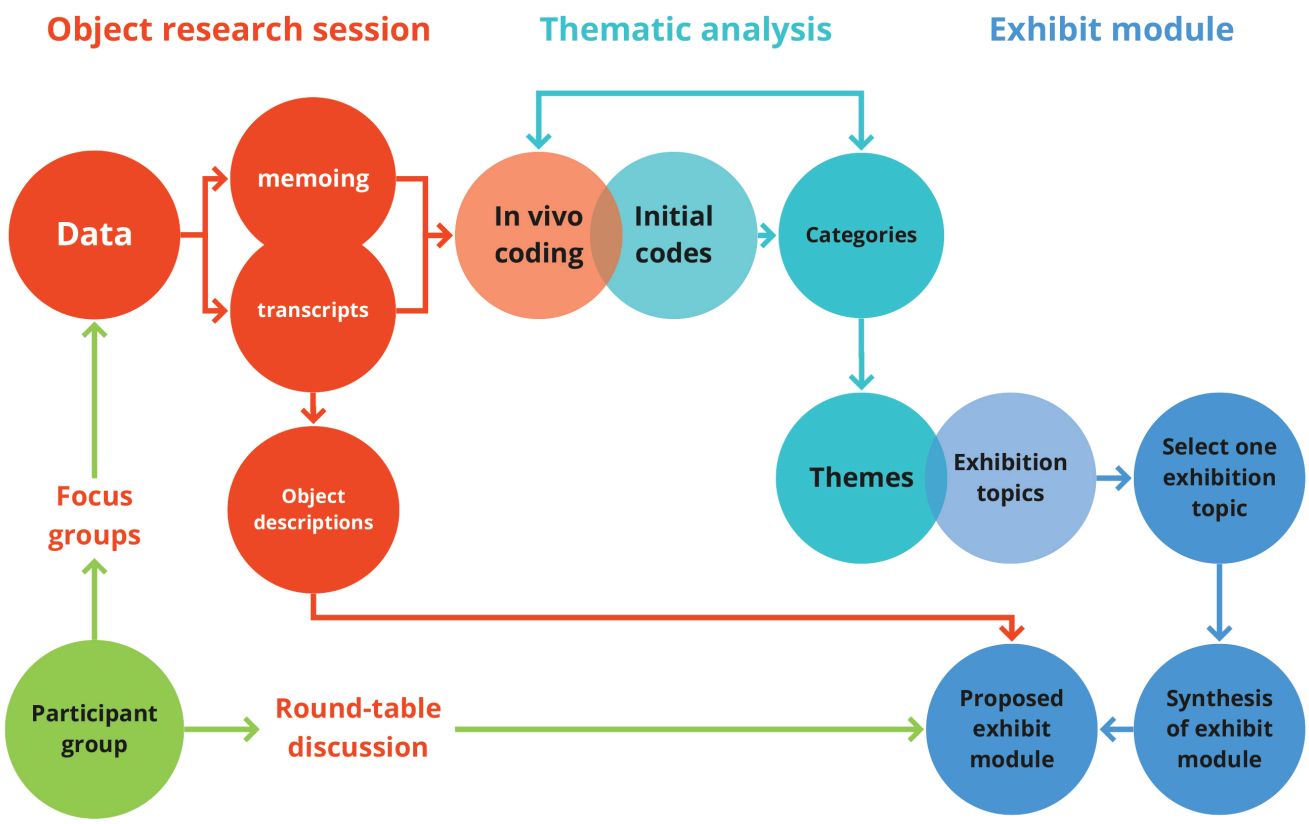

Note: This figure illustrates the process of activities within each research phase. 


\subsection{Object research session}

The object research session was central to the study's data collection. The goal for the session was to collect the insights related to 'museum-type' objects from people with no visual memory. The session was designed with an interdisciplinary framework drawing on research techniques from material culture studies, sensory museology, and participatory design research using cultural probes.

This framework was influenced by John Schlereth's system of scaffolded questions to progressively describe, understand, and interpret objects (1986) and Bill Gaver's use of cultural probes design to provoke responses from a particular group who can offer information about their cultural perspectives and experiences (1999). The inquiry took the form of semi-structured interviews within 6 small focus group settings ( 2 people per session, for a total of 12 participants). An interview script, including a sequence of open-ended probing questions, helped me to guide the session to stimulate conversation and allow participants to bring forth interpretations that were front-ofmind to their own experiences. The prompts were prefaced by phrasing such as "from your perspective..." or "based on your experiences..." to reduce the possibility that participants would feel they were being tested or that there was a correct response. Each object reading session was two hours in length, to allow for participants to thoughtfully share their perspectives and experiences. A breakdown of the sequence of events in each session is outlined below.

1. The participants received a research kit through contactless courier delivery.

2. I met the participants remotely via Zoom.

3. I assisted participants with adjusting their camera on their devices for a clear video recording of their hands, object, facial expression (if possible).

4. I performed a check-in with the participants through the following steps:

a. introductions;

b. reviewing the research kit and its contents; 
c. reviewing what to expect in the session;

d. answering questions; and

e. providing an overview on using the nitrile gloves.

5. I initiated the video and audio recording.

6. I prompted participants in the examination of the first object. ${ }^{22}$

7. Break

8. I prompted the participants in the examination of the second object.

9. The participants removed their gloves for gloveless examination.

10. I performed a check-out with the participants covering the following:

a. answering questions;

b. discussing the format for compensation;

c. discussing the repacking of the research kit with the return label;

d. discussing the courier pickup instructions; and

e. stopping the recording.

\subsubsection{The practice of wearing nitrile gloves}

One of the interdisciplinary practices that informed the design of the object research sessions was material culture study. Research in material culture is common in museums and typically involves a close, physical examination of an artifact or group of artifacts, often by hand. In this study, participants were instructed to wear nitrile gloves, as curators or researchers would in a museum context, to simulate a realistic museum experience and maintain the claim that suggests that this research project's accessibility strategy can be applied in real life. ${ }^{23}$ In museum practice, the debate about the educational value of handling and touching versus the need to protect and conserve the object is judged in favour of gloves (Howes, 2014) though with recent shifts in values toward knowledge from source communities, this practice may change (Peers \& Krmpotich, 2014). To accommodate for both possibilities, at the end of each session the

\footnotetext{
22 The order of the object examination was alternated for each focus group to reduce the chance of responses for one object to influence the comments for the next.

${ }_{23}$ Nitrile gloves protect the object from skin oils and the skin from the object (Mason, 2018).
} 
participants were invited to remove their gloves and interact with the object. This served an additional interpretive function, as most conveyed that the gloves limited their tactile competency.

\subsubsection{Interview and guidance script}

The script for the semi-structured interview sessions was organized into seven phases and five categories of questions based on the scaffolded questions in Schlereth and Prown's (1994) system of inquiry for object research and Gaver's (1999) use of cultural probes. The first category included questions asking the participants to think aloud as they examined and actively described the objects (for example, its weight, materials, surface texture). This was to establish a familiarity with the object and for the researcher to gain insights on what physical characteristics were most compelling to participants (Prown, 1994). The second type of questions continued with descriptions, but here the participants were directed to comment more subjectively on the object's design relative to their interaction and/or experience with the object (for example, How you think its weight/shape/sound contributes to its function?). At this point the dominant curatorial narrative about the object was shared and participants were asked about their impressions of the narrative. The third category of questions asked the participants to share their perspectives on the role of the object relative to their life experiences. Here, the object acted as a cultural probe to create a process of engagement and the participants were encouraged to share stories or anecdotes associated with the object or the object's social context (Sanders \& Stappers, 2014). The fourth category prompted the participant to remove their gloves and share their sensory experience. The final question asked participants to reflect on the entire research exercise.

An interview script (see Appendix C) was developed to support consistency and clarity when directing and prompting the participants in the object research sessions. The script included directions for the video set-up in Zoom, reminders of terminology, 
and check in/check out procedures. For example, when directing the participant in the video set-up through Zoom, the script included reminders to say, "move your camera ...” rather than "move your phone ..." to minimize confusion between moving the Contempra telephone, laptop, or smart phone. The script also included reminders to consult and record the format of their compensation for their participation. ${ }^{24}$

\subsubsection{Remote research kit}

The research kits were conceived of in response to the various COVID-19 protocols that necessitated remote research. For example, COVID-19 protocols outlined by the Carleton University Research Ethics Board limited in-person participant interactions in research studies. Provincial and municipal governments also mandated their own series of stay-at-home rules throughout the study timeline, and the Canadian government published directives at the time of the study related to virus transmission and the disinfection of materials. This context created unique challenges for the study design and the data collection. The solution was to create a kit containing the necessary components to allow for participants to conduct the object research at home.

With the assistance of the CCB, the research kits were designed to facilitate the needs of participants who are blind. These kits, as shown in Figures 8, 9, and 10, included the tools necessary for participants to conduct remote object research safely and independently. It contained the two 'museum-type' objects, two sizes of nitrile gloves, a pre-filled return courier label, packing material, and packing tape. The CCB lent me a 'DYMO' Braille labeler (Figure 7) to label all the components in Braille. The kit's exterior was identified with Braille, allowing participants to identify the courier package from others delivered to their homes as seen with the black Braille label (Figure 11). The envelope containing the nitrile gloves was also identified to differentiate it from the

\footnotetext{
24 The three choices of compensation (etransfer, cheque, or Amazon gift card) reflect the stay-athome mandate and the unpredictable availability of accessible online services from each participant's banking institution.
} 
packing material. Each of the interior boxes containing the objects and the roll of packing tape was also identified with Braille.

The logistics for receiving and returning the kit were designed to be contact-free and not require participants to leave their homes. The research kit was designed to withstand the rigours of intercity couriering. The size, shape, and weight were carefully considered to be cost efficient, robust, and easy to disinfect. The objects were protected by disposable bubble wrap, high-density foam, and corrugated cardboard. Each participant was couriered a research kit through FedEx except for one which I delivered to a local participant.

Figure 7

'DYMO' Braille labeller

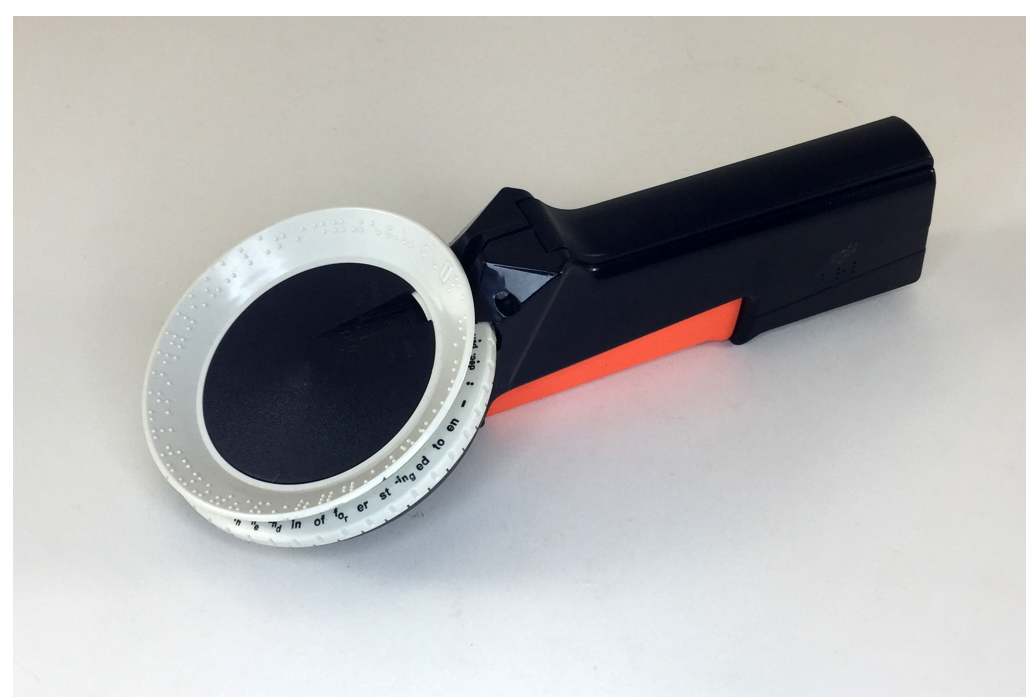

Note: The 'DYMO' Braille labeler can create custom raised dot adhesive plastic labels (available through the Braille Superstore (www.braillebookstore.com)). 
Figure 8

\section{Research kit labeling}

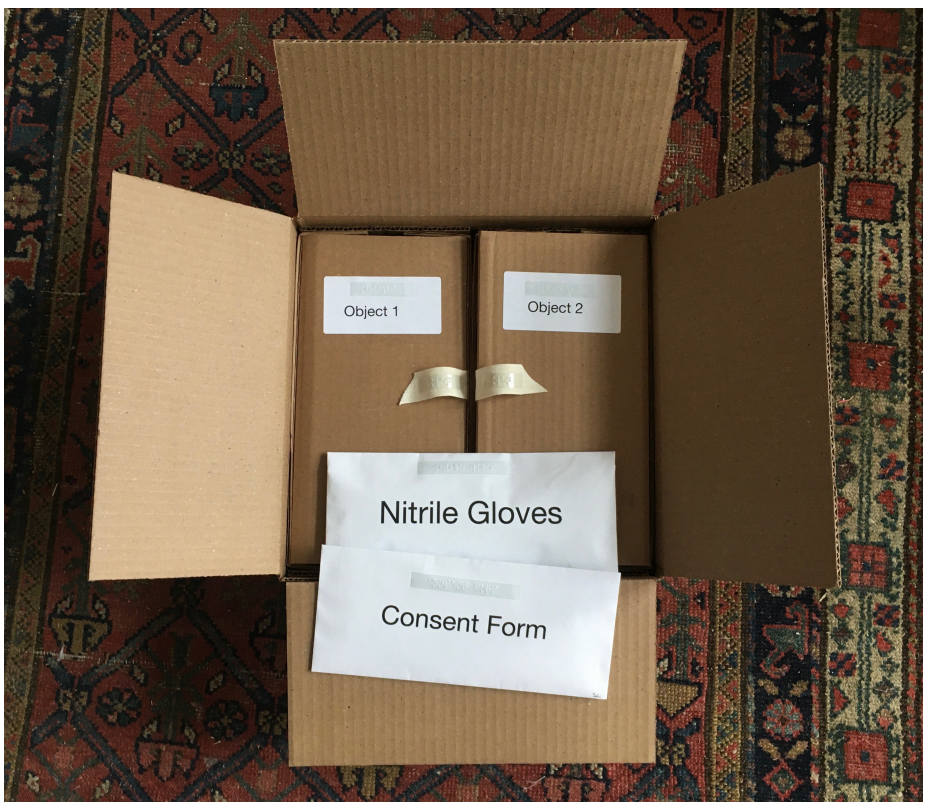

Note: This image shows how the contents of the research kit were labeled with Braille. The labels allowed the participant to identify the contents and to prevent some items from being mistaken for packing material, for example the envelop with the gloves.

Figure 9

Braille labels in research kit

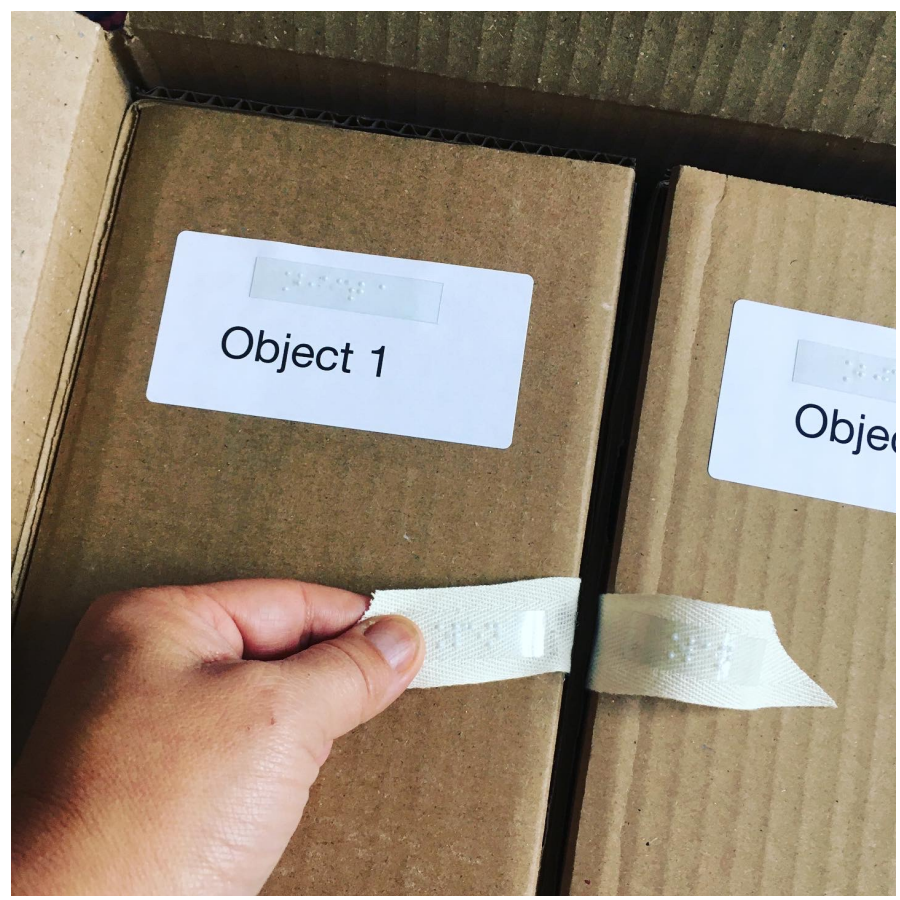

Note: Braille labels identified the contents of each box. On the fabric tabs to pull open each Object box, it is written 'open here'. 
Figure 10

'Museum-type' objects in kit

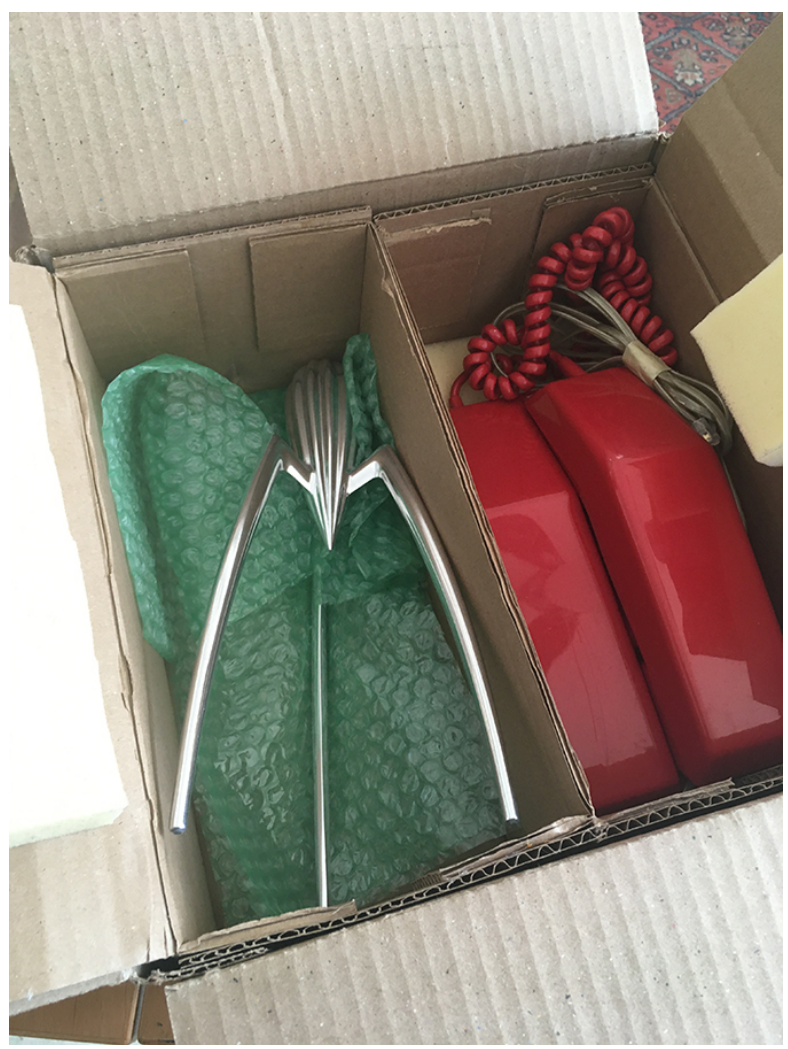

Note: Objects were protected for shipping used disposable bubble wrap that is easily identified as packing material.

\section{Figure 11}

Braille label identifies courier package as the research kit

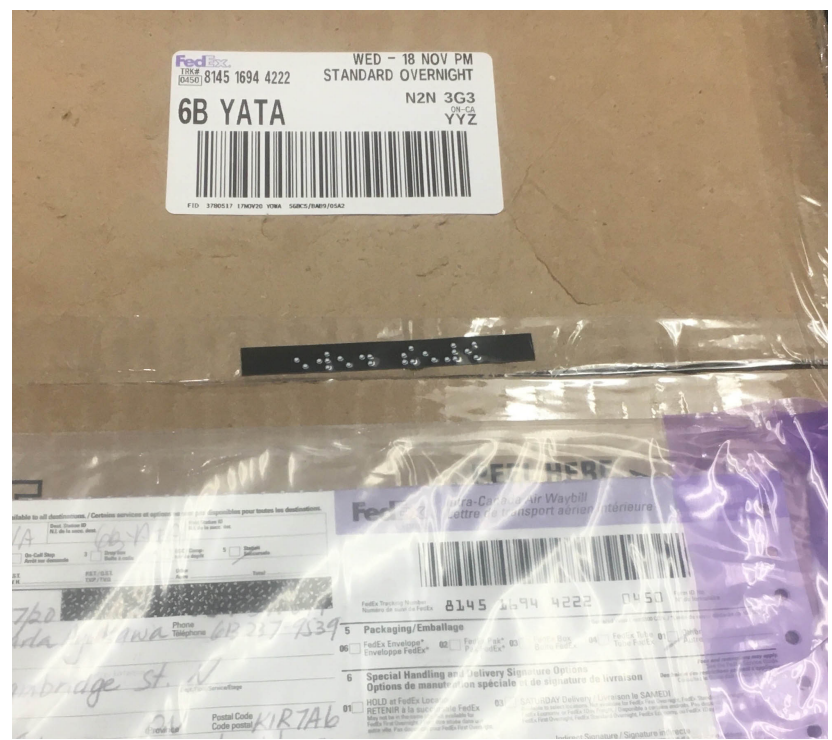

Note: A Braille label written 'research kit' was affixed on the exterior of the package. The label allowed the participant to identify the courier package. 


\subsubsection{The choice of objects}

The study used two commonplace objects with fundamentally different design approaches. Due to the unknown outcomes of the interdisciplinary methodology, two objects lowered the risk of not collecting useful data than if only one object was examined. The different design approaches inherent in the different objects also increased the likelihood of diverse responses from the participants. Lastly, objects associated with commonplace activities, like communication and food preparation, allowed for easy-to-understand functions and comments related to life experiences.

The objects for the study had to meet certain criteria. They needed to be: similar to objects found in museum collections; part of thematic exhibitions with ocularcentric narratives; robust (or replaceable) to allow for handling and shipping; able to be disinfected which was a concern for COVID-19 transmission at the time of the study; and available for the researcher to purchase. ${ }^{25}$

By requiring the object to have been exhibited as part of a prior thematic exhibition, the object was associated with an acknowledged narrative. Objects provide thematic exhibitions with context and evidence to help visitors understand the exhibition's narrative or storyline (Johnson, 2001). This is different than when objects are exhibited without an exhibition narrative, such as in an open storage exhibit display. In an open storage exhibit display visitors must form their own interpretation of the objects' societal relevance. For this study, the acknowledged and dominant object narratives acted as a baseline for comparing the new themes that could potentially emerge from the perspectives, insights, and stories I collected from participants.

Two objects met the criteria-Alessi's Juicy Salif (Figure 12) and Northern Research Electric Company's Contempra telephone (Figure 13). The Juicy Salif and the

\footnotetext{
25 The Juicy Salifs were purchased through Amazon.ca from Alessi and the Contempra telephones were purchased through second-hand retail.
} 
Contempra telephone are objects on the opposite ends of industrial design principlesthe telephone's design was motivated by function and efficient fabrication (Hadlaw, 2019), while the design of the lemon juicer was more focused on eliciting a visceral emotional response (Norman, 2004).

The Juicy Salif is part of museum collections of the Victoria and Albert Museum in London, England, the National Museum in Stockholm, and the Metropolitan Museum in New York (The MET). It is robust, easy to disinfect, and an example of an object associated with exhibitions linked to ocularcentric themes (Nationalmuseum, 2021; Vitra Schaudepot, 2020). The exhibitions After the Wall: Design since 1989 at the Vitra Design Museum in Weil am Rhein, Germany and 1989-Culture and Politics at the National Museum in Stockholm have all exhibited the juicer. In these contexts, it was used as an example of objects whose popularity was a result of its emotional design and the rise of a visual culture. ${ }^{26}$ These objects with photographic and catchy designs, gained a commercial advantage due to their unique physical appearance and the advent of the globalization of the press, Internet, and portable cellular technology. (Figures 14 to 17).

\footnotetext{
${ }^{26}$ Emotional design can be defined as a design phenomenon where the owners create a special attachment to an object after a process of emotional interactions that includes visual enticement, relationship, and fulfillment (Norman, 2004)
} 
Figure 12

Juicy Salif by Alessi

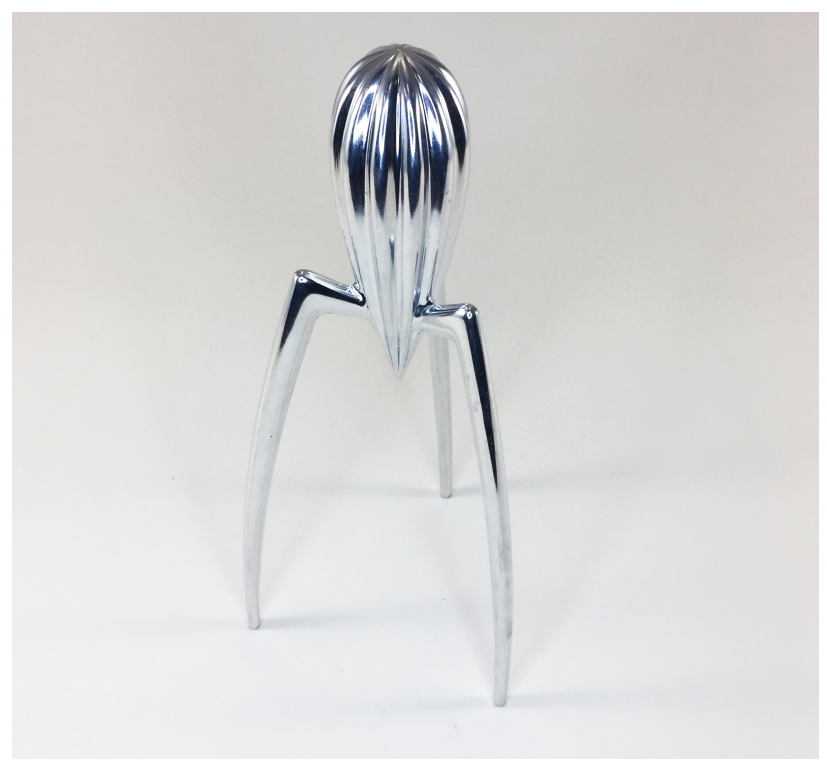

Note: The Juicy Salif is made of cast aluminum with a shiny bulb shaped ornament at its top and multiple ridges that taper down to form a point at the bottom. Three long thin legs extend off the bulb's point and then bends downward like an elbow where they form a tripod that supports the bulb. The thin metal legs resonate when tapped and the space between the legs fits a tall drinking glass. It is approximately $30 \mathrm{~cm}$ tall and $20 \mathrm{~cm}$ wide.

Figure 13

\section{Contempra telephone}

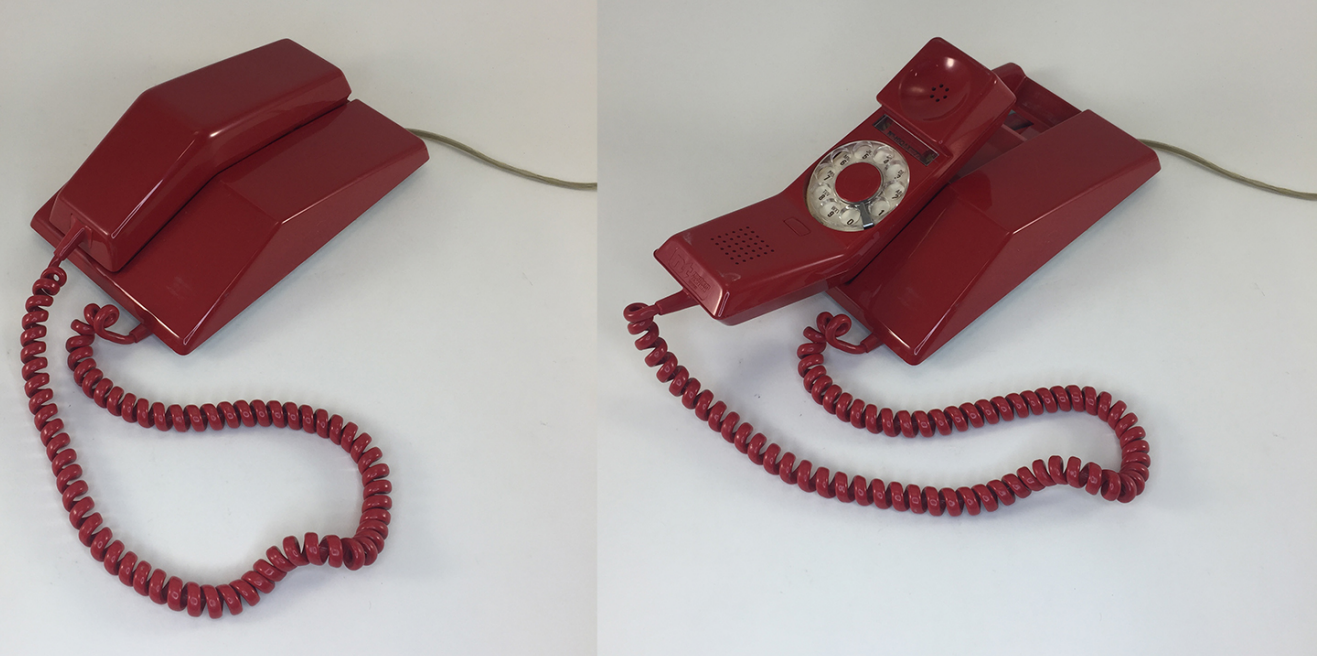

Note: The Contempra telephone is in closed and open positions. Its long, angular, and streamlined form, about the size of a paperback book is notably heavier when compared to today's telephones and its shiny yet featureless exterior makes it appear restful when placed on the tables or walls of homes and offices. 
Figure 14

After the Wall, installation view (Hunzinker, 2019)

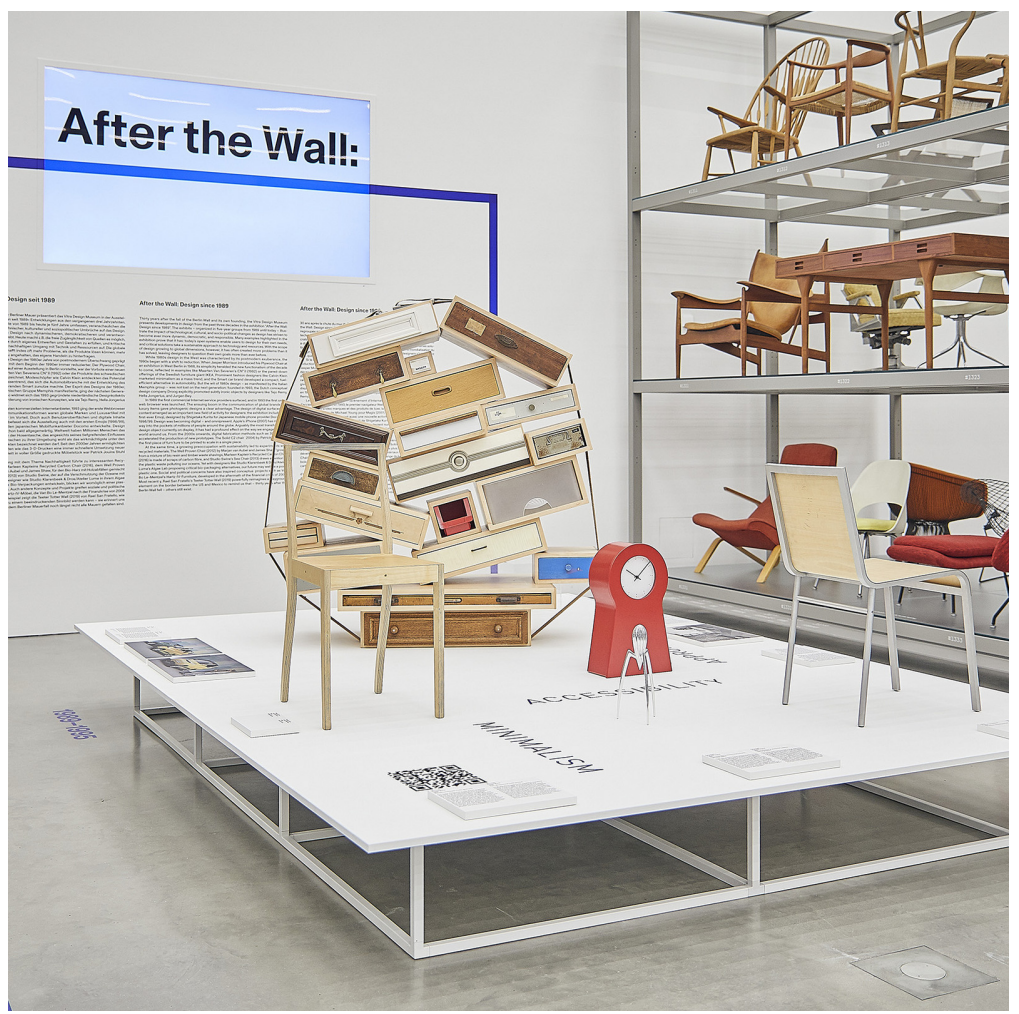

Note: The Juicy Salif was installed in the After the Wall exhibition at the Vitra Design Museum. It was presented on an open plinth adjacent to other objects that represented the visual culture of the 1989 era. On the horizontal surface of the plinth were the didactic panels. The object appeared to be approximately $1 \mathrm{~m}$ from the edge of the plinth, thus out of arm's reach from visitors.

Figure 15

After the Wall, exhibit detail (Stemmer, 2019)

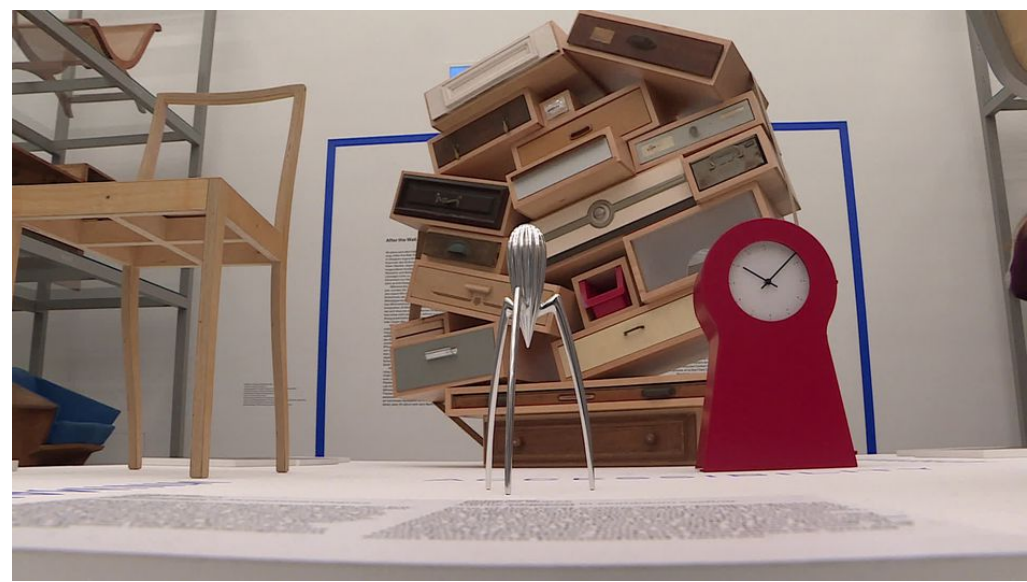

Note: The Juicy Salif was exhibited on a low plinth. The viewing angle of this image is almost parallel to the surface of the plinth and lower than accessible sightlines for visitors. 
Figure 16

1989-Culture and Politics, installation view (Löf, 2020)

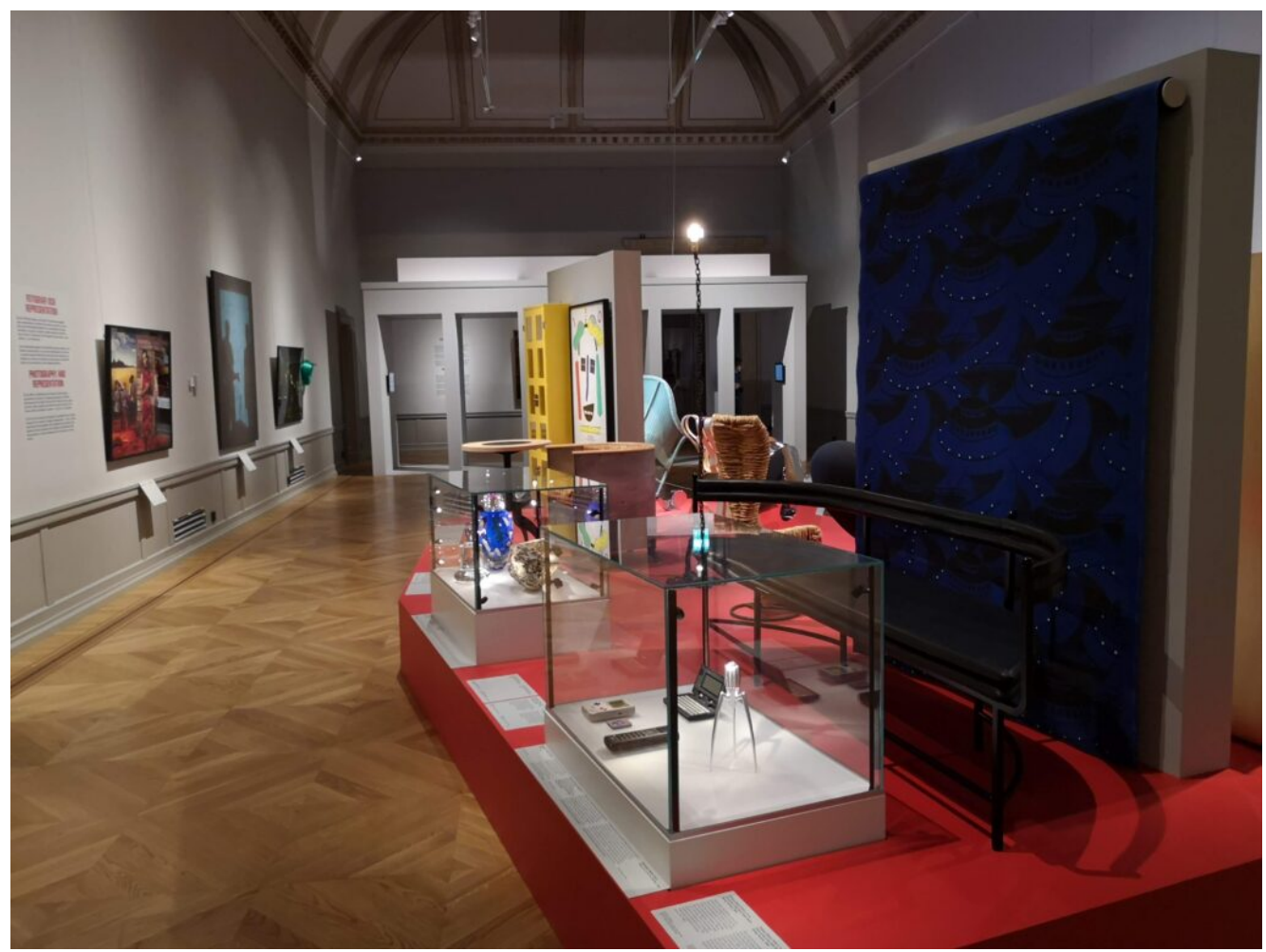

Note: This image shows the Juicy Salif exhibited at the 1989-Culture and Politics exhibition presented by the National Museum in Stockholm. The object was exhibited in a five-sided showcase. The height of the plinth was below waist height and the object labels were located in front.

\section{Figure 17}

1989-Culture and Politics, exhibit detail (National Museum Stockholm, 2019)

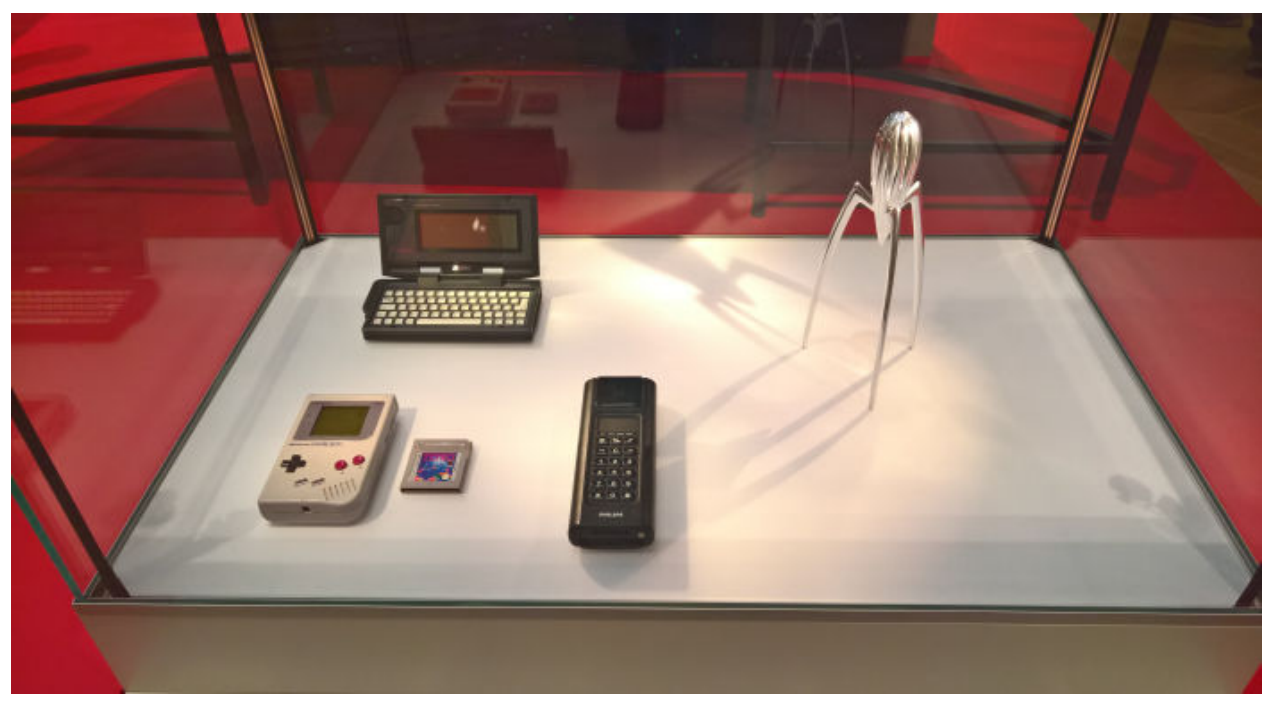

Note: The Juicy Salif was exhibited in the 1989-Culture and Politics exhibition. It was in a sealed showcase with four other objects. 
The Contempra telephone (Figure 13) can be found in the collections belonging to the Canadian Museum of History in Gatineau, Ingenium in Ottawa, and the National Museums in Scotland. The dominant curatorial narrative for thematic exhibitions that have exhibited the Contempra telephone is linked to the Canadian post-war modern design era and that era's use of plastic, colour (Hunchuck et al., 2006), retooled WWII factories, Scandinavian airiness (Collier, 2011), and the neo-platonic geometry of the International Style (Parr, 1999). ${ }^{27}$ Examples of the ocularcentric exhibition theme and exhibit design practices can be seen in the exhibitions The Modern Eye at the Art Gallery of Greater Victoria, Cool '6os at the Canadian Museum of History, and Classic Plastic at the Design Exchange museum. Figures 18-23 illustrate how the telephone was exhibited in all three exhibitions. The figures show each exhibition's traditional showcase/exhibit plinth technique in their exhibit design. Object labels were printed separately and, in some cases, at a distance from the object.

\footnotetext{
${ }^{27}$ Neo-platonic geometry is described as a design philosophy that values reduced clutter in details
} to reveal just the essential form (Parr, 1999). 
Figure 18

The Modern Eye, installation view (dominionmodern, 2013)

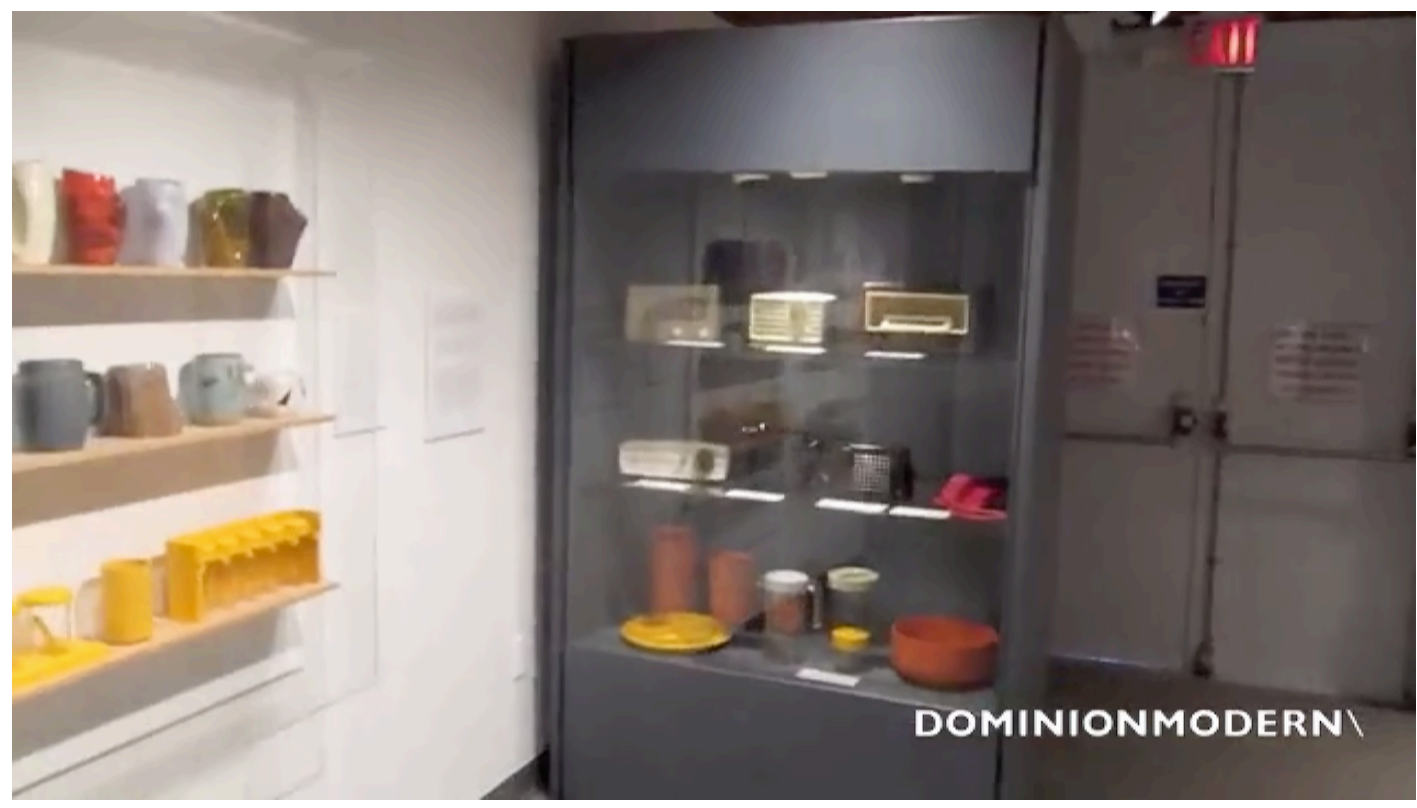

Note: The Contempra telephone was exhibited in a closed one-sided showcase.

Figure 19

The Modern Eye, exhibit detail (dominionmodern, 2013)

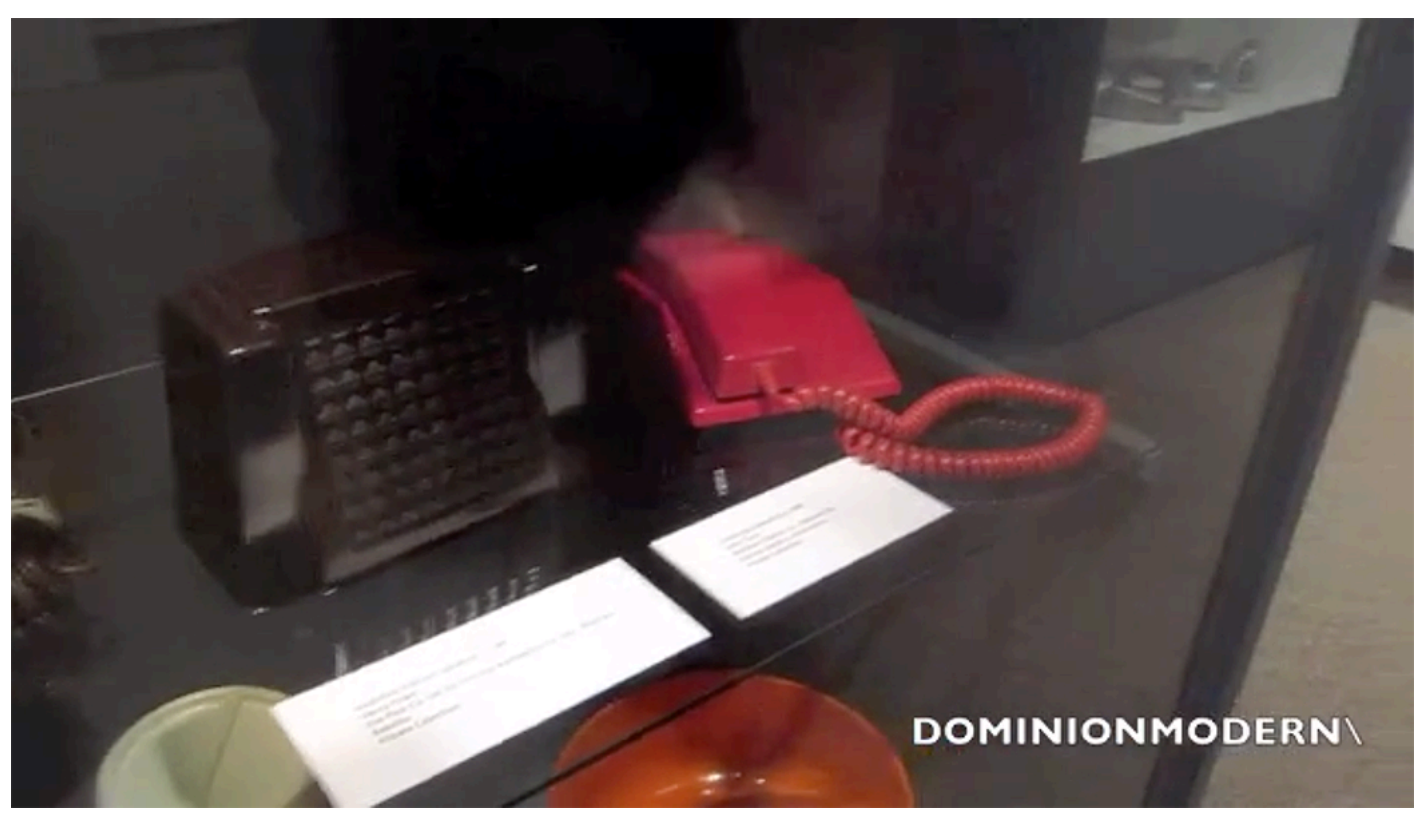

Note: A close-up of the showcase housing the Contempra telephone shows how the telephone was exhibited in the closed position and with an object label in front. 
Figure 20

Cool '60s, installation view (Hunchuck et al., 2006)

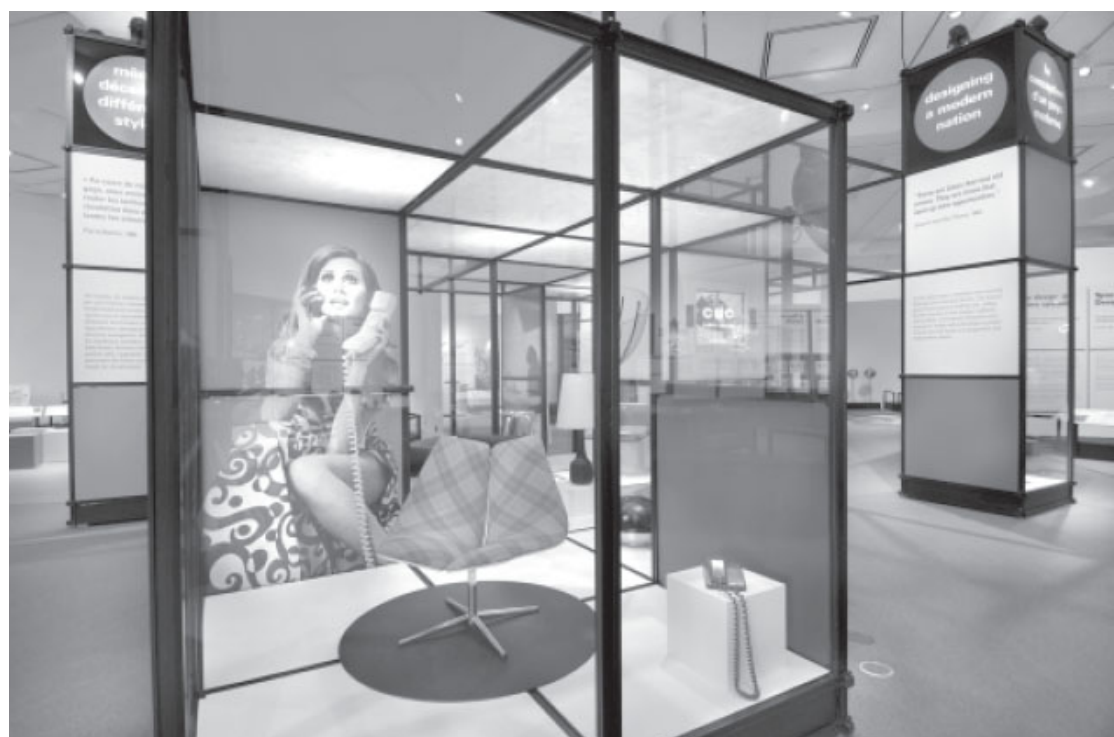

Note: The Contempra telephone was exhibited in a sealed Q-bit showcase system on a white plinth at waist height adjacent to another artifact, a bright orange lounge chair. The telephone was in a closed position and in the background was a showcase mural to provide context. The object labels are not visible from the angle of this photograph.

Figure 21

Cool '60s, showcase mural for Contempra telephone exhibit

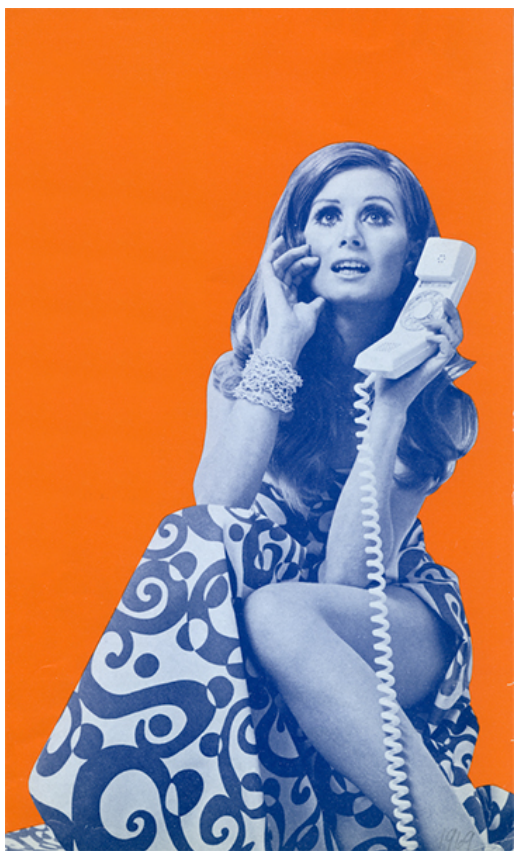

Note: This image of the showcase mural is a monochrome photograph of a female model holding the Contempra telephone in the open position. The background of the photograph is tinted bright orange that matches the colour of the lounge chair that was displayed adjacent to the telephone. 
Figure 22

DX Uncrated: Classic Plastics, installation view (Pesant, 2016)

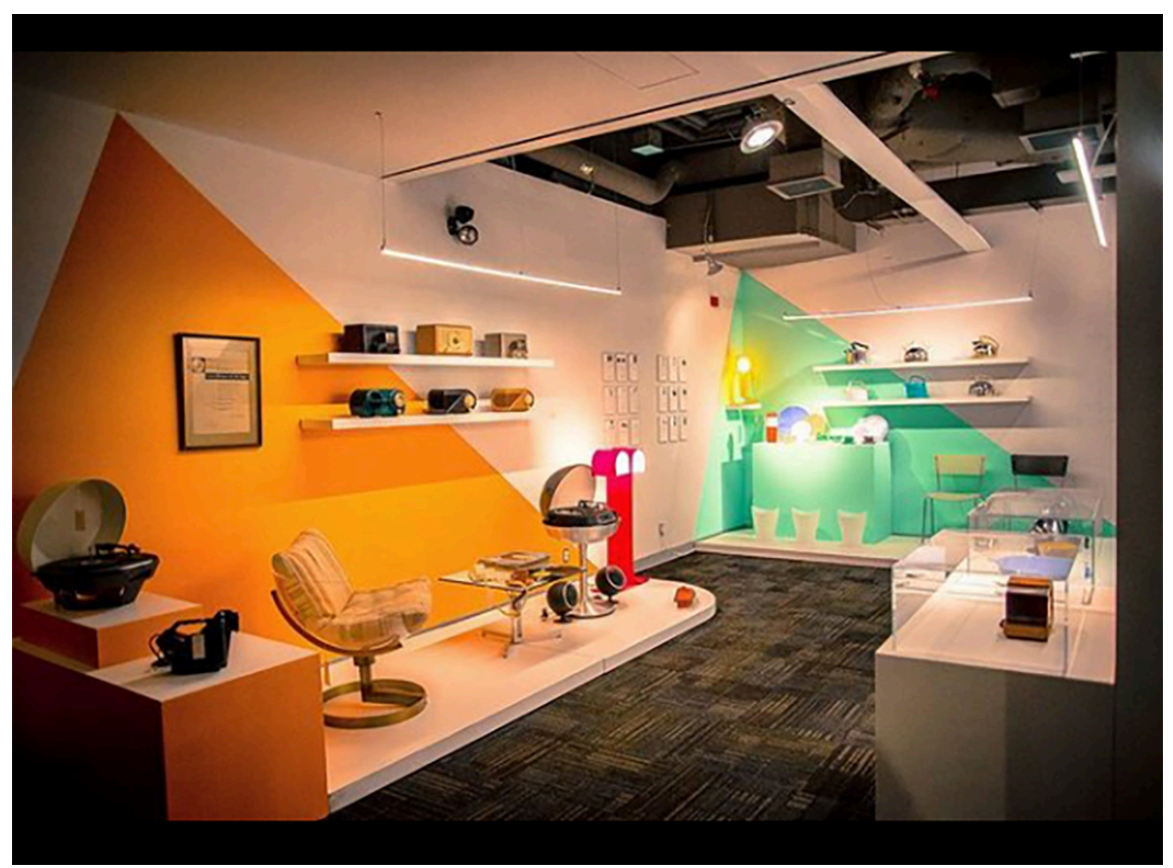

Note: This installation view of the DX Uncrated: Classic Plastics exhibition shows the Contempra telephone exhibited with objects that suggested a home environment from the 60s/70s era. It was displayed on a low table below waist height. Although the ensemble was installed on a plinth, the objects were within reach of visitors.

Figure 23

DX Uncrated: Classic Plastics, exhibit detail (Pesant, 2016)

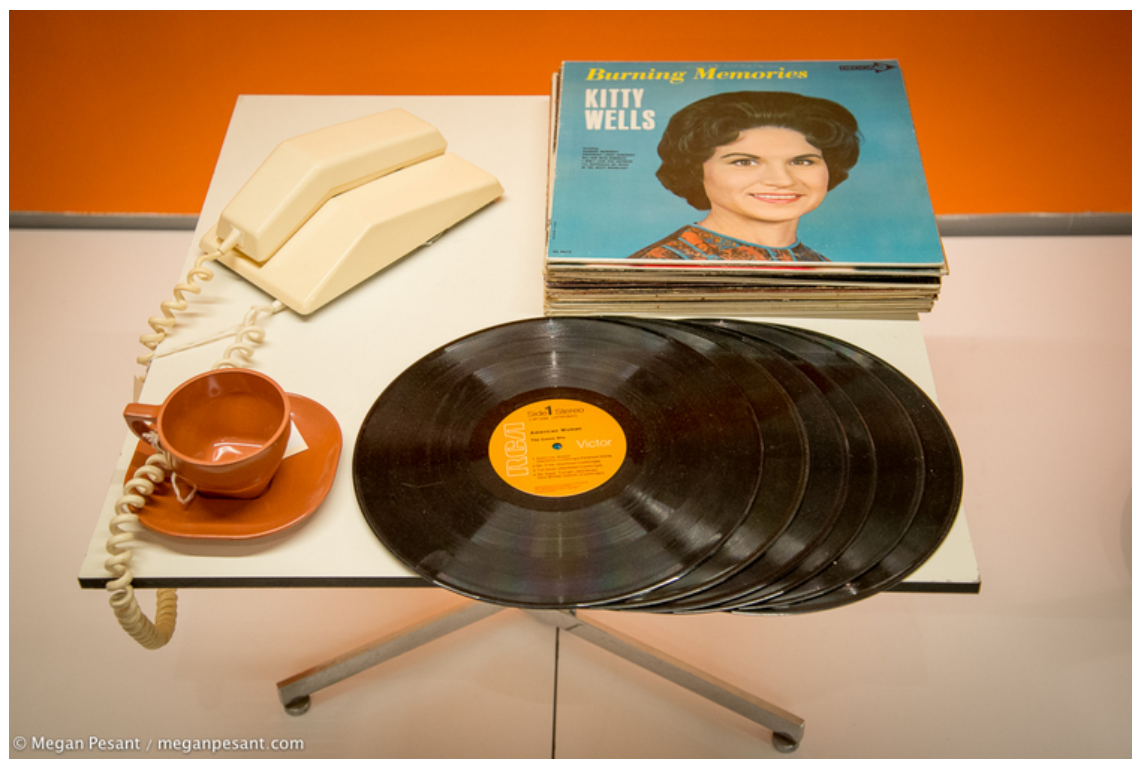

Note: The Contempra telephone was exhibited in a closed position with other objects from the same era. The colour of the telephone was almost identical to the surface colour of the table. 
These objects' narratives form a baseline to reflect on the findings that may emerge from participants' examinations of the objects, the thematic analysis, and the new proposed non-ocularcentric exhibit module. The new exhibit module that is developed from the study's findings is then compared in more detail to one of the dominant ocularcentric narratives of the Contempra phone described above (Cool ‘ $60 \mathrm{~s}$ ).

\subsection{Analysis and synthesis}

A variety of techniques were applied to manage, build familiarity with, and interpret the data collected from the object research sessions. This involved reflection and memoing, followed by building a thematic analysis using a more structured process

of in vivo coding. The inductive (bottom-up) and open-ended approach that thematic analysis offers provided flexibility in terms of allowing for the coding and interpretation of the verbatim transcription, which represented participants' individual experiences and outside the researcher's culture and personal familiarity (Robson \& McCartan, 2016). The process of in vivo coding offered a way into the participants' mindsets and experiences by preserving their actual words and voice (Saldaña, 2016), and perhaps serving to minimize misinterpretation caused by my bias.

\subsubsection{Reflection and memoing}

Immediately after each session, I reflected on the event with memos to identify emergent patterns, significant trends, ideas for themes, and insights (Robson \& McCartan, 2016; Saldaña, 2016). In memoing, I documented interesting activities from the sessions and noted questions and directions in the script that perhaps needed more clarification and improvement in subsequent sessions. The memos and a review of the session transcription informed any necessary operational changes for the next session. This process helped refine the ongoing approach to questioning (trying not to lead the participant) and direction (guiding the participants when discussions drifted outside the 
goals of the study). I also used the memoing exercise to help determine if I was reaching an acceptable level of saturation in the data (i.e., was I beginning to see similarities, patterns, and fewer outliers). The reflection and memoing also identified interesting, unexpected, and recurrent comments between different participants across sessions. Their comments inspired ideas for potential codes and early themes.

\subsubsection{Verbatim transcription}

In anticipation of in vivo coding, the audio recordings for each participant were transcribed verbatim. The participants' identifying data was removed, the timestamps preserved, and the transcripts were formatted into a Microsoft Excel spreadsheet. Separate columns in the spreadsheet were dedicated to different types of data. Each segment of the transcript was numbered sequentially to preserve the order of the data. The spreadsheet design allowed me to sort the data according to in vivo, initial, and focused codes, categories, and themes with ease. This was especially helpful when some in vivo codes crossed over to different categories. When the dialogue was ambiguous (for example, the participant describes something but it is unclear what they are touching), a description of the matching video supplemented the transcript. The process of verbatim transcription helped me build a familiarity with the data on a detailed and personal level which would later be fundamental to in vivo coding (Saldaña, 2016).

\subsubsection{In vivo coding and theming}

In this study the participants are considered a part of a cultural group who have distinct mental models of the world around them-perceptual expertise-and apply terminology differently than people with sight. The in vivo coding method helped to retain the participants' cultural perspectives and language which was crucial to defining an insight or impression (Saldaña, 2016) and to minimize misinterpretation caused by my ocularcentric bias. Using the sorting tool in the spreadsheet, similar codes were 
grouped together from which categories and themes emerged. The coding system and memoing assisted in identifying and confirming trends in common experiences and perspectives, sensory metaphors and descriptions, new insights, and other possible inclusive narratives.

Using the categories and themes that emerged from the coding, I started to formulate new possibilities for object narratives through an online whiteboard collaboration tool (Miro). Figures 24 and 25 illustrate how the themes for each exhibit were visually organized depending on if the object was either the subject of a theme or the object inspired a theme when it was used as a cultural probe. This exercise helped me interpret and visualize the theme and relationships among themes and subthemes or categories. Figures 28 and 29 show how images of the objects and supplementary objects (for example, props, graphics, or other artifacts) are added to each theme on the whiteboard to help define a theme. Six of the theme/exhibitions topics that emerged from the exercise are presented in Sections 4.3.1 and 4.3.2.

\subsubsection{Round-table discussion with participants}

An exhibit module (see Section 4.3.3) was conceptualized as a new cultural probe for a round-table discussion with the participant group. The purpose of the discussion was to consult on the inclusivity and accessibility of a theme/exhibition topic and design resulting from the study methodology. The theme/exhibition topic that was synthesized into an exhibit module (one of the Contempra phone themes/exhibition topics) was selected above the five other theme/exhibition topics because it bore the best attributes to answer the research question: 'How might we improve access to museum exhibitions through the exploration of inclusive narratives?'. These attributes included rich data that provided a variety of inclusive messages, robust opportunities for tactile and audio modes of content delivery, and ideas for accessible active learning components. It was 
also chosen because I had access to information related to the design of the exhibition, Cool '6os for comparison.

The materials that made up the exhibit module for MUSCLE MEMORY included the descriptions of the telephone's characteristics and the object's physical components that directly inspired the exhibition topic. For example, the movement of the rotary dial inspired the exhibition topic, therefore, the dial would be a component for delivering the exhibition message. All of the components that made up the exhibit module's design were non-visual. For example, rather than a visual didactic panel to deliver the content, audio delivery was prioritized. The audio mode of delivery was a core part of the exhibit design meaning: it was not an accessibility accommodation added on to support or animate a graphic panel after the fact.

In a Zoom meeting, exhibit module was described to participant group who were then invited to comment on the design and exhibit topic. The first set of questions focused on the exhibit module. The second set of questions focused on the study's consultation process (the focus groups and the round-table discussion). For both sets of questions, the participants were asked what they liked the most, what they liked the least, and to offer suggestions to make it a better experience.

The following is a summary of the script for the round-table discussion where the exhibit module was presented to the participant group. The goal of the discussion was to hear their comments on the proposed inclusivity and accessibility of the exhibit module and if it might create an environment where visitors could have a shared museum experience. A full version of the script is available in Appendix C.

\section{Round table discussion-summary of the script}

1) Purpose of discussion.

2) Background of events leading up to round table discussion.

3) The exhibit's Big Idea and its extended messages.

4) How does the Contempra telephone fits in? 
5) Encountering the exhibit as a visitor.

6) The entire exhibit would be designed to be audio and tactile-based.

Figure 24

Schematic of visual organization of theme relationships
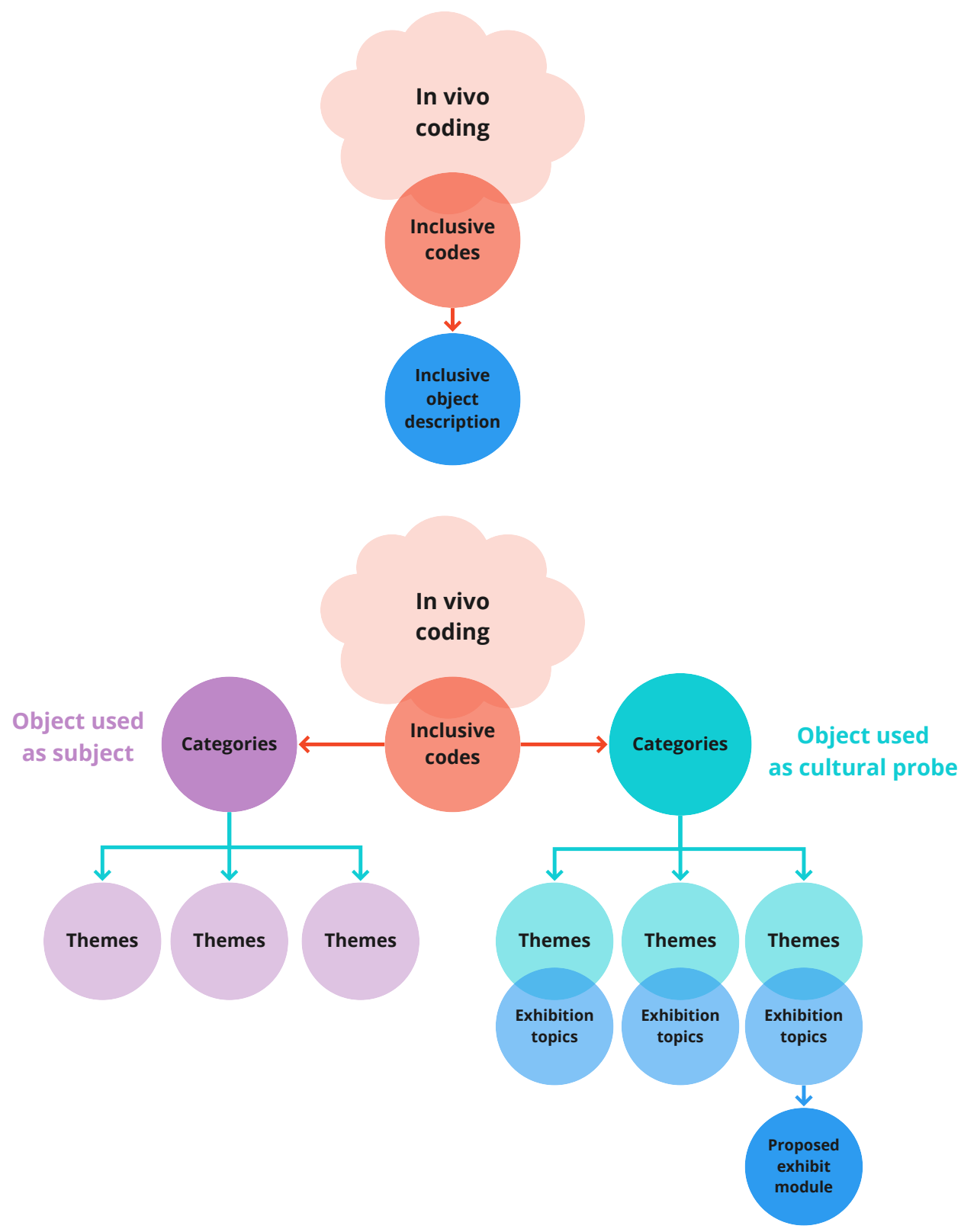

Note: This schematic shows how the different themes for exhibits were visually organized. The in vivo coding was identified as a description or a narrative. Within the coding for narratives, the categories were characterized whether they emerged from the telephone used as the subject or as a cultural probe. From the categories, themes emerged. One of the themes was synthesized into an exhibit module. 
The methodology offered an interdisciplinary practice and remote research opportunity with participants who have distinct knowledge. Using the object research sessions and verbatim transcripts, I collected data that could perhaps contribute to minimizing sensory and experiential barriers in exhibit design. The necessity for remote research added a layer of complexity in data collection by requiring the development of an accessible research kit within the unique context of one's home. However, the development of the remote research kit contributed positively to recruiting a larger sample of participants (due to the local rarity of congenital and early-onset blindness) as well as more tailored focus group pairings. 
Visualization of analysis on MIRO

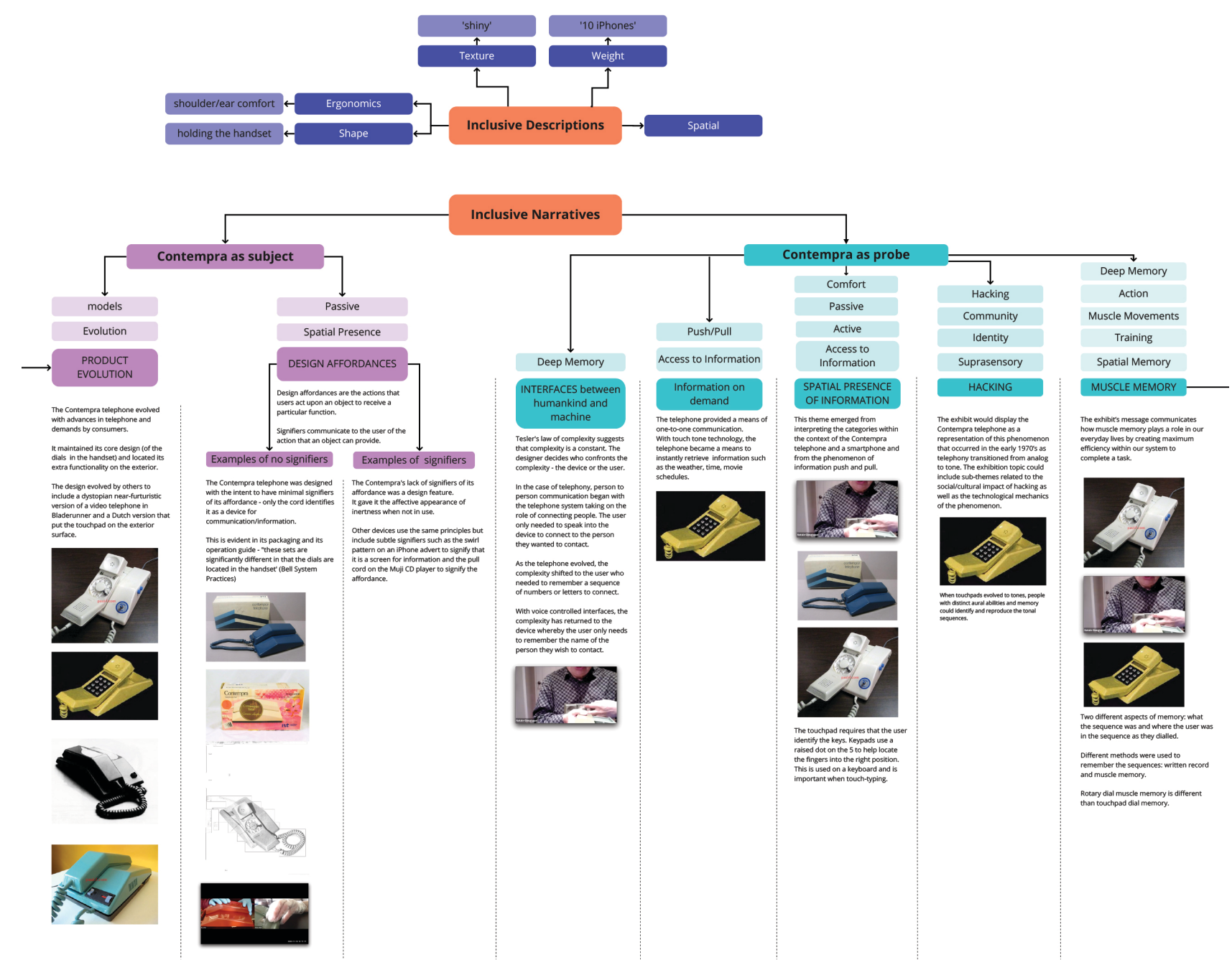

Note: This image is a screen-capture of the Miro whiteboard used to visualize, understand, and realize new possibilities for narratives. 


\section{Findings}

In this chapter, I share the findings from the study and use these findings to explore a strategy to develop inclusive narratives and accessible exhibit designs. These findings include the results that emerged from analyzing the data from the object research sessions, the insights related to this analysis, and the application of results toward an inclusive and accessible exhibit module. The findings will help to inform future studies when collaborating with participant groups (including and not limited to people with vision loss) to conduct object research and develop accessible exhibitions.

The findings provided in this chapter have been organized into three sections. The first section provides insights about the operations of the object research session that concern the participant research experience, interrelationships among participants, their use of nitrile gloves, and their use of ocularcentric terms during the sessions. These findings are important to report since they provide insight into the workings of object research sessions with the blind community. The second section provides an account of the experiences unique to participants who are blind when conducting remote research. These findings are valuable to share since remote research may evolve post-pandemic to support greater participation of communities who are not located in one region, and in turn, lead to more inclusive study designs or exhibit development practice. In the third section, I describe the themes/exhibition topics that emerged from the thematic analysis, how they informed the development of an exhibit module, and the participants' thoughts on the exhibit module.

\subsection{The object research session-how it went}

The experience involving the main actors and components of the object research session-the participant, the 'museum-type' objects, and the script-led to insights that may be relevant in developing future object research sessions. These experiences 
involved participants' biases and interrelationships due to previous participation in research studies, participants' reactions to wearing nitrile gloves, and the participants' use of ocularcentric expressions.

\subsubsection{Participant bias}

Some participants commented that they often participate in research studies out of personal interest or to supplement their income. Participants $\mathrm{OH}$ and $\mathrm{EH}$, for example, were experienced in the field of product testing and that seemed to influence their responses. Their discussions about the Juicy Salif focused on redesigning the object to suit their personal needs rather than providing their impressions about the object. Because of their preoccupation with testing an object's function, it was difficult to redirect their conversation to discuss its design and social significance. The data collected from these participants was sometimes lacking commentary beyond functional attributes, however, these two participants did offer in-depth descriptions in other areas such as how to operate a rotary dial telephone. This data was coded and interpreted in categories such as action and muscle movements.

\subsubsection{Insights about the focus group pairings}

There was evidence to suggest that attention should be given to creating compatible pairings in focus groups because of the small size of the source community. In almost all the focus group pairings, the participants were acquainted socially, professionally, or through common circumstances such as attending the same school. In one pre-screening interview, a participant spoke out that they preferred not to be paired with a particular individual due to poor experiences in the past. As a result, this participant was paired with someone (a stranger but with mutual friends) from a different city and who shared a spiritual affinity (they both were active members of their churches). Later, the participant declined to participate in the round-table discussion to 
avoid contact with the same person. Future research studies may need to take into consideration participants' pre-existing interrelationships when planning their study with tight-knit communities.

\subsubsection{Insights about the object examination and tactility}

The participants were very engaged when examining the objects and generally receptive to directions except when it came to wearing nitrile gloves. The requirement for wearing nitrile gloves was met with some resistance, and in one case, defiance due to their loss of tactility and decreased ability to 'see' the object. This loss was evident when the participants removed the gloves and discovered the neoprene feet on the Juicy Salif's legs. The discovery of the neoprene made it clear to the participants which way was 'up' for the Juicy Salif. By not knowing which way was up some participants held the juicer by the bulb when commenting on and interpreting the object as illustrated in the righthand photo in Figure 26. When another focus group discussed how the shape of the bulb was like an ice cream cone, there was confusion and differences in impressions due to the different orientations of the object.

Figure 26

Holding Juicy Salif two different ways

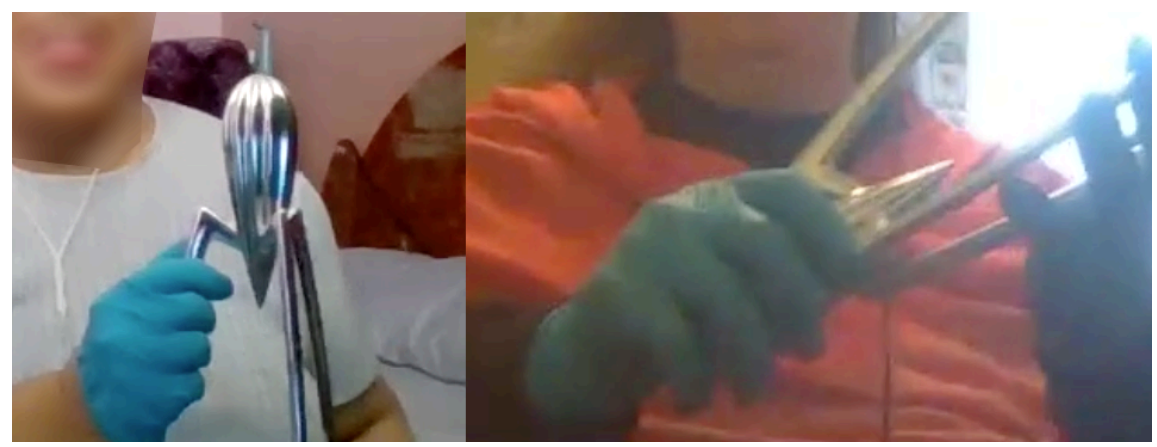

Note: This screen-capture shows two participants holding the juicer in two different orientations. One participant is holding it by one of its legs and the other by the bulb. Some instinctively held the juicer by the bulb because of its shape and weight. Others were drawn to the tripod legs and stood it on the table. Participants' interpretations and impressions of the juicer differed depending on how they were holding the object. When participants felt the neoprene pads at the end of each 'leg', they were able to confirm the orientation. 
The object examination also demonstrated the participants' tactile abilities. When not wearing the gloves, they detected and some read the letters cast into one of the legs of the Juicy Salif. Figure 27 illustrates the level of detail in the letters cast into the juicer's leg.

Figure 27

Sensory tactile detection

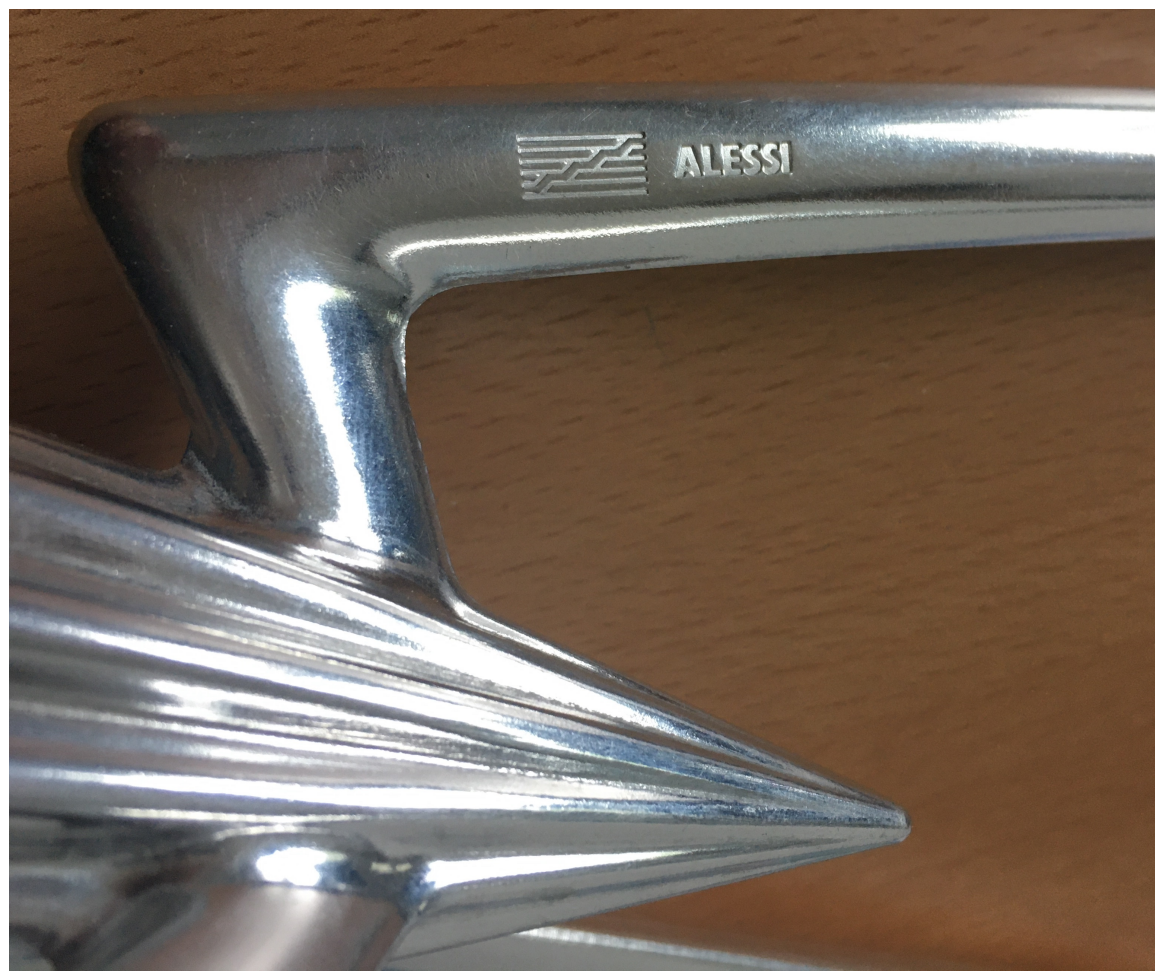

Note: This close-up image shows the ALESSI logo cast into the juicer. Some of the participants, when not wearing gloves were able to tactilely read the letters. This is an example of how sensory abilities were dulled when wearing nitrile gloves.

\subsubsection{Insights about the object research script}

The script evolved after each session as a result of the reflection and memoing exercise. During this exercise, I noted instances of unclear questions, reoccurring responses, and participants' use of ocularcentric words. For example, it was common to hear ocularcentric phrases such as "It looks like it is shiny" (Participant LC), "You can see the tiny details without the gloves" (Participant $\mathrm{OH}$ ) or "It has a restful 
appearance" (Participant DN). I had an initial reaction of confusion when I heard comments such "I have never seen one with a circular face" (Participant DN) or an exchange between two participants: "[The telephone] could mount either way. [Do] you see the hole on the bottom?" (Participant $\mathrm{NH}$ ) “Oh, that's right. I see it. Yeah." (Participant EH). Later, I interpreted words such as 'seeing' and 'looking' as expressions that could suggest understanding through 'feeling' and 'touching' and adjectives like 'shiny' were understood to imply surface texture and not light reflection. This early confusion could have been the result of my ocularcentric bias and an assumption that their language defaults to non-visual verbs. Adjusting my mindset about 'ocularcentric' words and being more attentive to the potential for their figurative meaning in the discussions, made responding to and directing participants easier. For example, when inquiring why a participant described an object as 'classy', I better understood their response: "It has a shiny feel ... it looks crafted", (Participant LC).

\subsection{Insights about conducting remote research}

Remote research with the participant group revealed some unexpected experiences related to couriering and the time of day in which the recording took place. The incidents did not affect the data collection, however, knowledge about these experiences may contribute to improving future remote research planning and process.

\subsubsection{Insights about the couriers}

The contactless delivery by couriers resulting from COVID-19 prevention protocols meant that sometimes participants were not notified of a delivery, or the package was placed in unexpected locations. Consequently, some participants expressed frustration in locating the package. On one occasion the package was found a day after it was delivered underneath the snow, which permanently damaged the packaging. In another incident, the participant mistook a random box they found on their porch for the 
courier package. Although the delivery instructions were included on the courier delivery slip, these experiences reveal the challenges people who are blind frequently encounter and the importance of designing a very detailed process, with redundancies to support future remote research with this community.

\subsubsection{Insights about the time-of-day recording}

The time-of-day recording revealed another unexpected consideration when conducting remote research with participants who are blind. The research sessions were conducted in the morning except for the last focus group. Session 6 was in February at 4 $\mathrm{pm}$ and the quality of the video recording was affected by winter's early sunset. At the beginning of the session, the participant was illuminated by the sun and by the end of the session, they were in complete darkness. If this had occurred in an earlier session, a direction would have been added to the script to ask the participant to 'turn on the lights if possible' or to 'use the battery-operated light included in the research kit'. Such factors again point to the importance of understanding subtle nuances and contextual considerations in designing remote object research sessions.

\subsubsection{Premature access to research kit}

As part of the findings and important to future research designs, some participants opened the research kit before the object research session interview. While it was not stipulated in any instructions to not open the research kit, some participants may have had prior knowledge of the contents which may have influenced their comments.

\subsection{Inclusive narratives and accessible exhibitions}

This section provides the results that emerged from the reflection and memoing exercise, verbatim transcription, coding, and thematic analysis. Through the examination and interpretation of the data, several useful initial codes emerged and, in 
some cases, acted as a starting point for inclusive exhibition topics. Those codes led to categories that were used to reexamine the data again to generate and assess the strength of the theme. The results from this work led to the proposed inclusive exhibition topics and accessible exhibit design concepts which were then shared with the participant group for feedback.

The object descriptions from the data and in vivo excerpts helped me generate ideas for multimodal exhibit interactive tools with the intent of supporting meaningful exhibition content delivery for visitors with vision loss. The interactive tools were intended to be inherently accessible for delivering the exhibit message.

I identified various initial codes as significant and 'bucketed' these codes into categories. The types of categories were based on how they represent the physical or aesthetic characteristics of the object, impressions about the object, lifestyle associated with the object, personal stories about the object or related to it, and comments about its use or function. More focused codes and categories were generated and interpreted into higher-order themes intended to be inclusive of a general visitor audience. Each exhibition topic was organized, defined, and illustrated in a Miro whiteboard describing how the museum object plays a role in the exhibit message. Figures 28 and 29 illustrate how the themes that emerged from the data were visually organized and defined into exhibition topics. 


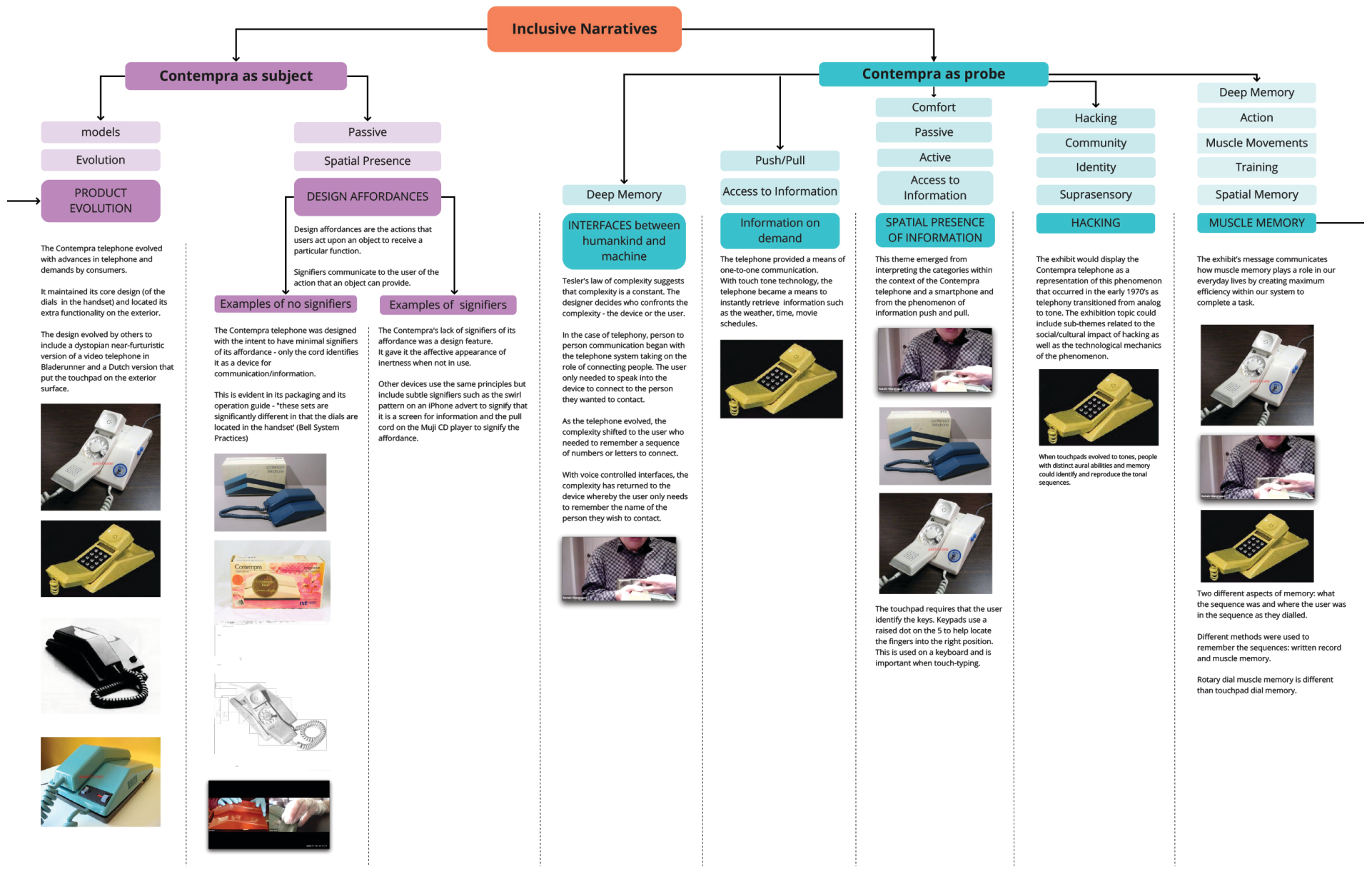

Note: This figure shows inclusive narratives organized into two groups. The group on the left in purple considered exhibition topics where the telephone was the focal point. The second group on the right in blue illustrates topics that emerged when the telephone was used as a cultural probe. The following figure, Figure 28 , offers a detailed view of the second group of topics. 
Figure 29

Visual organization of themes and inclusive exhibition topics

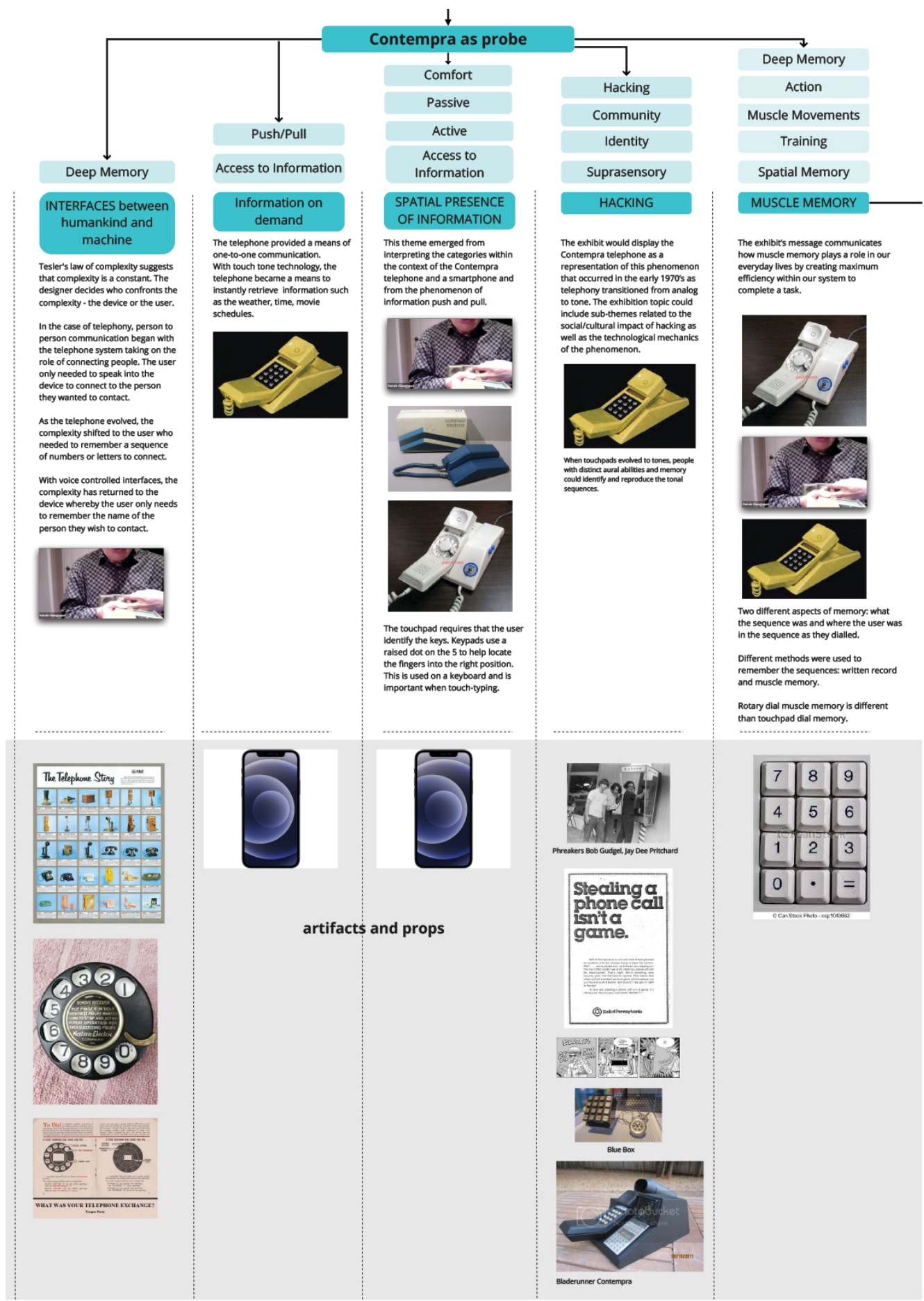

Note: This figure shows the categories and themes organized in columns with brief descriptions of the exhibition message and below images of artifacts or props to provide context to the messages. For example, in the column about the theme of HACKING, images representing subthemes about phone phreaking [hacking], identity, community, repurposing, and suprasensory are included. 


\subsubsection{Themes and exhibition topics related to the Juicy Salif}

This section provides an overview and examples of the diverse themes/exhibition topics drawn from the analysis of the comments about the Juicy Salif. The comments were coded and categorized to reveal themes that were then interpreted into inclusive exhibition topics that may offer a common point of access to a general visitor audience.

\section{Theme/exhibition topic A-THE VICTORIAN ERA AESTHETIC}

This theme speaks about the Victorian era aesthetic which emerged from initial in vivo codes such as bric-a-brac and altar boy stuff and sorted into categories such as old and antique but also impressions and experiences related to craftsmanship, tradition, and social class, which were drawn from comments about the object's physical and aesthetic characteristics (Box 1). From the analysis, the theme or exhibition topic about the Victorian era aesthetic emerged. It was defined as the era before the widespread adoption of mass-production and assembly-line factory practices. Some of the codes literally contained the words Victorian era. Discussions around the juicer also led to codes that included language such as door-knockers, clutter, and housework. Some of the impressions and perspectives contained language such as ornamental and beveling, and included references to their mother and Catholic liturgical items.

From this analysis, the question that arises in this exhibition topic is 'What makes something a VICTORIAN ERA AESTHETIC? Victorian originally refers to a particular time period -1837 to 1901 . However, the term 'Victorian' design frequently harkens back to a particular emotional feeling or lifestyle and it has been modernized into a neofuturistic or steampunk aesthetic. ${ }^{28}$ The Juicy Salif is an object that can represent 'Victorian' even though its outward appearance does not include any of the visual Victorian era stereotypes.

\footnotetext{
${ }^{28}$ Steampunk is defined as an aesthetic that includes neo-Victorianism, techno-fantasy, and retrofuturism (Perschon, 2012).
} 
Box 1

Example of thematic coding analysis leading to topic $A$

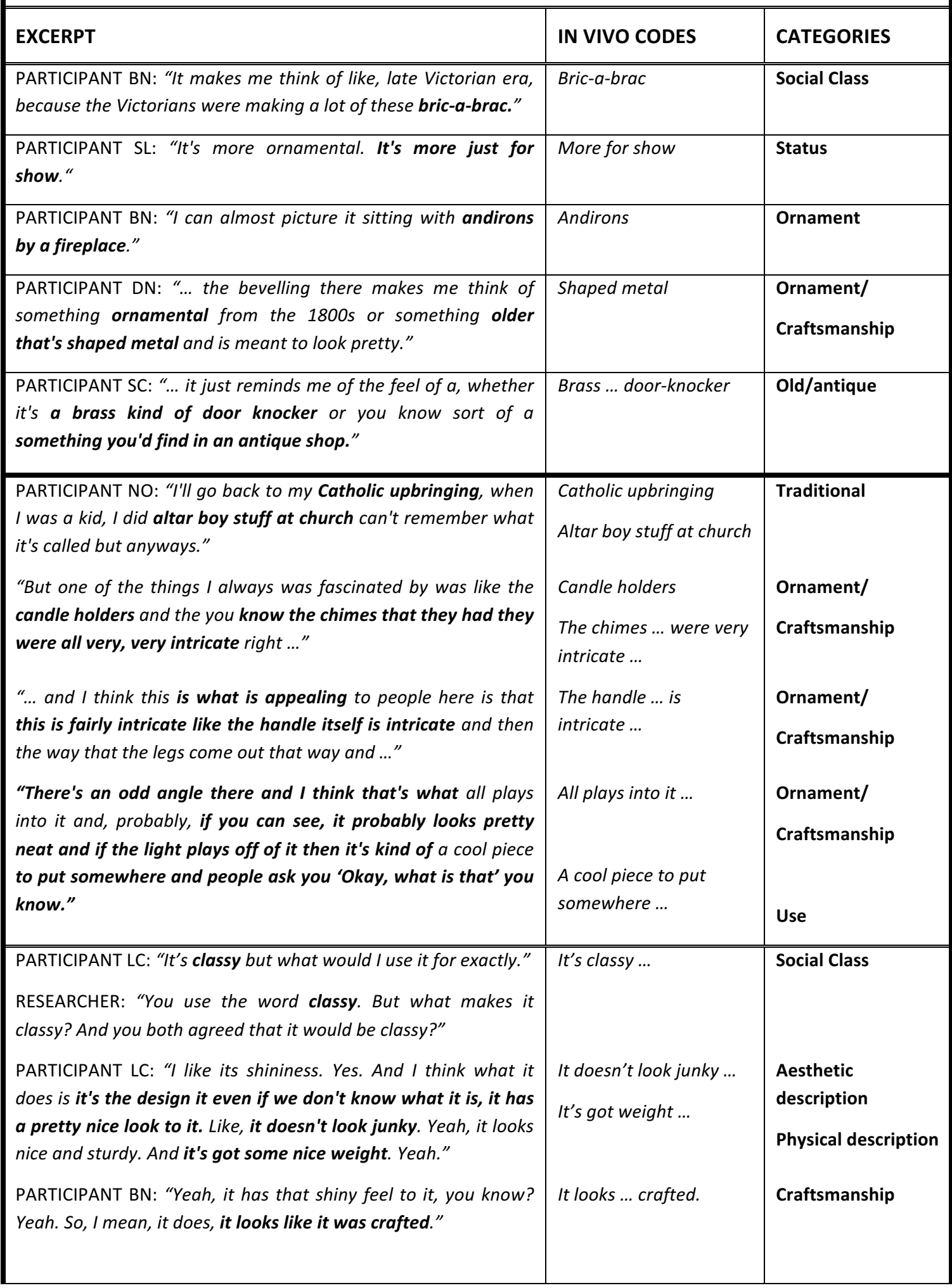




\begin{tabular}{|c|c|c|}
\hline EXCERPT & IN VIVO CODES & CATEGORIES \\
\hline $\begin{array}{l}\text { "They used to make things better in especially when ... I mean, } \\
\text { even before the Industrial Revolution in the early } 1800 \text { s got } \\
\text { started. But even as it progressed, they were still crafting } \\
\text { things. You know, I mean, if you look at designs and pictures of } \\
\text { different things, even doorways on, say the Titanic even. Yeah, } \\
\text { those people were proud of the work they did." } \\
\text { "You know, and so it's a tragedy it went down in that way. Like } \\
\text { all other historical discussion, I'm a Titanic buff, but I mean, it's } \\
\text { just you think of all the moldings and the crafting, I could see it } \\
\text { on the Titanic, you know, just something nice and classy that } \\
\text { they would use and it's even the most basic implement there } \\
\text { was it was crafted." } \\
\text { "And now you buy stuff, you know, it's a piece of crap that } \\
\text { machine cranked out, you know, so, but they, they were they } \\
\text { crafted things back then. Even going into the early } 20 \text { th } \\
\text { century. They were still." } \\
\text { RESEARCHER: "So this reminds you of something that was } \\
\text { crafted versus manufactured?" } \\
\text { PARTICIPANT LC: "Correct, potentially." } \\
\text { PARTICIPANT BN: "Well, even eventually, potentially, even if it } \\
\text { was manufactured, it was done so by craftsmen." }\end{array}$ & $\begin{array}{l}\text { On the Titanic ... } \\
\text { Something nice and } \\
\text { classy ... } \\
\text { They crafted things } \\
\text { back then. }\end{array}$ & $\begin{array}{l}\text { Craftsmanship } \\
\text { Old/antique }\end{array}$ \\
\hline
\end{tabular}




\section{Theme/exhibition topic B-SPATIAL PRESENCE}

The theme for this exhibition topic emerged from codes that identified language that pointed to the importance of the volume of space an object occupies as well as its relationship with the space around it. For example, the participants were conscious and curious about the negative space between the prongs or legs and commented about putting something in the middle. It was characterized as having a spatial presence. The language in the in vivo codes such as lives, approach it, and prominence supported that category. The participants were also conscious of its ornamental aesthetic as a centerpiece and its impracticality of size compared to its humble function. The juicer was described as an object that is living and almost has a personality or prominence beyond being a tool as it consumes a space or surface (Box 2).

The exhibition topic of SPATIAL PRESENCE can be defined as an aesthetic characteristic of an object where its identity goes beyond its physical shape to include the space around it and the position it holds relative to other objects in its presence. The Juicy Salif represents this phenomenon where the space between its three legs and the space that its aura occupies are as much a part of its identity when it is in use as when it is not. 
Box 2

Example of thematic coding analysis leading to topic $B$

EXCERPT

PARTICIPANT DN: "So my lemon squeezer is very efficient. And it's about, you know, eight inches long, and it's totally efficient. And it's tucked in my drawer."

"This you don't put this in a drawer, you put it on your marble, or your granite countertop and you approach it."

"I think that's part of it is like it's the kitchen where all of your kitchen where it looks beautiful, and you approach the lemon squeezer."

PARTICIPANT TX: "This is actually a designer lemon squeezer."

PARTICIPANT DN: "That's amazing. So yeah, it's the substantialness of it, the weight, the heft, and the fact that it's a self-supporting object that you approach."

"I'm sure that's part of the cachet of it is that it's, you know, you could picture this on your granite countertop in your island and your enormous kitchen because you have so much space."

"Not mine, not on my kitchen. But you have so the I feel like the implication is, you have so much space in your, you have so much space in your palatial kitchen that you can afford to devote counter space to your lemon squeezer."

"It's an object that just lives on your countertop."

"So, I feel like it's a expression of affluence, because you don't have to tuck your lemon squeezer away in your drawer you can afford you have so much space, it can be its own thing on your granite countertop."

"That's why I think and it's so heavy, that it's not, it's not going to blow away when you're, you know, your microwave blows on it or something."

RESEARCHER: "When you said it lives on your, on your countertop, what do you mean by like, can you talk more about that, I like the word that you said the word lives. Like how?"

PARTICIPANT DN: "It's more than a tool."

"But this is obviously this is obviously bigger and more ornate than you need for the purpose. And so, by making it it's a statement to make it so big, and so consuming of space. It's a statement that I have so much space."

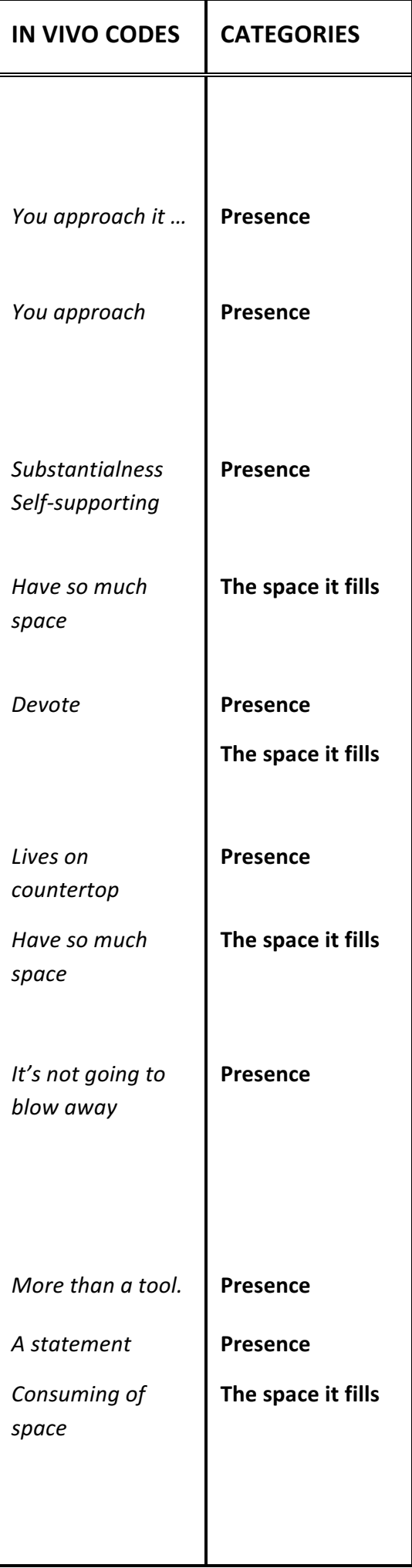




\begin{tabular}{|c|c|c|}
\hline EXCERPT & IN VIVO CODES & CATEGORIES \\
\hline $\begin{array}{l}\text { "So, it's an It's so heavy, and it doesn't need to be made of metal it could } \\
\text { be made of you know, you can have a plastic lemon squeezer, it's just way } \\
\text { more than it's overkill for the function. And so, when you make } \\
\text { something overkill for its function, I feel like you're trying to give it life or } \\
\text { value that it doesn't inherently have." } \\
\text { "So, when I say lives, it's like, you can't just toss this aside, it's so } \\
\text { important that it has a life of its own." } \\
\text { "And probably I guess that's how upscale kitchen places market } \\
\text { themselves as 'our stuff is just so great. It has a life of its own." }\end{array}$ & $\begin{array}{l}\text { Overkill for } \\
\text { function } \\
\text { Give it life } \\
\text { Has a life } \\
\text { Has a life }\end{array}$ & $\begin{array}{l}\text { Presence } \\
\text { Presence }\end{array}$ \\
\hline $\begin{array}{l}\text { PARTICIPANT SL: "And I initially thought that this was some sort of a } \\
\text { tripod. So that it like it stands on three legs. But then when you feel the } \\
\text { top then it's like no, no, I don't think something could be on the top of } \\
\text { this." } \\
\text { "It seems like that might be the centerpiece. So, this stands up. And then } \\
\text { it's got the legs just to give it some prominence and whatever this little } \\
\text { ornamental thing on the top is. That's, you know, what this was supposed } \\
\text { to do is just showcase it." }\end{array}$ & $\begin{array}{l}\text { Centerpiece } \\
\text { Prominence } \\
\text { Showcase }\end{array}$ & Presence \\
\hline $\begin{array}{l}\text { "But I like the idea how }[\mathrm{OH}] \text { was saying that. Like, if you put this on a } \\
\text { table, you could put something in the middle of these three legs." } \\
\text { "So, something, something could go under it. So maybe like a little } \\
\text { flowerpot or some sort of an ornament to kind of accent it." }\end{array}$ & $\begin{array}{l}\text { Something in the } \\
\text { middle } \\
\text { Under it }\end{array}$ & $\begin{array}{l}\text { The negative } \\
\text { space } \\
\text { The negative } \\
\text { space }\end{array}$ \\
\hline $\begin{array}{l}\text { PARTICIPANT EH: "And I don't like that you can't store it isn't the best, but } \\
\text { they don't want you to store it." }\end{array}$ & $\begin{array}{l}\text { Don't want you to } \\
\text { store it. }\end{array}$ & The space it fills \\
\hline "They wanted to keep it on your counter and use it forever, right?" & Keep on counter & Presence \\
\hline $\begin{array}{l}\text { PARTICIPANT EH: "It's not really designed to be handed from hand to } \\
\text { hand. So, you want to keep it stationary." }\end{array}$ & Keep it stationary & Presence \\
\hline $\begin{array}{l}\text { PARTICIPANT NO: "So for me, the first thing that I thought of was I was } \\
\text { barbecuing with one of these, while ago I know it's not a chicken } \\
\text { rotisserie, but it certainly looks like one" }\end{array}$ & $\begin{array}{l}\text { A chicken } \\
\text { rotisserie }\end{array}$ & $\begin{array}{l}\text { The negative } \\
\text { space }\end{array}$ \\
\hline $\begin{array}{l}\text { PARTICIPANT LL: "It kind of reminds me of like you know when you go to a } \\
\text { restaurant and it's on your table, there could be, like a basket of stuff." }\end{array}$ & It's on your table & Presence \\
\hline
\end{tabular}




\section{Theme/exhibition topic C-TOOLS AND IDENTITY}

Another theme that emerged as a possible topic for an inclusive exhibit is centered on tools and their relationship with our identity. This theme emerged from categories that originated from in vivo codes that were critical about the juicer's design in the context of the blind lifestyle-the juicer was seen as something to look at, take up space, and not used (Box 3). Their impressions and experiences were categorized as personal identity, function (or lack of), and individuals who aspire to an identity. From those categories, the theme emerged whereby a tool's primary function is viewed as an extension of a person rather than as a device for performing a task.

From the categories and the theme, an exhibition topic was interpreted. The exhibition topic speaks to how the tools we use are an extension of ourselves, both as a part of our body but also a part of our identity. The Juicy Salif would provide context and evidence to the exhibition message as an example of an object that represents who we are and who we are not. 
Box 3

Example of thematic coding analysis leading to topic $C$

\section{EXCERPT}

IN VIVO CODES

CATEGORIES

PARTICIPANT ND: "Not a good purpose, I mean we know its purpose now, but it took us a while to figure it out so that's one thing. The other thing is

it's just not you know very blind friendly, even though there's no pointy parts or anything like that it's just, it's you know, it's going to make a mess and for us."

PARTICIPANT LL: "We already make mess. I only speak for myself. Why should I buy something that makes it more easy to make a mess? Why would I bother with that, I think?"

RESEARCHER: "Well, who do you think would own something like this, then, if it's not you."

PARTICIPANT LL: "People who don't really do it. They don't make juice but they think they might you know, like 'oh look at this fancy thing I have now', you know, like you know how people buy."

PARTICIPANT LL: "They're all cooks but they're not cooks so like they think they want to start cooking so they buy fancy stuff to do it it's not actually practical. I don't know. Like to me that it's almost like that, like someone who, yeah, they would just kind of think they might do it and it's on the counter and they go 'Oh look there's my nice new juicer thing' and then it just sits there or something or maybe if you had a bar. Like we're making drinks, maybe you would think 'fancy' when you know 'cuz you're not doing it to make like lemon loaf for you need like the juice of three lemons or something you know or something like that you want just a bit."

PARTICIPANT LS: "It is an inanimate artistic object, just for show. Just put it somewhere. Yeah, that's what it's for-show. That's its function."

PARTICIPANT DN: - "It looks nice. Sighted people like that kind of thing. You know, eye candy."

PARTICIPANT BN: "For those rich people, food just, it's always on the table at dinnertime, appears ..."

PARTICIPANT OT: "It kind of reminds me of you know, like, those, those era where wives were at home taking care of the kids ..."

Look at this fancy

thing I have

They're cooks but not cooks

They buy fancy

stuff

You're not [using] it to make lemon

loaf
Identity

Function

Identity

Identity

Aspiration of identity

Function is not

for cooking

Just for show

Eye candy [for

sighted people]

Food just appears

identity

Not for cooking

Reminds me of

Identity 


\subsubsection{Exhibition topics and the Contempra telephone}

This section provides an overview of themes and examples of exhibition topics drawn from the analysis of the participants' in vivo comments about the Contempra telephone. The comments were coded and categorized to reveal themes that were then interpreted into an exhibition topic that may offer a common point of access to a general visitor audience.

\section{Theme/exhibition topic A- HACKING}

This theme emerged somewhat differently than the previous three. For example, one of the initial codes, phone phreaking, was very significant and pointed directly to a theme of HACKING (Box 4). This initial code was evaluated again through subsequent reviews of the data, and other insights and experiences emerged as additional codes. Those new codes were interpreted into higher-order categories that were associated with phone phreaking such as community, blind identity, and suprasensory abilities. By interpreting the initial code that included phone phreaking into the theme of HACKING, as an exhibition topic, it could include other topics related to hacking such as repurposing old telephones to a table lamp, or popular culture that explored the identity and mystique of hacking such as the 1992 movie Sneakers.

Telephone hacking sometimes known as phone phreaking was one of the first instances of computer/digital hacking (Martin, 2007). The HACKING exhibit would display the Contempra telephone as a representation of this phenomenon that became popularized in the early 1970 s as telephony transitioned from analog to tone. The exhibition topic could include sub-themes related to the social/cultural impact of hacking as well as the technological mechanics of the phenomenon. 
Box 4

Example of thematic coding analysis leading to topic $A$

\section{EXCERPT}

PARTICIPANT DN: "If you want to go down a real rabbit hole, there was a phenomenon called phone phreaking, freak, like freak, phreaking. And ... blind people were highly represented in this group."

"But it was an early form of hacking of computer hacking. And so, it was totally accessible because it was all done over the phone."

"And so blind people were highly represented in this illegal movement, because it was all audio."

"It's not like I'm not saying blind people can't be computer hackers today. I'm sure we can. But that's partly a visual, you know, visual interface. But when it was just phone, when it was just the basic phone, blind people were arguably on an advantage because some of it was about being able to reproduce tones and hear subtleties."

"And so, the mechanics of how it worked, were really blind friendly."

"So, it's an early iteration of computer hacking that blind people were, you know, blind people who were totally geeky, had access to."

"Most people didn't even know about it at the time that blind people knew about. This is just stuff I heard from a friend ..."

"Um, yeah, so the super sophisticated people would hear the sound that was made by pressing the number four. So, those sounds are compilations of notes. So, when you press the number four, it's several notes. Yeah, but it's several notes put together. And if your ear is super cultivated, you can hear."

"Another example is that it was very memory based. So, if you happened to hack a code, like say, You kept trying and trying and trying and you got one, then you had to know what it was, you had to be able to remember it. Maybe you were writing them down."

"But so, memory and a sharp ear, I would say. Those are two things that that blind people have a bit of a trained advantage with. And so, the phone is such a simple interface."

"For some people in the blind community, it was access to the whole world in a certain sense."

"Anyway, it's a whole other side topic. I didn't mean to sidetrack but just when you talk about the emotional value of the phone, that's part of it for me was that, you know, if you somehow got involved in this subculture, it became access to a lot more than just calling your friend down the street."

\section{IN VIVO CODES}

Phone phreaking

Blind people

highly

represented

Totally accessible

Illegal movement

All audio

Computer hackers

today use a visual

interface

Blind people an

advantage

Blind friendly

Blind people geeky

People didn't

know about it

Super

sophisticated

people

If your ear is

super cultivated

You had to

remember

Memory and a

sharp ear

A trained

advantage

Access to the

world

Emotional value

of the phone

Subculture

More than just

calling friends
CATEGORIES

Hacking

Community

Suprasensory

Blind identity

Suprasensory

Blind identity

Community

Suprasensory

Community

Community

Identity

Suprasensory

Mundane

competency

Suprasensory

Mundane

competency

Community

Community

Identity 


\begin{tabular}{|c|c|c|}
\hline EXCERPT & IN VIVO CODES & CATEGORIES \\
\hline $\begin{array}{l}\text { PARTICIPANT SC: "This person figured out the frequencies of the of the } \\
\text { phone numbers so that he could record them on a Hammond organ or } \\
\text { something. And dial you know, called Russia." }\end{array}$ & $\begin{array}{l}\text { figured out } \\
\text { frequencies }\end{array}$ & Hacking \\
\hline $\begin{array}{l}\text { PARTICIPANT LL: "They were like these blind hackers that would whistle } \\
\text { the sounds of the tones into the phone and they would they would make } \\
\text { all these calls." }\end{array}$ & $\begin{array}{l}\text { whistle tones into } \\
\text { the phone }\end{array}$ & Hacking \\
\hline $\begin{array}{l}\text { PARTICIPANT NO: "But what I liked with these old phones, though, I was } \\
\text { able to take my parents' one apart when I was a kid and I was able to } \\
\text { wire my tape recorder to it, so, then I was able to record messages and I } \\
\text { was also able to play music into the phone and stuff like that. Yeah, lots of } \\
\text { fun, because once you popped off the phone cover it was all alligator clips } \\
\text { inside with screws, so it was easy just to connect. You know I figured out } \\
\text { which screw was for the audio and then I was free to do all sorts of cool } \\
\text { stuff that was fun." }\end{array}$ & $\begin{array}{l}\text { take my parents' } \\
\text { [telephone] apart }\end{array}$ & Hacking \\
\hline
\end{tabular}




\section{Theme/exhibition topic B-THE SPATIAL PRESENCE OF INFORMATION}

The theme-SPATIAL PRESENCE OF INFORMATION-emerged from a variety

of in vivo codes, but from two different participant perspectives. The first perspective centered on physical descriptions of the Contempra telephone that included codes about its smooth simplicity and featureless surface. For some, there was nothing mysterious about the surfaces because they were not screens. Other codes referred to the telephone's importance such as it had a special table and was associated with terrible news. Codes such as bulkiness and its singular purpose gave rise to categories such as reliability and comfort. The second perspective centered on the Contempra telephone as a segue or vehicle to discuss smartphones and communication technology today. The in vivo codes related to this second perspective, such as flashing and whizzing, and it follows us were compared to the codes related to the first perspective. From this analysis, higherorder categories such as active, passive, and access to information were used to group and re-examine the data. The result was a theme and exhibition topic about the presence of information, but from a spatial sense.

This theme emerged from looking at the categories within the context of the Contempra telephone and a smartphone and from the phenomenon of information push and pull. Both devices shared similar in vivo codes that highlighted their outward physical appearance when not in use-they both are featureless on the outside when not in use or when closed. However, unlike the smartphone, the Contempra appears restful when closed because it is a technology that pulls information. The smartphone is active and is doing things in the background. To the user, the smartphone's appearance was not interpreted as restful. Differences in the codes led to differences in categories. The Contempra's codes were categorized as passive and comforting which I attributed to its simple pull function and its closed, featureless appearance when not in use. This contrasts with the smartphone, a push device, with in vivo codes that I interpreted as 
active. Both collections of codes for the telephones, the Contempra, and the smartphone, led to the category, access to information.

The category labeled access to information can be connected to the phenomena of information push and pull that emerged in the communication industry in the early $1990 .^{29}$ It can be conceptualized as how users receive content from the Internet-they either 'pull' it on request, or they receive it as a 'push' where the content is delivered automatically. Digital content could then be imagined as having a physical form, or presence, by which devices, such as the Contempra and smartphone, deliver it and people receive it.

29 The push and pull of information is defined as the relationship between message traffic, user and electronic device, and the types of messages/information being sent (Cybenko \& Brewingtont, 1999). 
Box 5.1

Example of thematic coding analysis leading to topic B Contempra

\begin{tabular}{|c|c|c|}
\hline EXCERPT & IN VIVO CODES & CATEGORIES \\
\hline $\begin{array}{l}\text { PARTICIPANT OT: "It's kind of a comforting personality. Like, if you need a } \\
\text { phone, it's there for you to use." }\end{array}$ & $\begin{array}{l}\text { Comforting } \\
\text { personality }\end{array}$ & Comfort \\
\hline $\begin{array}{l}\text { PARTICIPANT EH: "... there was a place for the phone in your house like it } \\
\text { had a special table, your parents have a little table for that." }\end{array}$ & $\begin{array}{l}\text { Had a special } \\
\text { table }\end{array}$ & Importance \\
\hline $\begin{array}{l}\text { PARTICIPANT LL: "The phone was associated with like terrible news } \\
\text { sometimes, right?" }\end{array}$ & $\begin{array}{l}\text { Long distance } \\
\text { associated with } \\
\text { terrible news }\end{array}$ & $\begin{array}{l}\text { Access to } \\
\text { information }\end{array}$ \\
\hline $\begin{array}{l}\text { PARTICIPANT DN: "... with this it's more it's almost like a three- } \\
\text { dimensional kind of open box closed box. And so now I have access to } \\
\text { whatever I can connect to through the phone." } \\
\text { "The simplicity of it like there's any controls are sort of hidden inside so } \\
\text { that when the receiver is on the cradle, there's a really smooth } \\
\text { simplicity." } \\
\text { "There's nothing mysterious about this. It's not like I pick it up and think, } \\
\text { what's the display showing? Or what am I missing by not having } \\
\text { voiceover? I know that this is just what it appears to be. So that's part of } \\
\text { why it's restful. It is that the surface is just a surface. I like the aesthetic of } \\
\text { the smooth, almost featureless surface." }\end{array}$ & $\begin{array}{l}\begin{array}{l}\text { Three- } \\
\text { dimensional box }\end{array} \\
\text { Smooth simplicity } \\
\text { Nothing } \\
\text { mysterious } \\
\text { Surface is just a } \\
\text { surface } \\
\text { Featureless } \\
\text { surface }\end{array}$ & $\begin{array}{l}\text { Access to } \\
\text { information } \\
\text { Passive }\end{array}$ \\
\hline $\begin{array}{l}\text { PARTICIPANT SL: "The bulkiness to me implies that an object is very } \\
\text { reliable. It's not going to fail on you, especially if you drop it. So, to me, } \\
\text { bulky, implies reliability." }\end{array}$ & Reliability & Comfort \\
\hline
\end{tabular}


Box 5.2

Example of thematic coding analysis leading to topic B Smartphone

\begin{tabular}{|c|c|c|}
\hline EXCERPT & IN VIVO CODES & CATEGORIES \\
\hline $\begin{array}{l}\text { PARTICIPANT SL: "Now with the cell phone. It's with us. It follows us } \\
\text { wherever we are." }\end{array}$ & It follows us & $\begin{array}{l}\text { Access to } \\
\text { information }\end{array}$ \\
\hline $\begin{array}{l}\text { PARTICIPANT DN: "With the phone today, you flick it on and then the } \\
\text { whole world is projecting up at you." }\end{array}$ & $\begin{array}{l}\text { The whole world } \\
\text { projecting up. }\end{array}$ & Active \\
\hline PARTICIPANT NZ: "... whole phone is in your hand." & In your hand & $\begin{array}{l}\text { Access to } \\
\text { information }\end{array}$ \\
\hline $\begin{array}{l}\text { PARTICIPANT DN: "Also because I know that there's no screens. Like even } \\
\text { an iPhone, it is sort of restful in texture to because of its smooth surfaces. } \\
\text { But I know that it's flashing and whizzing with all sorts of moving visual } \\
\text { images. } \\
\text { "... my iPhone when it's off, it doesn't have that sense, because I know it's } \\
\text { still doing things in the background." }\end{array}$ & $\begin{array}{l}\text { Sensing flashing } \\
\text { and whizzing } \\
\text { iPhone is still } \\
\text { doing things in } \\
\text { the background }\end{array}$ & $\begin{array}{l}\text { Access to } \\
\text { information }\end{array}$ \\
\hline $\begin{array}{l}\text { PARTICIPANT LL: "Now you know, like, I have a feeling I could be wrong, } \\
\text { but the people are gonna say 'Oh, I only want my phone just to be a } \\
\text { phone'." }\end{array}$ & $\begin{array}{l}\text { A phone just as a } \\
\text { phone }\end{array}$ & $\begin{array}{l}\text { Access to } \\
\text { information } \\
\text { Passive }\end{array}$ \\
\hline
\end{tabular}




\section{Theme/exhibition topic C-MUSCLE MEMORY}

The theme for the exhibition topic-MUSCLE MEMORY-emerged from the participants' comments when the Contempra telephone was used as a cultural probe to discuss other topics. The initial codes that emerged from the transcripts originated from personal recollections about calling friends on the phone, demonstrations on how to use a rotary telephone when blind, and descriptions of how a credit card number is memorized. The codes, such as my best friend's number and remembering the shape of a number, were later re-examined and organized into categories which included deep memory, action, muscle movement, training, and spatial memory. From those categories, the theme of MUSCLE MEMORY was defined and broadened into an inclusive narrative that could include muscle memory in everyday life such as music and sports.

The exhibit's message communicates how muscle memory plays a role in our everyday lives by creating maximum efficiency within our system to complete a task. The exhibit defines muscle memory as a form of memory that is created through the repetition of a specific motor task such as dialing a telephone number. The process decreases the need for attention and creates maximum efficiency between motor and memory systems. Through tactile and audio-based interactives, the exhibit provides shared learning experiences of how muscle memory impacts our everyday lives, for example, in the experience of sports, music, walking to the bathroom in the dark, or tying your shoelaces.

The Contempra telephone exhibit would illustrate the concept of muscle memory by demonstrating how muscles and memory behave differently when using two different user interfaces-the rotary dial versus the touchpad dial. With all other variables the same, including the telephone model and number, the active learning exercise demonstrates how a different type of muscle memory is engaged with each interface. 
Box 6

Example of thematic coding analysis leading to topic C

EXCERPT

PARTICIPANT OT: "I actually dialed my very first, well, one of my first

phone numbers. This one was the one that I had since I was eight years old. It was 7636778."

PARTICIPANT OT: "I dialed my first best friend's number."

PARTICIPANT SL: "When you are going to make a call you have to count and what if you miss a hole? What if you miss count, and you're and you put your finger in the thing and you turn it and you thinking you're turning the six when you really ended up turning the five?"

PARTICIPANT EH: "I used my first push button phone in 1967. And I remember that because I was at Expo 67 in Montreal. And they were just bringing out the touchtone phones and they had at the bell pavilion, in Montreal at Expo 67. They were trialing the push button phones. And I didn't understand, I didn't know where how it worked. Like, I didn't know how the button layout went. But I knew I liked it a lot better than the, than the rotary dial."

PARTICIPANT DN: "And it's so manual to turn the dial there's a somatic quality where your body has to do I mean, we tap on a phone and swipe. So that's it, it's just a different set of, of gestures to make things work. But with this, the dialing process is manual. And so, you're, I don't know your body is engaged in a slightly different, slightly different way."

PARTICIPANT DN: "And I feel like phone numbers were an amazing early training and memory. Because you had either, you know, seven digits or eventually 10 digits, and I was always, you know, the one who remembered everyone's phone number and it's partly because it's by training. I think that it's not that easy to just unsure you know, [TX] to you can't judge? Oh, what's your phone number? It's just okay, jotting it down. It's you know, you don't always have a slate and stylus or Brailler."

"And so you cultivate your memory."

"Very early on phone numbers were a huge part of building my memory skills, huge part."

PARTICIPANT DN: "When I memorize any set of numbers, let's say I'm memorizing my bank card number or my MasterCard number, my mnemonic is to imagine the keyboard of a telephone - of a push button phone. So that's the way you do that too [TX]?"

PARTICIPANT TX: "Yes, I do the same thing here. I know my OHIP number, my SIN number, or the number you tell me. I know it."

\begin{tabular}{|l|l|}
\hline IN VIVO CODES & CATEGORIES \\
$\begin{array}{l}\text { My first phone } \\
\text { number }\end{array}$ & Deep memory \\
\hline $\begin{array}{l}\text { My best friend's } \\
\text { number }\end{array}$ & Deep memory \\
\hline $\begin{array}{l}\text { To make a call } \\
\text { you have to count }\end{array}$ & Action \\
$\begin{array}{l}\text { I know my } \\
\text { imagine keyboard }\end{array}$ & Training \\
$\begin{array}{l}\text { Yuilding memory } \\
\text { skills }\end{array}$ & Training \\
$\begin{array}{l}\text { I didn't know how } \\
\text { it worked. } \\
\text { But I knew I liked } \\
\text { it }\end{array}$ & Ease of use \\
\hline $\begin{array}{l}\text { Manual to turn } \\
\text { the dial } \\
\text { Tap and swipe }\end{array}$ & $\begin{array}{l}\text { Muscle } \\
\text { movement }\end{array}$ \\
\hline Ease of use \\
\hline
\end{tabular}




\begin{tabular}{|c|c|c|}
\hline EXCERPT & IN VIVO CODES & CATEGORIES \\
\hline $\begin{array}{l}\text { PARTICIPANT DN: "Me too. Me too. And the way I remember is like it's } \\
\text { spatial. There's something spatial in my head that uses the telephone } \\
\text { keypad and it's early training, I'm sure it's just that that's what I grew up } \\
\text { with." } \\
\text { "And my mind's learned to memorize numbers in that way. So yeah, I've } \\
\text { got, like [TX] and like many blind people, I've got a lot of numbers in my } \\
\text { memory and that's how I store it and retrieve it. It is that configuration of } \\
\text { the twelve-key keypad that's on computers. But my visualization, my } \\
\text { spatial representation in my mind, is definitely the phone. It's based on } \\
\text { the phone." } \\
\text { PARTICIPANT TX: "And sometimes you can't get it. So you go to a phone, } \\
\text { and then you remember all of a sudden." } \\
\text { PARTICIPANT DN: "There's something in my fingers too. I feel my fingers } \\
\text { kind of want to wiggle a little bit to help me." }\end{array}$ & $\begin{array}{l}\text { I remember it's } \\
\text { spatial } \\
\text { Uses the } \\
\text { telephone keypad } \\
\text { Spatial } \\
\text { representation in } \\
\text { my mind is the } \\
\text { phone } \\
\text { Remembering all } \\
\text { of a sudden } \\
\text { Fingers wiggle to } \\
\text { help }\end{array}$ & $\begin{array}{l}\text { Spatial memory } \\
\text { Deep memory } \\
\text { Muscle } \\
\text { movement }\end{array}$ \\
\hline $\begin{array}{l}\text { PARTICIPANT LL: "When I would dial them, I would remember like, } \\
\text { especially on the touch tone like this shape of that number ... you } \\
\text { remember the shape." }\end{array}$ & $\begin{array}{l}\text { Remember the } \\
\text { shape of the } \\
\text { number }\end{array}$ & Spatial memory \\
\hline
\end{tabular}




\subsubsection{Exhibition framework and proposed exhibit module}

From the six different themes/exhibition topics that were drawn from the analysis, only one was synthesized into the proposed exhibit module. The remaining five were presented to demonstrate the variety of inclusive messages that emerged from the data and the potential of this approach to support an accessible exhibition development strategy.

This section compares an ocularcentric and a non-ocularcentric message for an exhibit of the Contempra telephone. This comparison provides an examination of how an identical object can be displayed in an exhibit that may rely on sensory accommodations for visual loss versus an exhibit with integrated accessibility based on a participatory object reading approach. The ocularcentric narrative for the Cool ' 6 os exhibit at the Canadian Museum of History was centered in Canadian identity, the International Style, post-war modernism, and the efficiency of materials and manufacturing processes. The chosen theme/exhibition topic with a non-ocularcentric narrative for the comparison was MUSCLE MEMORY.

The narrative for the proposed MUSCLE MEMORY exhibit is centered on the role of muscle memory in our everyday lives. As a result of its proposed inclusive narrative, accessibility may be better integrated into the delivery of the muscle memory content compared to the approach taken in the Cool ' 60 's ocularcentric exhibit. The theme/exhibition topic of MUSCLE MEMORY was chosen because of its rich data for a very inclusive message. The audio format of the handset and push-button interface of the telephone would act as a tool to deliver, organize, and choose content contributing to the overall MUSCLE MEMORY message. It would also be naturally accessible to visitors with vision loss. The perspectives and stories gathered in the object research sessions provided ample data to inspire active learning opportunities such as quick dialing challenges or remembering a number by finger movements. The other themes/exhibition 
topics that emerged from the thematic analysis also shared inclusive attributes, for the purposes of this thesis, the MUSCLE MEMORY topic was chosen because it would best serve to answer the research question 'How might we improve access to museum exhibitions through the exploration of inclusive narratives?'.

Table 1 compares the exhibition topics, Cool 6os and MUSCLE MEMORY. The comparison looks at examples of the wording of the object's physical and aesthetic characteristics in an extended object label. Examples of audio-tactile interactions that contribute to the understanding of the exhibit topic are also described in the framework.

Table 1

Exhibition framework comparing the Cool '6os exhibit to the proposed MUSCLE MEMORY exhibit

\begin{tabular}{|c|c|c|}
\hline \multirow[t]{2}{*}{$\begin{array}{l}\text { EXHIBIT } \\
\text { COMPONENT }\end{array}$} & $\begin{array}{l}\text { SELECTED ELEMENTS FROM AN } \\
\text { OCULARCENTRIC NARRATIVE }\end{array}$ & $\begin{array}{l}\text { INCLUSION AND INTEGRATED } \\
\text { ACCESSIBILITY }\end{array}$ \\
\hline & CoOL ‘60S & MUSCLE MEMORY \\
\hline \multirow[t]{2}{*}{$\begin{array}{l}\text { EXHIBIT } \\
\text { NARRATIVE }\end{array}$} & $\begin{array}{l}\text { "This exhibition celebrates Canadian } \\
\text { industrial and artistic design of the 1960s, } \\
\text { a vibrant and turbulent decade that } \\
\text { brought enormous change to Canada and } \\
\text { to Canadian-made products. Led by the } \\
\text { federal government, Canada wanted to } \\
\text { show the world that it was now a modern } \\
\text { and innovative country, firmly focused on } \\
\text { the future. It was a heady combination for } \\
\text { Canadian artists and designers." } \\
\text { (Canadian Architect, 2004) }\end{array}$ & $\begin{array}{l}\text { How muscle memory plays a role in our } \\
\text { everyday lives by creating maximum } \\
\text { efficiency within our system to complete a } \\
\text { task. }\end{array}$ \\
\hline & $\begin{array}{l}\text { Contempra telephone artifact label: } \\
\text { "With its sculptural form and availability in } \\
\text { a wide range of colours, the Contempra } \\
\text { phone was positioned for consumers as a } \\
\text { fun - rather than strictly utilitarian - } \\
\text { object. Designed and produced in Canada, } \\
\text { it was exported worldwide for many } \\
\text { years." (Canadian Museum of History) }\end{array}$ & $\begin{array}{l}\text { Example of an integrated accessible } \\
\text { artifact label: } \\
\text { The Contempra telephone is an example of } \\
\text { dials evolving from analog rotary to digital } \\
\text { touchpad and requiring a new set of } \\
\text { memory skill to input numbers. } \\
\text { Its long and streamlined form, about the } \\
\text { size of a paperback book was notably } \\
\text { heavier compared to today's telephones. A } \\
\text { shiny yet featureless exterior made it } \\
\text { appear restful on the tables or walls of } \\
\text { many family homes and offices. }\end{array}$ \\
\hline
\end{tabular}




\begin{tabular}{|c|c|c|}
\hline \multirow[t]{2}{*}{$\begin{array}{l}\text { EXHIBIT } \\
\text { COMPONENT }\end{array}$} & $\begin{array}{l}\text { SELECTED ELEMENTS FROM AN } \\
\text { OCULARCENTRIC NARRATIVE }\end{array}$ & $\begin{array}{l}\text { INCLUSION AND INTEGRATED } \\
\text { ACCESSIBILITY }\end{array}$ \\
\hline & COOL ‘60S & MUSCLE MEMORY \\
\hline $\begin{array}{l}\text { TACTILE AND } \\
\text { AUDIO } \\
\text { INTERPRETATION } \\
\text { AND } \\
\text { INTERACTIVITY }\end{array}$ & $\begin{array}{l}\text { The telephone was displayed inside a } \\
\text { sealed Q-bit system (Hunchuck et al., } \\
\text { 2006). }{ }^{30} \\
\text { An interactive Q\&A flip panel accompanied } \\
\text { the exhibit and notes in the Interpretive } \\
\text { Plan suggest the exhibition had planned } \\
\text { accessible components, for example, tactile } \\
\text { labels (A. Walpole, personal } \\
\text { communication, July 27, 2021). }\end{array}$ & $\begin{array}{l}\text { A reproduction of the telephone would } \\
\text { have the following interactive functions for } \\
\text { the visitor: } \\
\text { - visitors should be able to bring the } \\
\text { handset to their ear; } \\
\text { - the rotary dial should spin and create } \\
\text { the same sounds and vibration; } \\
\text { - the touch pad button dial should make } \\
\text { tones; and } \\
\text { the weight of the handset should be } \\
\text { accurate. }\end{array}$ \\
\hline EXHIBIT DESIGN & $\begin{array}{l}\text { Figure } 20 \text { shows how the exhibition design } \\
\text { "aestheticizes" (Hunchuck et al., 2006, p. } \\
\text { 84) the telephone by containing it within } \\
\text { the system of steel and glass panels. It was } \\
\text { mounted in the closed position on a plinth } \\
\text { adjacent to a side chair. }\end{array}$ & $\begin{array}{l}\text { The Contempra telephone object would be } \\
\text { reproduced and integrated into the exhibit } \\
\text { design as the primary conduit for delivering } \\
\text { the content via the audio on the } \\
\text { telephone's handset. } \\
\text { The audio on the handset would be } \\
\text { supplemented with directional speakers } \\
\text { allowing the visitor group to share the } \\
\text { exhibition experience. } \\
\text { Appendix D has a full description of the } \\
\text { exhibition design including components } \\
\text { related to visitor experience. }\end{array}$ \\
\hline
\end{tabular}

30 The Q-bit system is a modular exhibition display system that uses a steel framework and glass or solid panel walls that fit into a $3 \mathrm{D}$ cube grid (Hunchuck et al., 2006). Figure 20 shows the Q-bit system as used in the Cool '6os exhibition. 
The following is a description of the exhibit design for the proposed MUSCLE MEMORY exhibit module. The components in the proposed exhibit module applied comments that emerged from the participants in the object reading sessions. It is an example of how a theme that emerged from the study's thematic analysis would incorporate participant's insights into the exhibit design. The other themes that emerged discussed above could be examined in the same manner. This description was used as part of the script for the round-table discussion where the exhibit module was presented to the participant group for their evaluation.

\section{Box 7 MUSCLE MEMORY Description}

Encountering the exhibit as a visitor
The following is an exhibit visitor experience scenario that uses some of your comments as inspiration.

- Imagine walking into a museum with a group of friends or with family. You are beckoned toward an exhibit (via a proximity sensor) by an audio message. This audio message invites you to visit the Muscle Memory exhibit. The message encourages you "to learn how muscle memory plays a role in your everyday life as you interact with the objects around you and the tasks you do".

- As you approach the exhibit, you can hear a traditional telephone ring and a message inviting you to "Answer the telephone". When you lift the handset, you hear a voice telling you about the exhibit that you are about to enter. This voice is also heard through a directed speaker for others to hear.

- The 'voice' gives you some instructions about how to interact with the exhibit. It tells you that there are a series of handsets to be lifted to hear more about the topic.

- Each subsequent handset has a different method of 'dialing'. The audio from the handset tells you about the relationship between your muscles, your memory, and the repetitive tasks you do every day.

- You are free to lift any of the handsets in any order to learn different things about the exhibit topic. You would be able to select what interests you by pressing a button on the keypad much like when you call a bank.

- Interactive activities for the exhibit will challenge your different muscle memory skills as part of active learning. One activity would ask you to dial the same telephone number into different types of dialing interfaces - rotary, touchtone, and computer keyboard. The sound from the different dials could be important too. You could also be challenged on how fast you would be able to input a number without making a mistake. The challenge could be reminiscent of dialing into a radio station contest you could compete against for friends or other visitors.

- $\quad$ The audio you hear from the handset also acts as object labels and provides you with an integrated audio description about the Contempra telephone. It describes how the 


\begin{tabular}{|c|c|}
\hline & $\begin{array}{l}\text { telephone was designed and produced at a time when the input interfaces were } \\
\text { changing because of changing technology. } \\
\text { - The audio description of the Contempra telephone describes it as: } \\
\text { "The Contempra telephone is an example of dials evolving from analog rotary to } \\
\text { digital touchpad and requiring a new set of memory tools to input numbers." } \\
\text { "It's long and streamlined form, about the size of a paperback book was notably } \\
\text { heavier compared to today's telephones. A shiny yet featureless exterior made } \\
\text { it appear restful on the tables or walls of many family homes and offices." }\end{array}$ \\
\hline $\begin{array}{l}\text { The entire exhibit } \\
\text { would be audio and } \\
\text { tactile based }\end{array}$ & $\begin{array}{l}\text { - The exhibit uses audio to deliver the content. The audio would come from the } \\
\text { handset and could simultaneously be heard through a directional speaker allowing } \\
\text { your entire group to hear. } \\
\text { - The tactility of the handsets and the subsequent variety of dialing interfaces would } \\
\text { be as accurate as possible to reinforce the relationship between muscles, repetitive } \\
\text { tasks, and memory. } \\
\text { - The handset, with its angled shape, would allow you to hold it 'hands-free' on your } \\
\text { shoulder. } \\
\text { The audio feedback from the handsets in the form of clicks from the rotary dial or } \\
\text { tones from the touch pad are also used to reinforce the message of memory and } \\
\text { repetitive tasks - visitors would be asked to input numbers on a touchtone phone and } \\
\text { hear a tune - they could then be asked to reproduce that tune by dialing on the pad } \\
\text { again - but this time from memory. Suggestions will be given on how to remember } \\
\text { numbers using a touch pad by focusing on the 'shape' of the number or the } \\
\text { movement of your fingers. } \\
\text { Deep dives into the exhibit content could talk about phone numbers that have been } \\
\text { burned into your memory like that of your best friend, } 911 \text { emergency, or telephone } \\
\text { numbers used as an advertising device. } \\
\text { You would be invited to share a memorable number and/or story about it by } \\
\text { recording yourself through a telephone connected to an 'answering machine'. Your } \\
\text { recording would be saved for other visitors to hear and as an audio artifact for the } \\
\text { museum collection. } \\
\text { depending on the section you are in or indicate the presence of an object. }\end{array}$ \\
\hline
\end{tabular}

\subsubsection{Round-table discussion}

From the round-table discussion conducted with some of the object research session participants, two types of findings emerged. The first type centered on the exhibit module's inclusivity, accessibility, and visitor experience. The participants compared the ocularcentric and a non-ocularcentric exhibits that displayed the Contempra telephone 
(Table 1). What emerged from this discussion was the common perspective that they found it interesting that an exhibit topic came from their comments in the object research sessions. Members of the group discussed the social aspects of the proposed visitor experience in the module as positive, stating that they prefer to visit museums with friends. The findings that emerged from a lengthy discussion about inclusive narratives and accessible exhibition content delivery practices revealed their sensitivity to 'othering' and their extensive opinions about different accessibility accommodation techniques (their preferred accessibility accommodation is phoning ahead and asking for a human guided tour). However, at the same time, a comment that the inclusive narrative should include a sub-theme about non-visual muscle memory suggests the importance of their blind identity and to communicate 'being different but the same' as part of a narrative. In the case of the MUSCLE MEMORY exhibit module, participants were pleased with this exhibit's inclusive narrative and that the design did not create an 'othering' environment.

The second type of finding emerged from questions that asked them to evaluate the object research sessions, in particular the focus group method and their distinct sensory abilities. They commented on how the compatible pairings allowed them to "bounce ideas back and forth" (Participant ND). When asked how their sensory abilities contributed to object research, their responses were few. However, at one point the participants connected my questions about their research experience and contribution related to sensory abilities, MUSCLE MEMORY, and the small size of the blind community by bringing forth a short remembrance to the late Jeff Healey, an accomplished guitarist with vision loss whom they all knew of and respected. "He [Jeff Healey] would sit after school, outside ... and play guitar at 8 [years old]." (Participant LL). These comments that originated from the discussion about the MUSCLE MEMORY 
topic could easily be added to the categories of identity, suprasensory, and community that emerged in the thematic analysis for the exhibition topic, HACKING.

\subsection{Summary of findings}

The study's findings provide a step forward to improving access to museum services for visitors with vision loss. The strategy set forth to minimize barriers and improve access through inclusive narratives was conducted through an object research session with people who are blind using qualitative research methods. The study produced a series of findings based on three main areas: 1) findings which led to the themes/exhibition topics; 2) findings related to the design of remote object reading sessions; and 3) factors to consider when working with people who are blind to conduct remote research.

This study led to findings to support:

- the development of 6 distinct themes/exhibition topics grounded in the narratives of participants who are blind;

- the development of a more extensive exhibit module to explore these findings;

- the comparison of the proposed exhibit module to an existing ocularcentric exhibit;

- findings related to the design of remote object reading sessions, and in particular, working with the blind community; and

- factors to consider when working with people who are blind to develop exhibits.

The narratives proposed from the findings for themes/exhibition topics were inclusive and with integrated accessibility in the exhibit design components. For example, the narrative for the theme of VICTORIAN ERA AESTHETIC included categories of craftsmanship, ornament, and status as characteristics of objects that embody that era. These characteristics can easily translate into a non-visual description. 
The findings that emerged from my experience in conducting remote research are directly related to collaborating with individuals with vision loss. For example, in conducting remote object readings, I was able to recruit a group of individuals from different regions across Canada, rather than restricting the activity to local [Ottawa] participants. ${ }^{31}$ The findings revealed nuances distinct to the participant group that point to the importance of understanding subtle contextual considerations in designing remote research. For example, the time-of-day when recordings took place influenced the quality of the video footage since the lights were not on later in the day to illuminate the setting. Further, there is a need for redundancies or back-up plans in the research design to compensate for inaccessible courier delivery practices. In the object reading sessions, the technique and tradition of using nitrile gloves created a barrier to participants' ability to draw from their sense of touch to inform their interpretations and our discussion. Participants' pre-existing relationships with each other were a concern for some participants due to the small community of participants with congenital and early-onset blindness. Although I did not set out to 'test designs', but rather, consult with real people about the opportunities and possibilities for inclusive exhibit narratives, the participants' involvement in prior research studies that focused on 'product testing' may have primed their expectations about and interactions in this study. Finally, from the round-table discussion, the findings included the group's emphasis on the narrative of 'identity', of being 'different but the same', as important to communicate and their custom of phoning ahead to learn what type of accessibility accommodations a museum uses as part of their decision-making process when considering a visit to a museum.

${ }^{31}$ Although a list of the regions in Canada from where the participants were recruited may help to demonstrate how remote research allowed for a broader reach, it was not published to prevent the possibility of identifying participants. 


\section{Discussion}

The findings from the study suggest that improving access to museum exhibitions through inclusive narratives for people with vision loss benefits from a strategy that uses interdisciplinary practices, qualitative methods, and collaboration with people with congenital and early-onset blindness. In this study, interdisciplinary practices, in the form of object research sessions, included object research and the use of cultural probes, to explore narratives that may arise from participants' distinct sensory abilities and mental models. Through 2-person interviews in a focus group setting, two 'museumtype' objects-the Juicy Salif and Contempra telephone-were discussed with participants. I was able to code the narratives of participants using in vivo coding and develop a thematic analysis from this work. From this analysis, non-ocularcentric object narratives, inclusive themes/exhibition topics, and accessible object descriptions emerged. They were synthesized into a proposed exhibit module that served to address the complexity of accessible museum exhibitions and demonstrate an inclusive exhibition topic to the participant group inspired and generated from their stories. The findings suggest that this strategy could improve access through inclusive narratives for visitors with vision loss. These findings build on and intersect with previous research that explored accessible exhibition development, multisensory object research, and objects as cultural probes.

Other findings that emerged from this study relate to conducting remote research with the blind community. Important factors to consider in designing remote research with this participant group may not necessarily be obvious to researchers with sight. These factors include labeling courier packages and their contents with Braille, providing instructions to couriers with the exact details of where to leave a package, following up on its delivery, and realizing that some participants may require a lamp if the time-ofday for recording is at night. These experiences in designing research and how the design 
is received and experienced by participants contribute to a body of knowledge that could be useful when planning future research designs that involve remote research.

\subsection{Findings and round-table discussion}

The round-table discussion led to findings about the inclusivity of the exhibit module, its comparison with an ocularcentric exhibition with the same object, and an evaluation of the object research sessions from the participant's point of view. The findings suggest that participants were unaccustomed to inclusive museum exhibits for people with vision loss. Comments on how they were intrigued that an exhibit topic was derived from their sessions' statements were unexpected as were their comments that the exhibit could now allow their visitor experience to be shared with friends. Participants were also surprised that the proposed exhibit module would not, in theory, require an accessibility accommodation such as audio guides, a separate room for blind visitors, or a human tour guide. This suggests participating in exhibition development research may be a novelty that could benefit from further exploration. Another finding from the round-table discussion is related to the participants' reluctance to attribute their sensory abilities and insights to advances in material culture studies research or the development of inclusive exhibitions (not just accessible exhibitions). This may be evidence to support Reyes-Cruz et al (2020) statements that people with vision loss consider their competencies as unremarkable.

\subsection{Findings and previous work}

Together, the findings suggest that object research sessions, which have been discussed in previous literature (Howes, 2014; Krmpotich, 2020; Mills, 2018; Peers \& Krmpotich, 2014; Woodward, 2016) and processes for accessible exhibition development (Cachia, 2019; Chick, 2018; Hesseldahl et al., 2018; Laurentino et al., 2021; Osborne et 
al., 2014; Shimamura-Willcocks, 2011) may lead to findings that can help form the strategy to improve access through inclusive narratives for visitors with vision loss (Figure 30).

Figure 30

This study relative to previous related literature

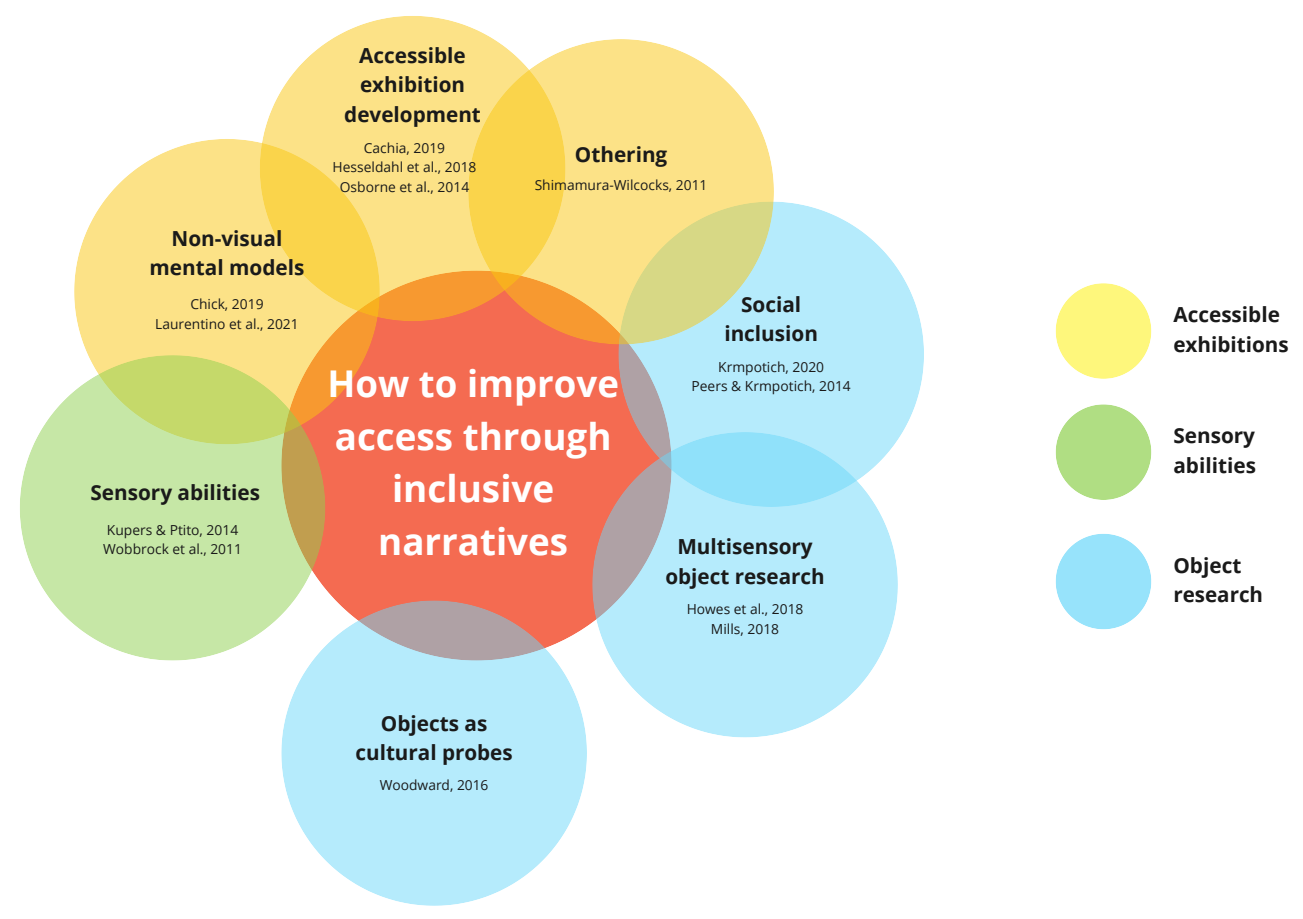

Note: This image illustrates how the study and research question are positioned relative to the literature and at the intersection of accessible exhibition development, multisensory object research, cultural probes, social inclusion, and sensory abilities. The different areas of literature have been arranged to demonstrate how they intersect with each other and my study.

\subsubsection{Accessible exhibition development}

Broadly, the findings from the study support previous literature that recommends accessibility be considered early in the exhibition development process (Cachia, 2019; Hesseldahl et al., 2018; Osborne et al., 2014) and that people with visual loss can benefit from exhibition messages interpreted using their sensory abilities as a starting point (Chick, 2018; Laurentino et al., 2021; Shimamura-Willcocks, 2011). These findings also contribute to the theory that interdisciplinary practices can begin to tame 
some of the complexity that may be encountered later on in the process when developing accessible exhibitions (Moreno \& Villalba, 2018).

By design, at the outset of exhibition development, the inclusive narrative and accessible exhibition topic emerges from the object research sessions. This is because individuals with congenital and early-onset blindness are part of the object research sessions, and the experiential and sensory barriers can be minimized since the exhibition topic originates from non-ocularcentric data. What is interesting is that this finding also speaks to the nature of the complexity discussed in Tesler's law of the conservation of complexity, where the complexity of a system is constant and by simplifying one part of the system, the complexity is not removed but rather shifted to another part (Norman, 2010). In this case, the complexity of the accessible exhibition system is shifted away from the visitor's experience to the outset of exhibition development. At the beginning of the exhibition development process, solutions for inclusivity can emerge from collaboration with the community, and accessibility can be addressed upfront, whereby the complexity is borne by the museum exhibition development team.

\subsubsection{Objects as cultural probes}

The findings related to the study's participatory design approach and qualitative analysis build on Woodward's (2016) work, where they used Gaver's (1999) technique of cultural probes to engage participants in a dialogue about a personal object. Woodward's research was centered on interdisciplinary methods when collecting, analyzing, and interpreting data in material and material culture studies. Woodward's study concluded by highlighting the participants' insights about the wear and tear of an object and speculations on how it reflects the identity of the previous owner. With those insights, an artist synthesized, from the original object, a new object whose purpose was to highlight the participants' insights. 
My study was similar to Woodward's in that it also collected data using objects as cultural probes, interpreted participant comments through thematic coding analysis, and then synthesized findings. However, my study included the participation of people with congenital and early-onset blindness and 'museum-type' objects. Another difference was an object in my study was synthesized into an exhibit module for a museum exhibition. My findings suggest that the added dimension of non-visual insights into Woodward's methodology has the possibility to generate inclusive object narratives inspired from participants' stories for synthesis into an accessible exhibit.

The positive comments about the exhibit module from my research participants in the round-table discussion, who compared it with a baseline ocularcentric exhibit that displayed the same object, provide evidence to support participatory design practices (in particular the use of cultural probes) in the exhibition development process for inclusive and accessible exhibitions.

\subsubsection{Multisensory object research}

This study supports Howes et al. (2018), Mills (2018), and Krmpotich's (2020) findings that attribute multisensory and inclusive object examination to richer interpretations in material culture studies and the idea that suprasensory abilities go beyond abilities in sensory discrimination to include the life experiences and mental models of the world created by the perspectives that these abilities afford (Catteneo \& Vecchi, 2011). In my study, the findings suggest that people who are blind can contribute to research in material culture studies by adding another dimension to multisensory object examination that ocularcentric researchers may not be able to relate to or appropriate. The findings support the idea that recognizing competencies in sensory abilities is a new point of entry, offers alternative interpretations of objects resulting from participants' differing sensitivities to sound, touch, vibration, and spatial perception. For example, the thematic analysis of the Contempra codes-infused with 
participants' language about communicating with friends, telephony, and the mechanics of dialing-led to higher-order categories emphasizing the importance of community, blind identity, suprasensory abilities, and hacking in the narratives. These perspectives about the telephones may not have emerged through ocularcentric-based research. However, this remains to be studied.

Integrating the distinct sensory abilities of participants can possibly lead to other research, for example, non-visual multisensory examination practices. Such studies can contribute to a deeper understanding of an object's form, surface relief, materials, weight, and temperature. The benefits of non-ocularcentric examination of objects in material culture studies versus the examination of objects by sighted researchers could lead to new insights and narratives. The findings can perhaps be used to compare the value of new knowledge gained through non-ocularcentric examination practices versus the value of object preservation and conservation (Saunders, 2014). This comparison can contribute to the literature about the management of cultural heritage that suggests an object must have some other purpose beyond being conserved. In turn, the findings may also challenge the tradition of ocularcentric values (Classen \& Howes, 2006) and the residual mid-nineteenth century practice of controlled access to museum collections for “experts” (Peers \& Krmpotich, 2014, p. 37).

\subsection{Remote research experience}

This study's findings are in line with previous literature that identified the benefits of remote research. The benefits included a reduced operation cost, access to a larger pool of geographically dispersed participants, and, perhaps as a result, better compatibility of focus group pairings for participant rapport (Archibald et al., 2019; Topping et al., 2021). These benefits are especially pertinent to researchers planning on studies that involve participants with congenital and early-onset blindness. The findings 
support the idea that remote research over face-to-face interviews reduces the likelihood of limitations caused by sample size and can allow for more diverse participants compared to a small local participant population (Abrams et al., 2015). Other findings that emerged from conducting remote research that may be important in remote research planning included issues with the delivery practices of courier services and the time-of-day for recording. The documentation of conducting and participating in this remote research study are consistent with previous literature that speaks to the advantages and disadvantages of remote research and the nuances that may affect the study design, participant experience, and data collection (Abrams et al., 2015; Archibald et al., 2019; Topping et al., 2021).

\subsection{Limitations}

\subsubsection{Participant age}

There is a possibility that the age range of participants may have created a limitation that affected the data. During the recruitment phase there was a lack of participants in the 18 to 40 years age range who responded to the invitation to participate. This lack of response may be due to a demographic shift in the 18- to 40year-old demographic with a rarity of congenital blindness-one of the participant inclusion criteria. The shift is due to an advancement in paediatric medicine over the past fifty years that minimized retinopathy of prematurity, a frequent cause of congenital blindness (Shah, 2016). The age bias may have influenced the collection of stories that were based on life experiences. An example would be the story about using a rotary dial to call into a radio station's contest. An 18- to 40-year-old demographic may have had a different story, which in turn, may have led to different themes/exhibition topics. Although my study has an age bias, the findings do not suggest that a younger 
demographic would have affected the ability for inclusive narratives to emerge using thematic analysis.

In spite of this limitation, the exploratory nature of my study may have benefited in other ways from the participants' similar age demographic. For example, their similar life experiences allowed the in vivo codes to be more focused and the emerging themes from the categories stronger.

\subsubsection{Participants' prior research experience}

Participants' previous experience as product testers may have influenced their responses in object research sessions. Some participants with experience in conducting product testing may have been unaccustomed to responding to questions about object examination and description. The responses from experienced participants were more focused on the operation of the objects, which could have influenced the findings.

\subsubsection{Data collection due to remote research}

In this study, remote research leverages its potential in geographic reach and uses an internet-based video-conferencing platform, Zoom. However, this practice may not be practical to museums and may also exclude certain populations. There may have been an advantage of remote research to recruit a more diverse sample size since participants do not need to be compensated for travel costs. This potential advantage in conducting remote research in this study would be consistent with previous literature (Archibald et al., 2019; Reid \& Reid, 2005; Topping et al., 2021). However, the benefits of remote research that my study was able to leverage may not exist in practice. For example, when working with authentic museum objects, it may not be practical to use remote research practices.

During this research, a standard commercial courier service was used to ship the objects to participants. In reality, examining some museum objects, such as artifacts, 
require specialty shipping containers and museum professionals (such as curators, preparators, and collection managers) to deliver, chaperone, and protect the objects. Another limitation of remote research is the cost of Internet access and computer equipment. This cost would be either borne by the museum or by the participants. Participants who could not bear the cost would be excluded from the study (as in my study where access to the Internet and familiarity with the Zoom application were part of the inclusion criteria). Similar to the age bias of participants mentioned in Section 5.4.1, this limitation can lead to an unrepresented population that can affect the data (Abrams et al., 2015; Topping et al., 2021).

It is important to bear in mind that while some museum practices may not be able to benefit from the advantages of remote research, the limitations mentioned above are hypothetical and each study's context would need to be examined to understand the merits of conducting this approach.

A final finding in this study that is consistent with previous literature suggests how remote research can create another limitation through a reduced supervision of the participant. Premature access to the research kit by participants or their companion(s) with sight may influence their comments about their 'first impressions' in object research sessions. This finding corroborates previous literature that identifies how the external environment surrounding the participant may be problematic (Topping et al., 2021). However, this limitation can be easily compensated for in the design of the research kit and the directions given to participants.

\subsection{Further research}

There is abundant room for further study. Duplicating the object research sessions and thematic analysis with different objects or priming participants about their role as examiners could appraise the viability of the strategy to develop inclusive 
narratives for people with vision loss. Future work could involve developing a fuller examination of the exhibit module through a prototype that could be physically evaluated to provide qualitative results that evaluate visitor experience, and more specifically, experiential and social barriers. And finally, alternative approaches could explore whether different findings emerge with a more diverse participant group examining objects.

Broadly, this study's findings support research about inclusive and accessible exhibition development in the following areas: the contribution of an interdisciplinary and participatory approach to exhibit development that can help minimize barriers to access caused by ocularcentric narratives; and the exploration of how non-ocularcentric perspectives can generate new and inclusive narratives for museum exhibitions. This study also contributes to research that explores non-ocularcentric methods for participatory design. And finally, the findings also support research outside disability related subjects by suggesting that distinct sensory abilities and object interpretations because of those abilities, can better support the practice of sensory museology. 


\section{Conclusion}

This research aimed to improve access to museum exhibitions for visitors with vision loss through inclusive narratives. The findings from this study provide a step forward in research focused on accessible exhibition development as well as research in both multisensory and social inclusion in material culture studies. A key factor in my study was the participation of people with non-visual mental models resulting from congenital and early-onset blindness.

My study led to findings to support the development of the six distinctive inclusive themes/exhibition topics grounded in the narratives of participants who are blind: THE VICTORIA ERA AESTHETIC; SPATIAL PRESENCE; TOOLS AND IDENTITY; HACKING; THE SPATIAL PRESENCE OF INFORMATION; and MUSCLE MEMORY. The categories in the thematic analysis, such as community, identity, and sensory abilities amongst others that led to those themes/exhibition topics could be reinterpreted again to develop additional inclusive narratives. My findings also supported the development of a proposed exhibit module that explored these findings and led to its comparison to an existing ocularcentric exhibit. Factors related to the design of remote object reading sessions and working with people who are blind were also findings that emerged from this study.

This work contributes to the existing literature in both inclusion and accessibility in museum exhibitions. It supports prior studies that suggest that accessibility should be considered early in the exhibition development process (Cachia, 2019; Hesseldahl et al., 2018; Osborne et al., 2014) to minimize the 'othering' in visitor experience (ShimamuraWillcocks, 2011) and that people with vision loss can benefit from exhibition messages interpreted using their sensory abilities as a starting point (Chick, 2019; Laurentino et al., 2021). The study's findings have the possibility to add a new dimension to accessible 
museum exhibition development research that enhances curatorial research and exhibition practices that are already embracing participatory and inclusive values.

This work also expands on previous literature in material culture studies and sensory museology. The study's findings point to a possibility of a contribution to material culture studies whereby richer and more diverse narratives may emerge from the thematic analysis of the data derived from people's voices and which may perhaps challenge an object's dominant narrative. The research approach and subsequent findings that emerged echo prior studies that explored the use of cultural probes to gain personal insights (Woodward, 2016), multisensory and non-expert access to embrace social inclusion (Krmpotich, 2020; Peers \& Krmpotich, 2014), and non-ocularcentric practices in sensory museology (Howes, 2014; Mills, 2018). In my study, the findings demonstrate how including people with congenital and early-onset blindness and expanding the multisensory practices to include people with distinct sensory abilities and experiences (Kupers \& Ptito, 2014) can lead to alternative narratives.

A progression in this research would be to explore how participant's age, premature access to the research kit, and previous experience as product testers could have had an impact on the findings. There is also room for future research that can explore improving this study's methodology by using different objects and reshaping the research design to explore or minimize the effects of priming. Other, future research could evaluate visitor experience using a physical prototype of the proposed exhibit module.

Despite the limitations and exploratory nature of my study, it points to the possibility for a new strategy to improve access to inclusive narratives by moving the complexity of accessible museum exhibitions from the visitor with vision loss to the researchers at the outset of the exhibition development process. Overall, this study supports the strategy of interdisciplinary practices, qualitative methods, and 
collaboration with people with congenital and early-onset blindness in exhibit development. Broadly, this study contributes to existing knowledge that values nonocularcentric perspectives and experiences in museum research with the vision that nonvisual practices may someday add a different dimension to an institution's ocularcentric values. This transformation would be in-step with other recent changes in museum research practices that involve source communities, sensory museology, and non-visual representation of historical data (Howes et al., 2018; Krmpotich, 2020; Wood, 2019). Taken together, this study suggests a step forward toward inclusive narratives that could hopefully provide equal access to museum services, nurture the design of accessible exhibitions, and deliver positive visitor experiences by all members of society. 


\section{References}

Abrams, K. M., Wang, Z., Song, Y. J., \& Galindo-Gonzalez, S. (2015). Data Richness Trade-Offs Between Face-to-Face, Online Audiovisual, and Online Text-Only Focus Groups. Social Science Computer Review, 33(1), 80-96. https://doi.org/10.1177/o894439313519733

Abt, J. (2007). The Origins of the Public Museum. A Companion to Museum Studies, 115-134. https://doi.org/10.1002/9780470996836.ch8

Aehnelt, M., Peter, C., \& Müsebeck, P. (2012). A discussion of using mental models in assistive environments. ACM International Conference Proceeding Series. https://doi.org/10.1145/2413097.2413145

Archibald, M. M., Ambagtsheer, R. C., Casey, M. G., \& Lawless, M. (2019). Using Zoom Videoconferencing for Qualitative Data Collection: Perceptions and Experiences of Researchers and Participants. International Journal of Qualitative Methods, 18, 1-8. https://doi.org/10.1177/1609406919874596

Asakawa, S., Guerreiro, J., Ahmetovic, D., Kitani, K. M., \& Asakawa, C. (2018). The present and future of museum accessibility for people with visual impairments. ASSETS 2018 - Proceedings of the 2Oth International ACM SIGACCESS Conference on Computers and Accessibility, 382-384. https://doi.org/10.1145/3234695.3240997

Bennet, T. (1995). The birth of the museum. Routledge.

Bulk, L. Y., Smith, A., Nimmon, L., \& Jarus, T. (2020). A closer look at opportunities for blind adults: Impacts of stigmatization and ocularcentrism. British Journal of Visual Impairment, 38(3), 270-283. https://doi.org/10.1177/0264619620911424

Cachia, A. (2013). Talking Blind: Disability, Access, and the Discursive Turn. Disability Studies Quarterly, 33(3). https://doi.org/10.18061/dsq.v33i3.3758

Cachia, A. (2019). The politics of creative access: Guidelines for a critical dis/ability curatorial practice. In A. Cachia, R. Garland-Thomson, K. Ellis, M. Kent, \& R. Robertson (Eds.), Interdisciplinary Approaches to Disability (pp. 99-108). Routledge. 
Canadian Architect. (2004). Cool '6os Design exhibition to open at the Canadian Museum of Civilization. Canadian Architect. www.canadianarchitect.com/cool-6osdesign-exhibition-to-open-at-the-canadian-museum-of-civilization/

Canadian Museum of History. (2020). Framework for Indigenous Relations.

Candlin, F. (2004). Don't Touch! Hands Off! Art, Blindness and the Conservation of Expertise. Body \& Society, 1O(1), 71-90.

https://doi.org/10.1177/1357034x04041761

Candlin, F. (2008a). Museums, Modernity and the Class Politics of Touching Objects. In H. J. Chatterjee (Ed.), Touch in Museums (pp. 9-20). Berg.

Candlin, F. (2008b). Touch, and the Limits of the Rational Museum or Can Matter Think? The Senses and Society, 3(3), 277-292. https://doi.org/10.2752/174589308x331323

Carmen, P. (2013). A New Model for Access in the Museum. Disability Studies Quarterly, 33(3). https://dsq-sds.org/article/view/3757

Catteneo, Z., \& Vecchi, T. (2011). Blind Vision: The Neuroscience of Visual Impairment. The MIT Press.

Chick, A. (2018). Improving Intellectual Access in Temporary Exhibitions for Sight Loss Visitors Through Co-creation and Co-assessment. Design Journal, 21(4), 561-582. https://doi.org/10.1080/14606925.2018.1472441

Chick, A. (2019). Improving intellectual access for blind and partially sighted visitors to temporary exhibitions: An inclusive design solution. International Journal of the Inclusive Museum, 12(1), 39-62. https://doi.org/10.18848/18352014/CGP/v12io1/39-62

Classen, C., \& Howes, D. (2006). The Museum as Sensescape: Western Sensibilities and Indigenous Artifacts. In E. Edwards, C. Gosden, \& R. Phillips (Eds.), Sensible Objects: Colonialism, Museums and Material Culture (pp. 199-222). Taylor \& Francis.

Collier, A. (2011). The Modern Eye: Craft and Design in Canada 1940-1980. Art Gallery of Greater Victoria. 
Committee on the Rights of Persons with Disabilities. (2014). Convention on the Rights of Persons with Disabilities. United Nations. www.ohchr.org/EN/HRBodies/CRPD/Pages/GC.aspx

Cybenko, G., \& Brewingtont, B. (1999). The Foundations of Information Push and Pull. In The Mathematics of Information Coding, Extraction and Distribution (pp. 930). https://doi.org/DOI:10.1007/978-1-4612-1524-0_2

dominionmodern. (2013). DOMINIONMODERN| The Modern Eye - Craft and Design in Canada, 1940-1980. Dominionmodern.

Eardley, A. F., Mineiro, C., Neves, J., \& Ride, P. (2016). Redefining Access: Embracing multimodality, memorability and shared experience in Museums. Curator: The Museum Journal, 59(3), 263-286.

Employment and Social Development Canada. (2021). C-81_4. Making an Accessible Canada for Persons with Disabilities. https://www.canada.ca/en/employmentsocial-development/programs/accessible-canada.html

Everrett, T. (2019). A Curatorial Guide to Museum Sound Design. Curator, 62(3), 313-325. https://doi.org/10.1111/cura.12305

Falk, J. H. (2009). Identity and the Museum Visitor Experience. Left Coast Press.

Fogle-Hatch, C. (2021). MuseumSenses.

French, J. (2019). Auto Agents: Inclusive curatorship and its political potential. In R. R. Janes \& R. Sandell (Eds.), Museum Activism (pp. 152-163). Routledge. https://doi.org/10.4324/9781351251044

Garland-Thomson, R. (2019). Critical disability studies: a knowledge manifesto. In M. Kent, K. Ellis, R. Robertson, \& R. G. Thomson (Eds.), Manifestos for the Future of Critical Disability Studies (Vol. 1, pp. 11-19). Routledge. https://doi.org/10.4324/9781351053341

Gaver, B., Dunne, T., \& Pacenti, E. (1999). Cultural Probes. Interactions, 6(1), 21-29. https://doi.org/10.1515/9783110677515-015

Hadlaw, J. (2019). Design Nationalism, Technological Pragmatism and the Performance of Canadian-ness: The Case of the Contempra Telephone. Journal of Design History, 32(3), 240-262. https://doi.org/10.1093/jdh/epzo13 
Handa, K., Dairoku, H., \& Toriyama, Y. (2010). Investigation of priority needs in terms of museum service accessibility for visually impaired visitors. The British Journal of Visual Impairment, 28(3), 221-234. https://doi.org/10.1177/0264619610374680

Harrison, J. D. (1994). Ideas of Museums In the 1990s. Museum Management and Curatorship, 13(2), 176. https://doi.org/10.1080/o9647779409515396

Hesseldahl, K., McGinley, C., \& Monk, G. (2018). Using design thinking to develop new methods of inclusive exhibition making. In G. Craddock, C. Doran, L. McNutt, \& R. Dónal (Eds.), Studies in Health Technology and Informatics (Vol. 256, pp. 151-160). IOS Press. https://doi.org/10.3233/978-1-61499-923-2-151

Hirose, K. (2013). Research on Methods of "Touching the World" - The Aim of the Exhibit Area of Tactile Learning in Japan's National Museum of Ethnology. Disability Studies Quarterly, 33(3). https://doi.org/10.18061/dsq.v33i3.3743

Holmes, K. (2018). Mismatch: how inclusion shapes design. The MIT Press.

Howes, D. (2014). Introduction to sensory museology. Senses and Society, 9(3), 259-267. https://doi.org/10.2752/174589314X14023847039917

Howes, D., Clarke, E., Macpherson, F., Best, B., \& Cox, R. (2018). Sensing art and artifacts: explorations in sensory museology. Senses and Society, 13(3), 317-334. https://doi.org/10.1080/17458927.2018.1516024

Hughes, P. (2015). Exhibition design (2nd ed.). Laurence King Publishing.

Hunchuck, S. H., Design, C., Alfody, S., Bourassa, P., Cordner, B., Prokopow, M., \& Elder, A. C. (2006). Compte rendu d'exposition. Material Culture Review, 63(Spring), 80-88.

Hunzinker, L. (2019). After the Wall, Juicy Salif exhibit. Vitra Design Museum.

Ingenium. (2018). INGENIUM ACCESSIBILITY.

International Council of Museums. (2007). Museum Definition. https://icom.museum/en/resources/standards-guidelines/museum-definition/ Johnson, K. (2001). Planning and Designing Exhibition Facilities. In B. Lord \& G. Lord (Eds.), The Manual of Museum Exhibitions (pp. 69-142). AltaMira Press.

Krmpotich, C. (2020). The senses in museums. In R. Skeates \& J. Day (Eds.), The Routledge Handbook of Sensory Archaelogy (1st ed., pp. 94-106). Routledge. 
Kudlick, C., \& Luby, E. M. (2019). Access as activism. In R. R. Janes \& R. Sandell (Eds.), Museum Activism (1st ed., p. 436). Routledge. https://doi.org/https://doiorg.proxy.library.carleton.ca/10.4324/9781351251044

Kupers, R., \& Ptito, M. (2014). Compensatory plasticity and cross-modal reorganization following early visual deprivation. Neuroscience and Biobehavioral Reviews, 41, 36-52. https://doi.org/10.1016/j.neubiorev.2013.08.001

Laurentino, T., Xavier, M., Ronco, F., Pina-Martins, F., Domingues, I., Penha, B., Dias, M., de Sousa, A., Carrilho, T., Rodrigues, L., Pinheiro, C., Rato, D., Balata, D., Ayala-Botto, G., Matos, M., Campelo, M., \& Botelho, R. (2021). evALLution: making basic evolution concepts accessible to people with visual impairments through a multisensory tree of life. Evolution Education \& Outreach, 14(4), 14. https://doi.org/DOI: 10.1186/s12052-021-00143-1

Levent, N., \& Pascual-Leone, A. (2014). The Multisensry Museum (N. Levent \& A. Pascual-Leone (eds.)). Rowan \& Littlefield Publishers.

Löf, O. (2020). 1989 - Culture and Politics exhibition. Utställningskritik. https://utstallningskritik.se/2020-1/reflektion-over-1989-kultur-och-politik-panationalmuseum/

Lorenc, J., Skolnick, L., \& Berger, C. (2007). What is Exhibition Design. RotoVision. Maberley, D. A. L., Hollands, H., Chuo, J., Tam, G., Konkal, J., Roesch, M., Veselinovic, A., Witzigmann, M., \& Bassett, K. (2006). The prevalence of low vision and blindness in Canada. Eye, 2o(3), 341-346. https://doi.org/10.1038/sj.eye.6701879

Martin, D. (2007). Joybubbles, 58, Peter Pan Of Phone Hackers, Dies. New York Times, B7.

Mashburn, W. C. (2019). Meaningful Inclusion. International Contemporary Art, 11.

Mason, J. (2018). Handing Heritage Objects. Canadian Conservation Institute. https://www.canada.ca/en/conservation-institute/services/preventiveconservation/guidelines-collections/handling-heritage-objects.html

McCulloh, D. (2013). Blind Photographers: Vision, Accessibility, and Empowerment in the Museum. Disability Studies Quarterly, 33(3), 1. https://dsqsds.org/issue/view/104 
Mills, A. (2018). Engaging aesthetically with tapa barkcloth in the museum. Senses and Society, 13(3), 367-374. https://doi.org/10.1080/17458927.2018.1516025

Moreno, L. A., \& Villalba, E. R. (2018). Transdisciplinary Design: Tamed complexity through new collaboration. Strategic Design Research Journal, 11(1), 42-50. https://doi.org/10.4013/sdrj.2018.111.07

Morphy, H. (2015). The Displaced Local. In A. Witcomb \& K. Message (Eds.), The International Handbooks of Museum Studies (pp. 365-387).

Murray, S. (2013). CMHR to Feature the Most Inclusive Design in Canadian History. Marketwire, 1-5.

Nationalmuseum. (2021). 1989 - Culture and Politics. Nationalmuseum. www.nationalmuseum.se/en/1989-kultur-och-politik

Norman, D. A. (2004). Emotional Design: Why We Love (or Hate) Everyday Things. Basic Books. https://doi.org/10.1089/g4h.2015.0070

Norman, D. A. (2010). Living with Complexity. MIT Press.

Osborne, F., Luby, E. M., \& Fogarty, C. (2014). An Accessibility Adventure: Teaching and Learning Exhibition Design Through the Lens of Disability Studies. Exhibitionist, January, 59-63.

Papadimitriou, N., Plati, M., Markou, E., \& Catapoti, D. (2016). Identifying Accessibility Barriers in Heritage Museums: Conceptual Challenges in a Period of Change. Museum International, 68(3-4), 33-47. https://doi.org/10.1111/muse.12134

Parr, J. (1999). Domestic Goods: The Material, the Moral, and the Economic in the Postwar Years (review). In Domestic Goods: The Material, the Moral, and the Economic in the Postwar Years (Vol. 42, Issue 3). https://doi.org/10.1353/tech.2001.0097

Partington-Sollinger, Z., \& Morgan, A. (2011). Shifting Perspectives: Opening Up Museums and Galleries to Blind and Partially Sighted People. In CulturelLnk (p. 88). RNIB.

Pearce, S. M. (1994). Museum Objects. In S. M. Pearce (Ed.), Interpreting Objects and Collections (pp. 9-11). Routledge. 
Peers, L. L., \& Krmpotich, C. (2014). This is our life: Haida material heritage and changing museum practice. In Choice Reviews Online (Vol. 51, Issue 08). UBC Press. https://doi.org/10.5860/choice.51-4527

Perschon, M. (2012). The steampunk aesthetic: Technofantasies in a neo-victorian retrofuture. Univesity of Alberta.

Pesant, M. (2016). DX Uncrated: Classic Plastics exhibit detail. Megan Pesant Photographer. www.meganpesant.com/recentwork/2016/2/24/dx-uncratedclassic-plastics-members-night

Prown, J. D. (1994). Mind in matter: an introduction to material culture theory and method. In S. M. Pearce (Ed.), Interpreting Objects and Collections (pp. 133-138).

Puxley, C., Canadian, T., \& Feb, T. T. (2016, February 16). Blind photographers hope exhibit of work will expose talent, shatter stereotypes. The Canadian Press, 1-3.

Reid, D. J., \& Reid, F. J. M. (2005). Online Focus Groups. International Journal of Market Research, 47(2), 131-164. https://doi.org/10.1080/o0913367.2016.1252288

Reyes-Cruz, G., Fischer, J. E., \& Reeves, S. (2020). Reframing Disability as Competency: Unpacking Everyday Technology Practices of People with Visual Impairments. CHI 2O2O, 1-13. https://doi.org/10.1145/3313831.3376767

Rieger, J., Herssens, J., \& Strickfaden, M. (2020). Spatialising differently through ability and techné. CoDesign, 16(2), 135-151. https://doi.org/10.1080/15710882.2018.1531134

Rieger, J., \& Strickfaden, M. (2016). Taken for Granted: Material Relations Between Disability and Codes/Guidelines. Societies, 6(1), 6. https://doi.org/10.3390/soc6010006

Robson, C., \& McCartan, K. (2016). Real World Research (4th ed.). Wiley.

Saldaña, J. (2016). The Coding Manual for Qualitative Researchers (J. Seaman (ed.); 3rd ed.). Sage Publishing Ltd.

Sanders, E. B. N., \& Stappers, P. J. (2007). Co-creation and the new landscapes of design. CoDesign, 4(1), 5-18. https://doi.org/10.1080/15710880701875068 
Sanders, E. B. N., \& Stappers, P. J. (2014). Probes, toolkits and prototypes: Three approaches to making in codesigning. CoDesign, $10(1), 5^{-14}$. https://doi.org/10.1080/15710882.2014.888183

Saunders, J. (2014). Conservation in Museums and Inclusion of the Non-Professional. Journal of Conservation and Museum Studies, 12(1), 6. https://doi.org/10.5334/jcms.1021215

Schlereth, T. J. (1986). Material Culture or Material Life. In G. L. Pocius (Ed.), Living in a Material World (pp. 231-240). ISER.

Shah, P. K. (2016). Retinopathy of prematurity: Past, present and future. World Journal of Clinical Pediatrics, 5(1), 35. https://doi.org/10.5409/wjcp.v5.i1.35

Sharples, S., \& Cobb, S. (2015). Methods of Collectin and Observing Participant Responses. In J. R. Wilson \& S. Sharples (Eds.), Evaluation of Human Work (4th ed., pp. 83-118). CRC Press.

Shelton, A. A. (2007). Museums and Anthropologies: Practices and Narratives. In S. Macdonald (Ed.), A Companion to Museum Studies (pp. 64-80). Blackwell Publishing.

Shimamura-Willcocks, Y. (2011). Social Inclusion and Museums: Understanding 'Self' and 'Other' in the Context of Japanese Society and Visually Impaired People. University of Leicester.

Silva, C. F., \& Howe, P. D. (2012). The (In)validity of Supercrip Representation of Paralympian Athletes. Journal of Sport and Social Issues, 36(2), 174-194. https://doi.org/10.1177/0193723511433865

Smith, K. (2013). Digital outcasts: moving technology forward without leaving people behind. Morgan Kaufmann.

Smith, M. (2007). Producing Sense, Consuming Sense, Making Sense: Perils and Prospects for Sensory History. Journal of Social History, 4O(4), 841-858. https://doi.org/134.117.10.200

Smithsonian. (2012). Smithsonian Guidelines for Accessible Exhibition Design.

Stemmer, B. (2019). No Title. France Télévisions.

Stolnitz, J. (1993). The Aesthetic Attitude. In P. Alperson (Ed.), The Philosphy of the Visual Arts (1st ed., pp. 7-14). Oxford University Press. 
The National Museum Stockholm. (2019). 1989-Culture and Politics exhibit detail. (Smow).

Topping, M., Douglas, J., \& Winkler, D. (2021). General Considerations for Conducting Online Qualitative Research and Practice Implications for Interviewing People with Acquired Brain Injury. International Journal of Qualitative Methods, 2O, 1-15. https://doi.org/10.1177/16094069211019615

Utah Arts Alliance. (2021). dreamscapes. https://www.dreamscapesslc.org

Vaz, R., Freitas, D., \& Coelho, A. (2020). Blind and visually impaired visitors' experiences in museums: Increasing accessibility through assistive technologies. International Journal of the Inclusive Museum, 13(2), 57-80. https://doi.org/10.18848/1835-2014/CGP/V13Io2/57-80

Vitra Schaudepot. (2020). After the Wall: Design since 1989. Vitra Design Museum. vom Lehn, D. (2010). Discovering "Experience-ables": Socially including visually impaired people in art museums. Journal of Marketing Management, 26(7-8), 749-769. https://doi.org/10.1080/02672571003780155

Wapner, J. (2013). Mission and Low Vision: A Visually Impaired Museologist's Perspective on Inclusivity. Disability Studies Quarterly, 33(3), 1.

Wobbrock, J. O., Kane, S. K., Gajos, K. Z., Harada, S., \& Froehlich, J. (2011). Abilitybased design: Concept, principles and examples. ACM Transactions on Accessible Computing, 3(3), 1-27. https://doi.org/10.1145/1952383.1952384

Wood, C. M. (2019). Songs of the Ottawa. Carleton University.

Woodward, S. (2016). Object interviews, material imaginings and 'unsettling' methods: interdisciplinary approaches to understanding materials and material culture. Qualitative Research, 16(4), 359-374. https://doi.org/10.1177/1468794115589647 


\title{
8 Appendices
}

\section{APPENDIX A CUREB CLEARANCE}

\author{
Carleton \\ Canada's Capital University
}

Office of Research Ethics

4500 ARISE Building | 1125 Colonel By Drive

Ottawa, Ontario K1S 5B6

613-520-2600 Ext: 4085

ethics@carleton.ca

CERTIFICATION OF INSTITUTIONAL ETHICS CLEARANCE

The following research has been granted clearance by the Carleton University Research Ethics Board-B (CUREB-B). CUREB-B is constituted and operates in compliance with the Tri-Council Policy Statement: Ethical Conduct for Research Involving Humans (TCPS2).

Ethics Clearance ID: Project \# 114508

Project Team Members: Ms. Carla Ayukawa (Primary Investigator)

Tom Everrett (Research Supervisor)

Chantal Trudel (Research Supervisor)

Study Title: Accessible museum exhibitions: interpreting museum objects using distinctive sensory competencies

Funding Source: (If applicable):

Effective: October 16, 2020

Expires: October 31, 2021

This certification is subject to the following conditions:

1. Clearance is granted only for the research and purposes described in the application.

2. Any modification to the approved research must be submitted to CUREB-B via a Change to Protocol Form. All changes must be cleared prior to the continuance of the research.

3. An Annual Status Report for the renewal or closure of ethics clearance must be submitted and cleared by the renewal date listed above. Failure to submit the Annual Status Report will result in the closure of the file. If funding is associated, funds will be frozen. 


\title{
APPENDIX B RECRUITMENT POSTER
}

\author{
Carleton \\ U N I VER S I T Y \\ Canada's Capital University
}

\section{Would you like to improve accessibility in museum exhibitions?}

We invite you to participate in a study on the development of accessible museum exhibitions using distinctive sensory abilities.

To participate, you must be the following:

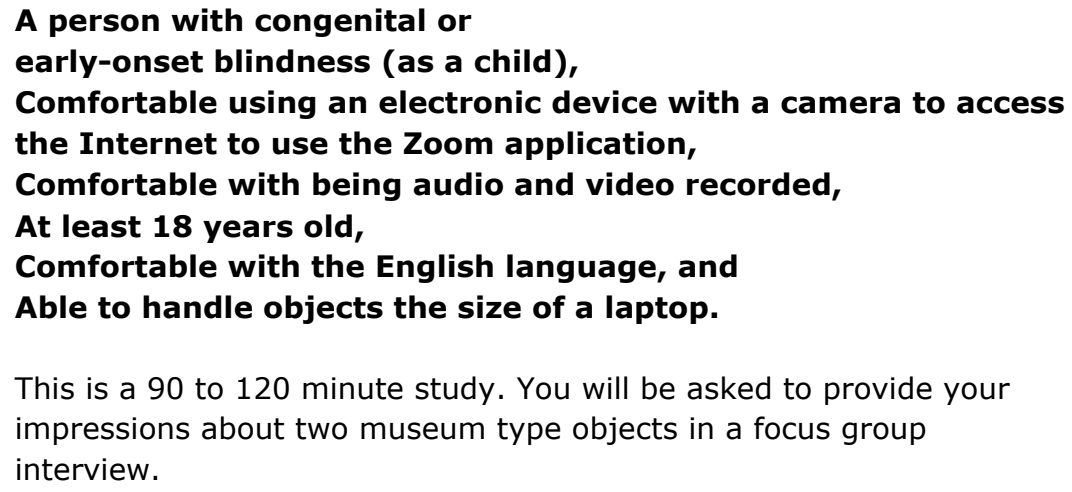

This is a 90 to 120 minute study. You will be asked to provide your impressions about two museum type objects in a focus group interview.

The study will be recorded through audio and video online over Zoom from your home or wherever you feel comfortable.

Participants will be compensated with a $\$ 100$ honorarium.

This study has been cleared by the Carleton University Research Ethics Board B (Clearance \#114508).

Please contact the researcher, Carla Ayukawa, for more details on this study at carlaayukawa@cunet.carleton.ca.

This study is supported by: Ingenium, Canada's Museums of Science and Innovation and the Canadian Council of the Blind. 


\section{APPENDIX C INTERVIEW SCRIPT}

- Do not say "phone"

- Refer to Object 2 as "Telephone"

- Refer to the video as "Camera"

\section{Set up Zoom}

- Introductions

- Did anyone have trouble with the Zoom link?

- Video Camera

- Audio and Video record check

- If for some reason Zoom fails, please just log back in

- Questions

- I'll start the video recording

\section{Review contents of box}

- Open up research kit if you haven't already - braille label to indicate which side to open

- Inside there are 2 more boxes and 3 envelopes

- Boxes labeled Object 1 and Object 2

- Envelopes labeled Consent form, Nitrile Gloves, Return Label

- We are using Nitrile Gloves because that is what curators use. There are two sizes.

- Questions?

\section{Review session}

- The research is NOT to test you.

- I want this to be a discussion between us all about the objects. So I encourage you to think aloud and build off each other's comments.

- We will be examining one object at a time together

- I'll be giving you some directions.

- At the end we will take off the gloves

- We will pack up the boxes and label

- It will take around $1 \frac{1 / 2}{2}$ to 2 hours - we can go longer if you like

- If at anytime you need a break, please tell me. We will also be taking a break between objects.

- Questions? 


\section{Session}

Script notes:

- Don't worry about what the object is right now

- I really want you to be able to give me your own impressions about it so we can discuss it.

- Please feel free to pick it up, turn it around, move it if it has moving parts.

- If you can think aloud while you do that, it will help with the discussion.

\section{Sensory Descriptions}

a. What are your first impressions about this object? How does it feel in your hands?

i. Let's start with its weight.

ii. ...shape

iii. ...surface textures

iv. ...materials

b. What do you consider is the most interesting thing about this object?

Script notes:

- Now that you have had a chance to get to know the object.

c. Does its weight, shape, surface sound... remind you of a memory or a moment in your life? That you would associate with the telephone?

d. How would you describe this object to someone with low vision? Is there something that it is similar that you could compare it to?

Script notes: Introducing its function here

- It is a (telephone, fruit juicer)

- The telephone is from the 1960-70's. It could hang on the wall or on a table and there was even a hang-up button on the handset.

- The juicer is from the 1990's. A glass is placed between the legs and then half a lemon or orange is placed onto and then when it is squeezed against the rounded part, the juice would drip into the glass.

\section{Insights about its function and design}

a. How do you think it weight shape sound contribute to its function?

b. Is there anything about its design that you really like?

c. What is would be your personal rating of its design? (1 for low and 10 for high)

d. What do you base your rating on?

e. Why is that important to you?

f. Describe do you think would own something like this?

Script notes: Introducing its dominant narrative here (include the script)

- These objects have been included in museum exhibitions. The story that usually goes with the objects is:

- Juicy Salif - represents a design that is part art, part functional - though it is well known for not being able to work well as a juicer. But it was successful 
because of its sculptural shape. People bought it because it was different and not because they needed a juicer. It is considered an Emotional Design.

- Contempra telephone - represents post-war (WW2), modernist Canadian design. This is because of its basic shape, uses plastic as itself and not trying to be a reproduction of wood and is manufactured very efficiently and fast. It also was marketed as something contemporary for its time and was offered in different colours. That was a big selling point for when the government wanted to stimulate the economy and get people buying after the depression.

\section{Other narratives - to reexamine}

a. How could this object be associated with a memory from your own life?

b. Are there any memories that are triggered by something about this object?

c. Is there something about this object that you think would be of interest to someone who is blind or low vision?

Script notes: Nitrile gloves and sensory experience

- If you feel comfortable please remove your gloves to touch the objects.

\section{Nitrile gloves and sensory experience}

a. Is there anything about the object that seems different?

\section{Reflection}

a. Can you describe something from this exercise that was really interesting to you?

\section{End notes}

- Anything you would like to mention?

- Honorarium - cheque, etransfer?

\section{Re-packaging}

- The packing label is in the box

- Remove old label

- Use the packing tape supplied to close the box

- Apply label to the top of the box

\section{Return Shipping}

1. I will call FedEx to pick up 1-800-463-3339 to schedule

2. Account \# 


\section{APPENDIX D ROUND-TABLE DISCUSSION}

1) Purpose of discussion.

a. The goal of the discussion is to hear your comments on the proposed inclusivity and accessibility of an exhibit topic and design and if it might create an environment where visitors can have a share museum experience.

2) Background of events leading up to round table discussion.

a. From your comments in the research sessions, emerged several exhibit themes.

b. From the different exhibit themes, one was developed into an exhibit scenario.

c. This exhibit is about Muscle Memory and uses the Contempra telephone to convey the message.

3) The exhibit's Big Idea and its extended messages.

a. The Big Idea communicates how muscle memory plays a role in our everyday lives by creating maximum efficiency within our system to complete a task.

b. The exhibit explains what muscle memory is: It is a form of memory that is created through the repetition of a specific motor task - such as dialing a telephone number. The process decreases the need for attention and creates maximum efficiency within motor and memory systems.

c. Through tactile and audio-based interactives, the exhibit provides shared learning experiences of how muscle memory impacts our everyday lives such as sports, music, travel routes home from work or school, tying your shoes, etc.

4) How the Contempra telephone fits in?

a. The Contempra telephone demonstrates the relationship between the task and our muscles that do the task. Our body remembers how to execute the task based on how our muscles are trained. Using the telephone as an example was inspired from our research sessions.

b. The Contempra telephone also demonstrates that how its design incorporated touchtone dialing technology and, as I learned from you, how touchtone was more desirable as Contempra transitioned from rotary to touchpad.

5) Encountering the exhibit as a visitor.

a. The following is an exhibit visitor experience scenario that uses some of your comments as inspiration.

b. Imagine walking into a museum with a group of friends or with family. You are beckoned toward an exhibit (via a proximity sensor) by an audio message. This audio message invites you to visit the Muscle Memory exhibit. The message encourages you "to learn how muscle memory plays a role in your everyday life as you interact with the objects around your and the tasks you do".

c. As you approach the exhibit, you can hear the sound of a traditional telephone ring and a message inviting you to "Answer the telephone". When you lift the handset you hear a voice telling you about the exhibit that you are 
about to enter. This voice is also heard through a directed speaker for others to hear.

d. The 'voice' gives you some instructions about how to interact with the exhibit. It tells you that there are a series of handsets to be lifted to hear more about the topic.

e. Each subsequent handset has a different method of 'dailing'. The audio from the handset tells you the relationship between your muscles, your memory, and the repetitive tasks you do everyday.

f. You are free to lift any of the handsets in any order to learn different things about the exhibit topic. You would be able to select what interests you by pressing a button on the keypad much like when you call a bank.

g. Interactive activities for the exhibit will challenge your different muscle memory skills as part of active learning. One activity would ask you to dial the same telephone number into different types of dialing interfaces - rotary, touchtone, and computer keyboard. The sound from the different dials could be important too. You could also be challenged on how fast you would be able to input a number without making a mistake. The challenge could be reminiscent of dialing into a radio station contest - you could compete against for friends or other visitors.

h. The audio you hear from the handset also acts as object labels and provides you with an integrated audio description about the Contempra telephone. It describes how the telephone was designed and produced at a time when the input interfaces were changing as a result of changing technology.

i. The audio description of the Contempra telephone describes it as:

"The Contempra telephone is an example of dials evolving from analog rotary to digital touchpad and requiring a new set of memory tools to input numbers."

"Its long and streamlined form, about the size of a paperback book was notably heavier compared to today's telephones. A shiny yet featureless exterior made it appear restful on the tables or walls of many family homes and offices."

6) The entire exhibit would be audio and tactile based.

a. The exhibit uses audio to deliver the content. The audio would come from the handset and could simultaneously be heard through a directional speaker allowing your entire group to hear.

b. The tactility of the handsets and the subsequent variety of dialing interfaces would be as accurate as possible to reinforce the relationship between muscles, repetitive tasks, and memory.

c. The handset, with its angled shape, would allow you to hold it 'hands-free' on your shoulder.

d. The audio feedback from the handsets in the form of clicks from the rotary dial or tones from the touch pad are also used to reinforce the message of memory and repetitive tasks - visitors would be asked to input numbers on a 
touchtone phone and hear a tune - they could then be asked to reproduce that tune by dialing on the pad again - but this time from memory.

Suggestions will be given on how to remember numbers using a touch pad by focusing on the 'shape' of the number or the movement of your fingers.

e. Deep-dives into the exhibit content could talk about phone numbers that have been burned into your memory like that of your best friend, 911 emergency, or telephone numbers used as a advertising device.

f. You would be invited to share a memorable number and/or story about it by recording yourself through a telephone connected to an 'answering machine'. Your recording would be saved for other visitors to hear and as an audio artifact for the museum collection.

g. The design of the exhibit structure offers a surface rail and act as a wayfinding devise allowing you independently explore the exhibit. The surface texture would transition depending on the section you are in or indicate the presence of an object. 